\title{
An Ejector Air Intake Design Method for a Novel Rocket-Based Combined-Cycle Rocket Nozzle
}

\author{
by \\ Timothy S. Waung \\ B.A.Sc.
}

\begin{abstract}
A thesis submitted to
the Faculty of Graduate Studies and Research

in partial fulfillment of

the requirements for the degree of

Master of Applied Science
\end{abstract}

Ottawa-Carleton Institute for

Mechanical and Aerospace Engineering

Department of Mechanical and Aerospace Engineering

Carleton University

Ottawa, Ontario, Canada

April 2010

(C) Timothy S. Waung 2010

All rights reserved. 


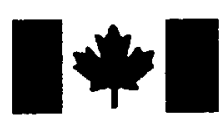

\author{
Library and Archives \\ Canada \\ Published Heritage \\ Branch \\ 395 Wellington Street \\ Ottawa ON K1A ON4 \\ Canada
}

Bibliothèque et

Archives Canada

Direction du

Patrimoine de l'édition

395 , rue Wellington

Ottawa ON K1A ON4

Canada
Your file Votre référence
ISBN: 978-0-494-68649-2
Our file Notre reférence
ISBN: $978-0-494-68649-2$
NOTICE:

The author has granted a nonexclusive license allowing Library and Archives Canada to reproduce, publish, archive, preserve, conserve, communicate to the public by telecommunication or on the Internet, loan, distribute and sell theses worldwide, for commercial or noncommercial purposes, in microform, paper, electronic and/or any other formats.

The author retains copyright ownership and moral rights in this thesis. Neither the thesis nor substantial extracts from it may be printed or otherwise reproduced without the author's permission.
AVIS:

L'auteur a accordé une licence non exclusive permettant à la Bibliothèque et Archives Canada de reproduire, publier, archiver, sauvegarder, conserver, transmettre au public par télécommunication ou par l'Internet, prêter, distribuer et vendre des thèses partout dans le monde, à des fins commerciales ou autres, sur support microforme, papier, électronique et/ou autres formats.

L'auteur conserve la propriété du droit d'auteur et des droits moraux qui protège cette thèse. $\mathrm{Ni}$ la thèse ni des extraits substantiels de celle-ci ne doivent être imprimés ou autrement reproduits sans son autorisation.
In compliance with the Canadian Privacy Act some supporting forms may have been removed from this thesis.

While these forms may be included in the document page count, their removal does not represent any loss of content from the thesis.
Conformément à la loi canadienne sur la protection de la vie privée, quelques formulaires secondaires ont été enlevés de cette thèse.

Bien que ces formulaires aient inclus dans la pagination, il n'y aura aucun contenu manquant.

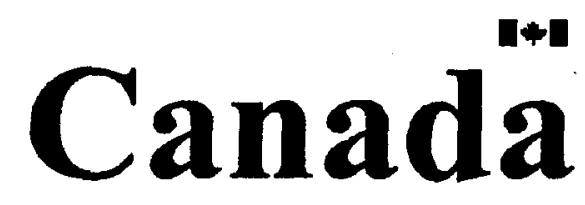


The undersigned recommend to

the Faculty of Graduate Studies and Research

acceptance of the thesis

\section{An Ejector Air Intake Design Method for a Novel Rocket-Based Combined-Cycle Rocket Nozzle}

Submitted by Timothy S. Waung, B.A.Sc.

in partial fulfillment of the requirements for the degree of

Master of Applied Science

J. Etele, Supervisor

M. Yaras, Department Chair

Carleton University

2010 
I hereby declare that I am the sole author of this thesis.

I authorize Carleton University to lend this thesis to other institutions or individuals for the purpose of scholarly research.

I further authorize Carleton University to reproduce this thesis by photocopying or by other means, in total or in part, at the request of other institutions or individuals for the purpose of scholarly research. 


\section{Abstract}

Rocket-based combined-cycle (RBCC) vehicles have the potential to reduce launch costs through the use of several different air breathing engine cycles, which reduce fuel consumption. The rocket-ejector cycle, in which air is entrained into an ejector section by the rocket exhaust, is used at flight speeds below Mach 2. This thesis develops a design method for an air intake geometry around a novel RBCC rocket nozzle design for the rocket-ejector engine cycle. This design method consists of a geometry creation step in which a three-dimensional intake geometry is generated, and a simple flow analysis step which predicts the air intake mass flow rate. The air intake geometry is created using the rocket nozzle geometry and eight primary input parameters. The input parameters are selected to give the user significant control over the air intake shape. The flow analysis step uses an inviscid panel method and an integral boundary layer method to estimate the air mass flow rate through the intake geometry. Intake mass flow rate is used as a performance metric since it directly affects the amount of thrust a rocket-ejector can produce. The design method results for the air intake operating at several different points along the subsonic portion of the Ariane 4 flight profile are found to under predict mass flow rate by up to $8.6 \%$ when compared to three-dimensional computational fluid dynamics simulations for the same air intake. 


\section{Acknowledgments}

First of all, I would like to thank Dr. Etele for giving me the opportunity to work on this project as I have always been interested in fluids and aerospace. Through the process of completing this thesis I have learned so much and I am sure the variety of tasks required to complete this project will be valuable to me in the future. I would like to thank Dr. Etele for providing me valuable guidance as well as addressing all my questions and concerns with patience and humor. I would also like to thank Dr. Etele for allowing me to present a paper at a conference in Denver and for allowing me to stay in a very nice hotel. I would like to thank Dr. Matida for allowing access to his computational cluster in order to run CFD simulations. Thanks to my fellow grad students in ME 3129 for welcoming me into the lab.

Special thanks to my extended family in Ottawa for welcoming me and for helping me get settled here and showing me around town. Finally I would like to thank my parents,

siblings, and grandparents back in Burnaby for their support, updates from home, and especially the care packages filled with food! 


\section{Table of Contents}

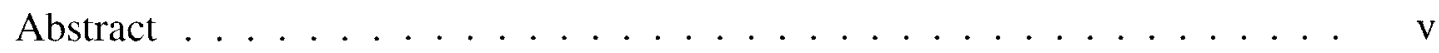

Acknowledgments .............................. vi

Table of Contents .............................. vii

List of Tables ........................ . . .

List of Figures . . . . . . . . . . . . . . . . . xi

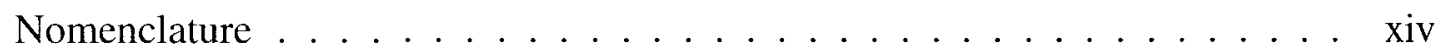

1 Introduction 1

1.1 Rocket-Based Combined-Cycle Engines . . . . . . . . . . . . . . 2

1.2 Novel RBCC Rocket Nozzle . . . . . . . . . . . . . . . . . . . . . . 6

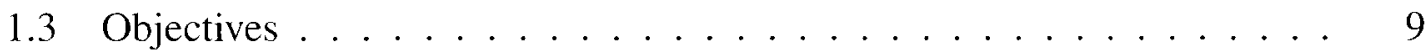

1.4 Intake Analysis Literature Review . . . . . . . . . . . . . . . . 11

1.4.1 Inviscid Flow Solution Methods . . . . . . . . . . . . . . . 12

1.4.2 Viscous Flow Solution Methods . . . . . . . . . . . . . . . . 14

1.4 .3 Inviscid-Viscous Interaction Methods . . . . . . . . . . 15

1.4.4 Full Navier-Stokes Solvers . . . . . . . . . . . . . 18

2 Air Intake Design Method: Geometry Creation 19

2.1 Fairing Geometry . . . . . . . . . . . . . . . 22

2.2 Centre Body Geometry . . . . . . . . . . . . . . . . . 27

2.3 Cowl Geometry . . . . . . . . . . . . . . . . 30

2.4 3D Geometry Creation . . . . . . . . . . . . . . . . . 34

2.5 Geometry Results . . . . . . . . . . . . . . . 35 
3 Air Intake Design Method: Flow Analysis 41

3.1 Flow Condition Input Parameters . . . . . . . . . . . . . . . . . 42

3.2 Inviscid Flow Solver . . . . . . . . . . . . . . . 43

3.2.1 Potential Flow Panel Methods . . . . . . . . . . . . 43

3.2 .2 Combination Procedure .............. 53

3.2.3 Velocity Scaling .................. 59

3.2 .4 Compressibility Correction . . . . . . . . . . . . 61

3.3 Viscous Flow Solver . . . . . . . . . . . . . . 63

3.3.1 Integral Boundary Layer Method . . . . . . . . . . . . 63

3.3.2 Total Pressure Losses . . . . . . . . . . . . . . . . 68

3.4 Air Mass Flow Rate Calculation . . . . . . . . . . . . . . . 70

3.5 Flow Analysis Panel Sensitivity . . . . . . . . . . . . . . 72

3.6 Flow Analysis Validation . . . . . . . . . . . . . . 75

4 Air Intake Design Method Case Study $\quad 80$

4.1 Rocket Nozzle Geometry Selection . . . . . . . . . . . . . . . . . 80

4.2 Flight Profile Selection and Rocket-Ejector Configuration . . . . . . . . 81

4.3 Geometry Selection . . . . . . . . . . . . . . . 84

5 CFD Simulations $\quad 88$

5.1 Discretization Method . . . . . . . . . . . . . . . . . . 89

5.2 Turbulence Model . . . . . . . . . . . . . . . . . . . . 89

5.3 Flow Domain and Boundary Conditions . . . . . . . . . . . . 90

5.4 Grid and Grid Convergence Study . . . . . . . . . . . . . . . . 93

5.5 Convergence History . . . . . . . . . . . . . . . . . . 96

5.6 . CFD Results . . . . . . . . . . . . . . . . . . . . . . . . 98

5.6.1 Pressure Distribution . . . . . . . . . . . . . . . 99

5.6 .2 Boundary Layer ... . . . . . . . . . . . . 101

5.6 .3 Total Pressure Loss . . . . . . . . . . . . . . . . 107

5.6 .4 Mass Flow Rate . . . . . . . . . . . . . . . . . . . 110 
6 Conclusions and Recommendations

113

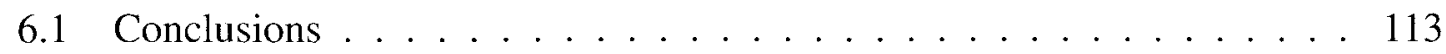

6.2 Recommendations . . . . . . . . . . . . . . 115

References

118 


\section{List of Tables}

2.1 Input Parameter Description $\ldots \ldots \ldots \ldots \ldots$

2.2 Primary Input Parameter Summary . . . . . . . . . . . . . 36

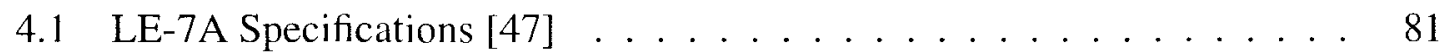

4.2 Flight Profile Breakdown . . . . . . . . . . . . . 81

4.3 Selected Air Intake Geometry $\ldots \ldots \ldots \ldots$

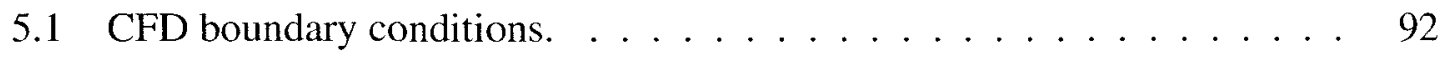

5.2 Grid sizes . . . . . . . . . . . . . . . . . . . . . . . . . 94

5.3 Grid convergence study results $\ldots \ldots \ldots \ldots \ldots \ldots$ 


\section{List of Figures}

1.1 Typical specific impulse performance for various propulsion cycles. Ref [7] 3

1.2 Schematic of an RBCC engine. . . . . . . . . . . . . . . 4

1.3 Proposed rocket exhaust configurations by Escher and Schnurstein. Ref [12] 5

1.4 Three-view of NASA GTX concept vehicle. Ref [5] . . . . . . . . . 6

1.5 Example of novel $\mathrm{RBCC}$ rocket nozzle geometry. . . . . . . . . . . 7

1.6 Section view of nozzle along symmetry plane $2 \ldots \ldots . \ldots 8$

1.7 View of RBCC engine with novel RBCC rocket nozzle. . . . . . . . . 8

1.8 Example of rocket nozzle geometry variation. . . . . . . . . . 8

1.9 Inviscid and viscous flow zones in a diffuser . . . . . . . . . . . . . 16

2.1 Example of novel RBCC air intake geometry . . . . . . . . . . . 20

2.2 Fairing geometry profiles .................. 23

2.3 Fairing construction sequence. . . . . . . . . . . . 25

2.4 Fairing profile definitions. . . . . . . . . . . . . 26

2.5 Cross section view along symmetry plane 1 of proposed air intake geometry. 28

$2.6 \mathrm{x}$-y cross section view of outflow plane. . . . . . . . . . . . . 29

2.7 Relationship between flow area and $r_{C B_{3}} \ldots \ldots \ldots$. . . . . . . . 29

2.8 Cowl lip geometry definitions . . . . . . . . . . . . . . . 32

2.9 Cowl outer and inner blend regions . . . . . . . . . . . . . . . 33

2.10 Top view of 3D geometry, highlighting fillet surfaces. . . . . . . . . . 35

2.11 View of situation where fairing does not cover nozzle geometry. . . . . 37

2.12 Results from first four geometry input parameter studies. . . . . . . . 38

2.13 Results from remaining four geometry input parameter studies. . . . . . . 40 
3.1 Ariane 4 flight profile. Derived from Turner [39] . . . . . . . . . . 42

3.2 Example axisymmetric panel geometry along symmetry plane $1 \ldots 46$

3.3 Example $2 \mathrm{D}$ fairing panel geometry . . . . . . . . . . . . 46

3.4 Body contour surface panels with control points . . . . . . . . . . 47

3.5 Surface panel geometry . . . . . . . . . . . . . . . 48

3.6 Breakdown of panel $j$ into $N_{i j_{s e g}}$ sub-segments. $N_{i j_{s e g}}=6 . \ldots \ldots$. . . . . 49

3.7 Sub-element breakdown of $i$-th panel . . . . . . . . . . . . 52

3.8 Air intake cross-section view . . . . . . . . . . . . . . . . . 54

3.9 Air intake panel geometry with constant diameter after body sections. . . 55

3.10 Panel method combination procedure flow situations. . . . . . . . 56

3.11 Zoomed in view of cowl leading edge panel geometry. . . . . . . . . 57

3.12 Streamtube bounds for different $F C \ldots \ldots$. . . . . . . . . 60

3.13 Streamtube with no-slip walls and streamtube element $\ldots \ldots$. . . . . 68

3.14 Total pressure loss along air intake surfaces. . . . . . . . . . . . . 70

3.15 Results from panel sensitivity study. . . . . . . . . . . . . . 73

3.16 Mach number distribution on cowl leading edge surfaces. . . . . . . . . . 74

3.17 Fox-Kline [46] flow regime plot with performance points for two air intakes. 75

3.18 Intake geometries from Fox-Kline plot. . . . . . . . . . . . . 75

3.19 Cowl lip with flow stagnating on outer cowl surface. . . . . . . . . . 77

3.20 Plot of $M_{\text {peak }} / M_{2}$ vs. $C R$ using potential flow methods by Seddon [18]. . 77

3.21 Intake geometries $\mathrm{C}$ and $\mathrm{D}$ with boundary layer shown for flight condition 1. 79

$3.22 \Upsilon_{A}$ for intake geometries $\mathrm{C}$ and $\mathrm{D}$ for 3 flight conditions. . . . . . . 79

4.1 RBCC rocket ejector engine diagram. . . . . . . . . . . . . . 82

4.2 RBCC rocket-ejector engine diagrams for 1-5 flight conditions. . . . . . . 83

4.3 Flow analysis results for selected geometry over 5 flight conditions. . . . 86

4.4 Air intake geometry with boundary layer shown for flight condition 4. . . 87

5.1 Computational domain used in CFD simulations along symmetry plane 1.91

5.2 Top view of computational domain used in CFD simulations. . . . . . . . 91

5.3 View of computational mesh along symmetry plane $1 \ldots \ldots 3$ 
5.4 View of computational mesh along symmetry plane $2 \ldots \ldots \ldots$. . . . 94

5.5 Mean Mach number distribution for three grids. . . . . . . . . . . . 95

5.6 RMS residuals history for the flight condition 3 simulation. . . . . . . 96

5.7 CFD simulation results with locations of high residuals highlighted. . . . 97

5.8 Monitor point history for flight condition 3 simulation. . . . . . . . . 98

5.9 Comparison of design method and CFD static pressure distributions. . . 100

5.10 Comparison of design method and CFD $\delta$ on the intake surfaces . . . 102

5.11 Boundary layer thickness along centre body at flight condition 3 . . . . 103

5.12 CFD velocity contour with CFD and design method separation locations. 104

5.13 Flow condition 1 flow feature around cowl lip. . . . . . . . . . . . 105

5.14 Percent difference of design method $A_{3}^{(\text {visc) }}$ from CFD $A_{3}^{(v i s c)} \ldots \ldots 106$

5.15 Total pressure loss distrubutions. . . . . . . . . . . . . . 107

5.16 Percent difference of design method $P_{0_{3}}^{(v i s c)}$ from CFD $P_{0_{3}}$. . . . . . 109

5.17 Comparison of CFD $\alpha$ to design method $\alpha$ with $\Delta P_{0}$ corrections. . . . 110

5.18 Percent difference of design method $\dot{m}$ from CFD $\dot{m}$. . . . . . . . . 112

5.19 Flow capture ratio vs. mass flow rate error. . . . . . . . . . . . . 112 


\section{Nomenclature}

\begin{tabular}{|c|c|c|c|}
\hline$a$ & sonic velocity $(\mathrm{m} / \mathrm{s})$ & $l_{a}$ & ellipse semi-major length (m) \\
\hline$a_{0}$ & total sonic velocity $(\mathrm{m} / \mathrm{s})$ & $l_{b}$ & ellipse semi-minor length (m) \\
\hline$A$ & flow area $\left(m^{2}\right)$ & $l_{\text {panel }}$ & length of surface panel (m) \\
\hline$A_{2-3}$ & flow area distribution & $\ell$ & control volume element length (m) \\
\hline$A R$ & $\begin{array}{l}\text { duct inflow to outflow area ratio, } \\
A_{2} / A_{3}\end{array}$ & $\dot{m}$ & air mass flow rate $(\mathrm{kg} / \mathrm{s})$ \\
\hline$c$ & chord length (m) & $M$ & Mach number \\
\hline$\tilde{c}$ & chord length ratio & $\hat{n}$ & $\begin{array}{l}\text { unit vector normal to surface panel } \\
\text { control point }\end{array}$ \\
\hline$C_{f}$ & local skin friction coefficient & $N$ & total number of surface panels \\
\hline$C R$ & cowl lip contraction ratio & $N_{C B C}$ & $\begin{array}{l}\text { number of panels used in center } \\
\text { body in region } \mathrm{C}\end{array}$ \\
\hline$d$ & centre sub-element half length $(\mathrm{m})$ & $p$ & static pressure $(\mathrm{Pa})$ \\
\hline$E$ & entrainment rate & $P_{0}$ & total pressure $(\mathrm{Pa})$ \\
\hline$F$ & force $(N)$ & $\Delta P_{0}$ & $\begin{array}{l}\text { change in total pressure at plane } 3 \\
\text { due to viscous effects }\end{array}$ \\
\hline$F C$ & intake flow capture ratio & $q$ & dynamic pressure $(\mathrm{Pa}), \frac{1}{2} \rho U^{2}$ \\
\hline$h$ & height (m) & $r$ & radius of intake surface $(\mathrm{m})$ \\
\hline$\hbar$ & modified shape factor & $r_{\min }$ & $\begin{array}{l}\text { minimum distance between control } \\
\text { point } i \text { and panel end point } j(\mathrm{~m})\end{array}$ \\
\hline$H$ & conventional shape factor, $\delta^{*} / \theta$ & $\tilde{r}$ & radius ratio \\
\hline$l_{D}$ & centre body region D length $(\mathrm{m})$ & $\hat{r}$ & unit vector in radial direction \\
\hline
\end{tabular}




\begin{tabular}{|c|c|c|c|}
\hline$s$ & $\begin{array}{l}\text { surface distance along intake sur- } \\
\text { face from stagnation point }(\mathrm{m})\end{array}$ & $\gamma$ & specific heat ratio for air \\
\hline$t$ & thickness (m) & $\Gamma$ & ring-vortex strength \\
\hline$\tilde{t}$ & thickness ratio & $\eta$ & $\begin{array}{l}\text { r-coordinate of panel subelement } \\
(\mathrm{m})\end{array}$ \\
\hline$T$ & temperature $(\mathrm{K})$ & $\varphi_{(1)}, \varphi_{(2)}$ & combination coefficients \\
\hline$T_{0}$ & total temperature $(\mathrm{K})$ & $\kappa$ & Von Karman coefficient \\
\hline$u, \vec{u}$ & $\begin{array}{l}\text { disturbance velocity and velocity } \\
\text { vector }(\mathrm{m} / \mathrm{s})\end{array}$ & $\lambda, \lambda^{\prime}$ & $\begin{array}{l}\text { ring-source strength, first derivative } \\
\text { of ring-source strength }\end{array}$ \\
\hline$U, \bar{U}, \vec{U}$ & $\begin{array}{l}\text { velocity, average velocity, velocity } \\
\text { vector }(\mathrm{m} / \mathrm{s})\end{array}$ & $\Lambda$ & boundary layer parameter, $\delta^{*} / \delta$ \\
\hline$V, \vec{V}$ & $\begin{array}{l}\text { velocity influence coefficient, ve- } \\
\text { locity influence coefficient vector }\end{array}$ & $\theta$ & momentum thickness (m) \\
\hline$x$ & $\mathrm{x}$ component $(\mathrm{m})$ & $\rho, \rho_{0}$ & density, total density $\left(\mathrm{kg} / \mathrm{m}^{3}\right)$ \\
\hline$y$ & y component $(\mathrm{m})$ & $\sigma$ & rocket to total area ratio \\
\hline$z$ & depth $(m)$ & $\zeta$ & $\begin{array}{l}\text { surface length along panel from } \\
\text { control point }(\mathrm{m})\end{array}$ \\
\hline$\tilde{z}$ & depth ratio & $\xi$ & $\begin{array}{l}\text { z-coordinate of panel subelement } \\
(\mathrm{m})\end{array}$ \\
\hline$\hat{z}$ & unit vector in $z$ direction & $\psi$ & complete elliptic integral variable \\
\hline$\Upsilon_{A}, \Upsilon_{m}$ & $\begin{array}{l}\text { percent loss in flow area, percent } \\
\text { loss in mass flow rate }\end{array}$ & & \\
\hline \multicolumn{4}{|l|}{ Symbols } \\
\hline$\alpha$ & $\begin{array}{l}\text { entrained air to rocket exhaust mass } \\
\text { flow rate ratio }\end{array}$ & $\Xi_{T}$ & skin friction parameter \\
\hline$\beta$ & surface panel tangent angle (rads) & Subscripts & \\
\hline$\chi$ & nozzle half angle (rads) & 1 & plane 1 \\
\hline$\delta, \delta^{*}$ & $\begin{array}{l}\text { boundary layer thickness, displace- } \\
\text { ment thickness (m) }\end{array}$ & 2 & plane 2 \\
\hline$\Phi$ & thrust augmentation ratio & 3 & plane 3 \\
\hline$\zeta$ & surface perimeter length (m) & Alt & flight altitude \\
\hline
\end{tabular}




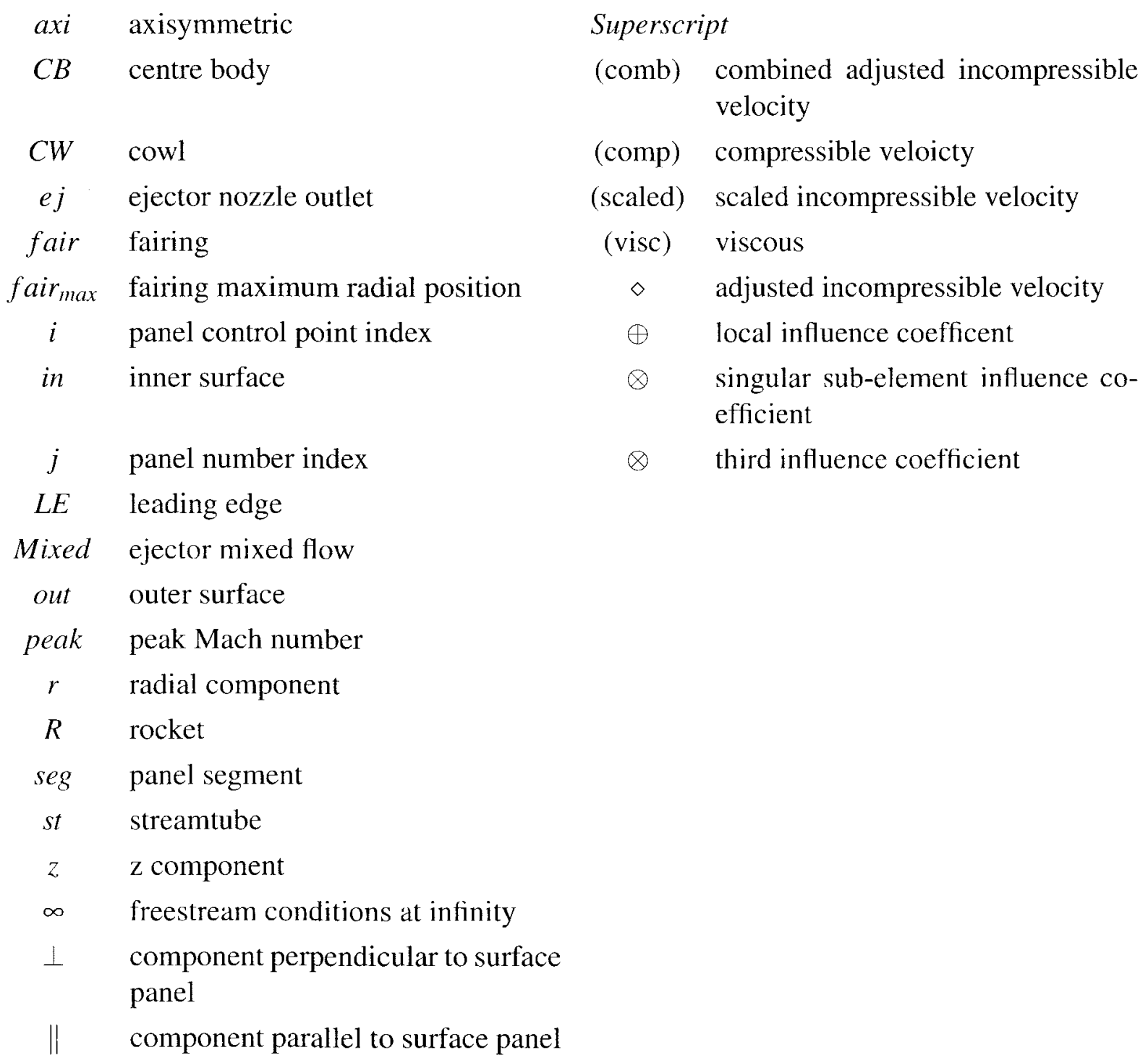

\section{Superscript}

(comb) combined adjusted incompressible velocity

(comp) compressible veloicty

(scaled) scaled incompressible velocity (visc) viscous

$\diamond \quad$ adjusted incompressible velocity

$\oplus \quad$ local influence coefficent

$\otimes \quad$ singular sub-element influence coefficient

$\otimes \quad$ third influence coefficient 


\section{Chapter 1}

\section{Introduction}

EDUCing the cost of access to space is currently one of the primary goals in launch
vehicle research. The size of the launch provider industry is relatively small with a total revenue in 2008 for the entire industry of approximately US $\$ 1.97$ billion [1], whereas general merchandisers such as Wal-Mart made about US\$405 billion in revenues [2]. The relatively small amount of revenue in the launch provider industry combined with net profits over sales typically less than $0.3 \%$ [1] makes investment in this industry unattractive. In addition, there is an over supply of launch capacity in the industry. In 2004, there was a capacity for 78 launches per year yet a demand for only 15 launches [3]. In the period between 2009 and 2018, the number of commercial space launches has been predicted by the The Federal Aviation Administration's Office of Commercial Space Transportation (FAA/AST) and the Commercial Space Transportation Advisory Committee (COMSTAC) to be about 26.7 launches per year [1].

In order to increase profit margins and remain competitive in the industry, costs must be reduced. One method of reducing costs is to reduce the number of stages used. Using a single-stage to orbit (SSTO) vehicle instead of a two-stage to orbit (TSTO) vehicle could reduce costs by up to $22 \%$ [4]. A reusable SSTO would offer additional cost savings. 
Two examples of reusable SSTO lanuch vehicle concepts are the NASA GTX vehicle concept discussed by DeBonis et. al [5] as well as the Synerjet propulsion system described by Escher [6]. Both vehicle concepts utilize rocket-based combined-cycle (RBCC) engines to enable the use of a reusable SSTO.

\subsection{Rocket-Based Combined-Cycle Engines}

Current space-launch vehicles use rocket engines for propulsion because they are effective over the entire flight, from the point of launch, all the way to orbit. However, the use of a chemical rocket engine means the launch vehicle has a more or less constant specific impulse, a measure of engine efficiency, over the entire flight. Specific impulse describes the amount of propellant required for a given change in impulse and is calculated as follows

$$
I s p=\frac{F_{\text {thrust }}}{g_{0}} \frac{\Delta t}{\Delta m}
$$

where $F_{\text {thrust }}$ is the engine thrust, $\frac{\Delta m}{\Delta t}$ is the propellant mass flow rate, and $g_{0}$ is the acceleration of gravity. Engines with higher specific impulse use less propellant mass for a given amount of thrust. Chemical rocket engines have relatively low values of Isp compared to airbreathing engines such as gas turbines or ramjets. Airbreathing engines have higher Isp's than chemical rockets since the oxidizer used for combustion is taken from the atmosphere and is not carried onboard as propellant. Figure 1.1 shows a comparison of $I s p$ values for a typical rocket engine as well as several types of airbreathing engines.

From figure 1.1, it is evident that while airbreathing engine cycles are capable of operating at much higher Isp than rocket engines, these engine cycles are limited to operate within small ranges of Mach numbers relative to the range of Mach numbers experienced during the launch vehicle's ascent. 


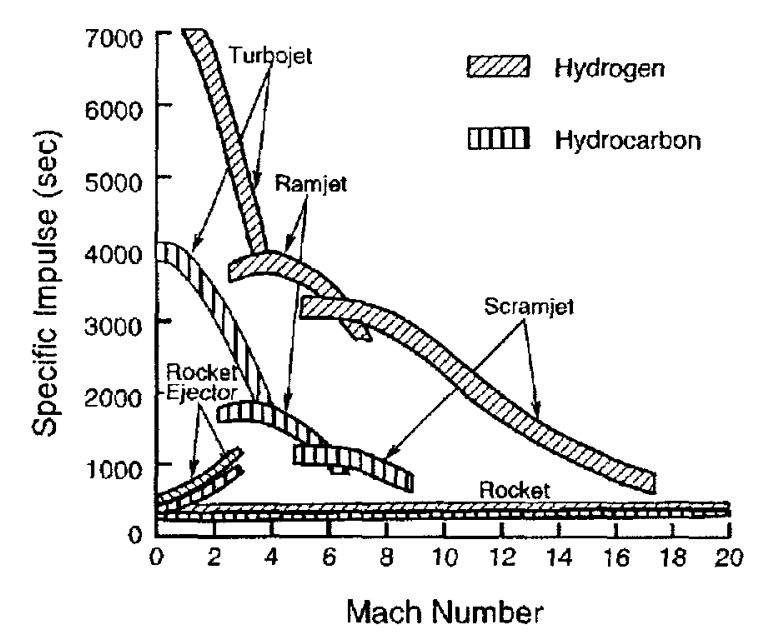

Figure 1.1: Typical specific impulse performance for various propulsion cycles. Ref [7]

Rocket-based combined-cycle engines combine a rocket engine with one or more airbreathing cycles in order to utilize the high Isp of airbreathing engine cycles, while maintaining the ability to operate over a large range of Mach numbers. A RBCC engine may initially operate in a rocket-ejector cycle from launch up to about Mach 2. The RBCC engine will then transition into ramjet mode, and then switch to scramjet mode as the flight Mach number increases. In the final portion of the flight, as the vehicle leaves the atmosphere, the RBCC engine will switch to operate in pure rocket mode. Transitioning between different engine cycles as flight speed changes allows the RBCC engine to maintain a high $I s p$ over the entire flight. An example of what a RBCC engine may look like is shown in figure 1.2 .

An ejector-scramjet RBCC engine, shown in figure 1.2, consists of rocket engines, an air intake, a mixing duct, a combustion section with fuel injectors, and finally the nozzle. When operating in rocket-ejector mode, RBCC engines use the jet pumping effect of the rocket exhaust to entrain and compress atmospheric air into the mixing duct or ejector section. The entrained air and rocket exhaust streams mix and increase the total mass flow rate through the engine, which results in thrust augmentation over a pure rocket. The magnitude of this thrust augmentation is in part dependant on the ratio between the 


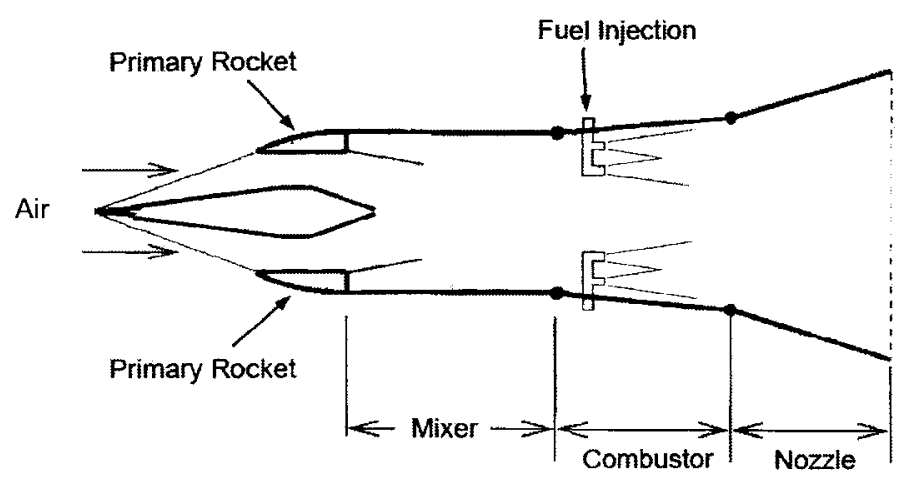

Figure 1.2: Schematic of an RBCC engine.

mass flow rates of the entrained air and the rocket exhaust, $\alpha$, where thrust augmentation increases with increasing $\alpha$ as described by Etele et. al [8]. The importance of the rocketejector cycle on the overall vehicle performance has been illustrated by Foster et. al [9] who showed that the amount of payload an RBCC powered vehicle can carry is highly dependant on the thrust augmentation produced by the rocket-ejector cycle.

An important consideration in the design of an RBCC engine operating in rocketejector mode is the length required to fully mix the rocket exhaust and entrained air streams. A rocket-ejector configuration which uses a single, centerline mounted rocket engine with a circular exhaust flow area requires a mixing duct length-to-diameter ratio of between 8 to $10[10,11]$. A mixing duct length-to-diameter ratio of 8 to 10 is impractical as the mixing duct mass would become prohibitive and limit the vehicle payload. Therefore additional performance gains can be obtained by reducing the required mixing length of the rocket exhaust and air streams. A reduced mixing length allows for a reduced mixing duct length and therefore reduces the vehicle mass. This reduction in vehicle mass could increase profit margins by allowing the vehicle to carry more payload or less fuel.

The mixing of two fluid streams occurs primarily due to the viscous shear forces which act along the shear area between the streams. A reduction in mixing length can be achieved through an increase in the shear layer area between the rocket exhaust stream and the entrained air stream. One method of increasing the shear layer area between the 

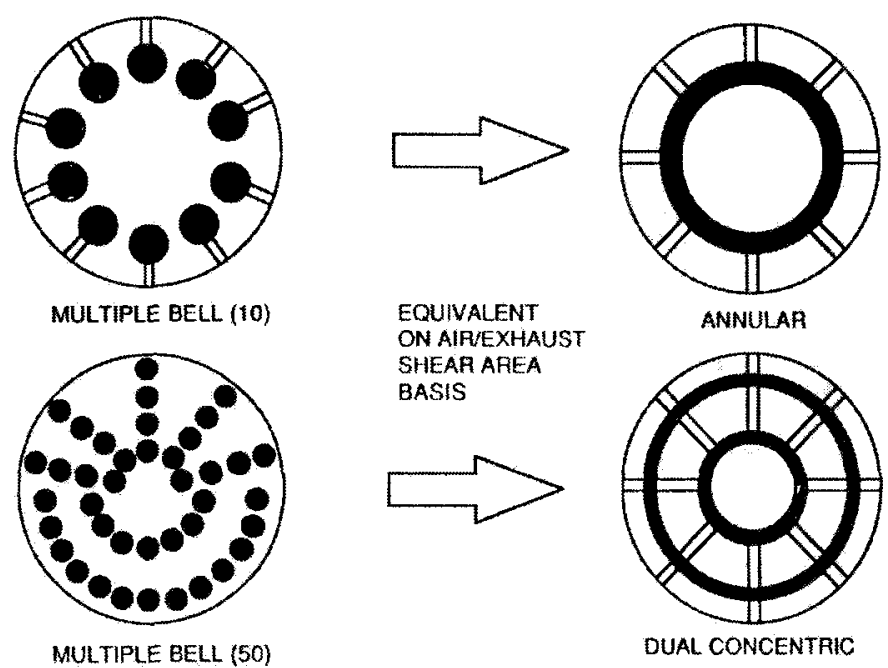

Figure 1.3: Proposed rocket exhaust configurations by Escher and Schnurstein. Ref [12]

two streams is to significantly alter the way the rocket exhaust is distributed. Escher and Schnurstein [12] suggest several configurations, shown in figure 1.3, such as distributing the rocket exhaust among several small rocket engines, or arranging the rocket exhaust with annular or dual concentric exhaust areas. The annular exhaust area and the dual concentric configurations are expected to require mixing duct length-to-diameter ratios of about 2.5 and 1 respectively. While these rocket configurations greatly reduce mixing duct length-to-diameter ratios, they would require the use of several small rocket engines and coordination between them. Development of these rocket engines would add to the launch vehicle costs. In order to reduce development costs it would be desirable to reduce mixing lengths while maintaining a circular throat area so that a single large rocket engine can be used.

To date, RBCC research has focused on feasibility studies, computational flow analysis, and ground testing. Engine testing performed in the 1960 s on rectilinear and axisymmetric ejector-scramjet RBCC engines has demonstrated that smooth transitions between engine cycles are possible [7]. Flight path studies have been conducted for SSTO RBCC vehicles by Nakane et. al [13]. Several different RBCC engine geometries have 

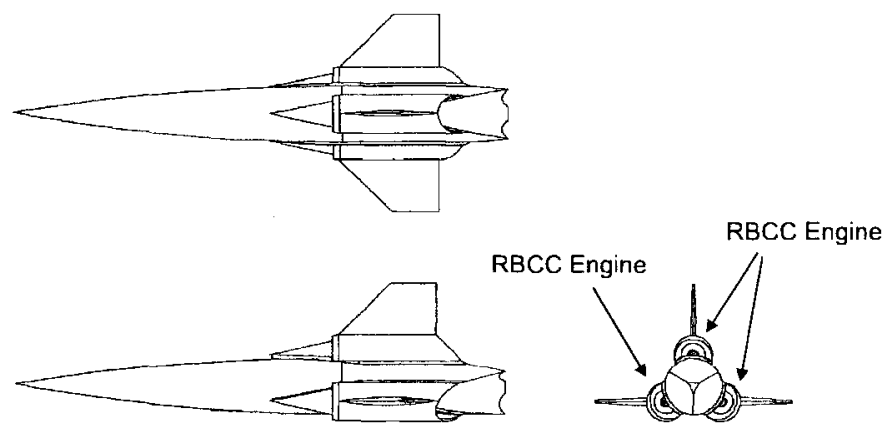

Figure 1.4: Three-view of NASA GTX concept vehicle. Ref [5]

been proposed or tested, and include axisymmetric geometries with a centre line mounted rocket [7], axisymmetric geometries with a several small distributed rockets as proposed by Escher [6], a semi-axisymmetric geometry used on the NASA GTX concept vehicle $[5,14]$, shown in figure 1.4 , and a strutjet engine which has a rectilinear geometry and uses rockets mounted within struts [15].

\subsection{Novel RBCC Rocket Nozzle}

A novel type of RBCC rocket nozzle geometry, shown in figure 1.5, has been proposed by Cerantola and Etele $[16,17]$ with the goal of reducing ejector mixing lengths by increasing the shear layer area of the rocket exhaust stream while retaining a circular throat area. Maintaining a circular throat area allows this rocket nozzle geometry to be adapted to existing rocket engines. This novel rocket nozzle also incorporates openings for air intakes into its geometry which allow air to be entrained into the centre of the mixing duct. The openings in the nozzle create a nozzle geometry which is separated into segments or clovers, shown in figure 1.5, which are ducts for the rocket exhaust. A section view of the rocket nozzle geometry shown in figure 1.6 shows that the rocket exhaust enters through a common throat, but is then directed through the nozzle segments and then exits at the outflow plane of each segment. The shape of the nozzle segments can be adjusted 

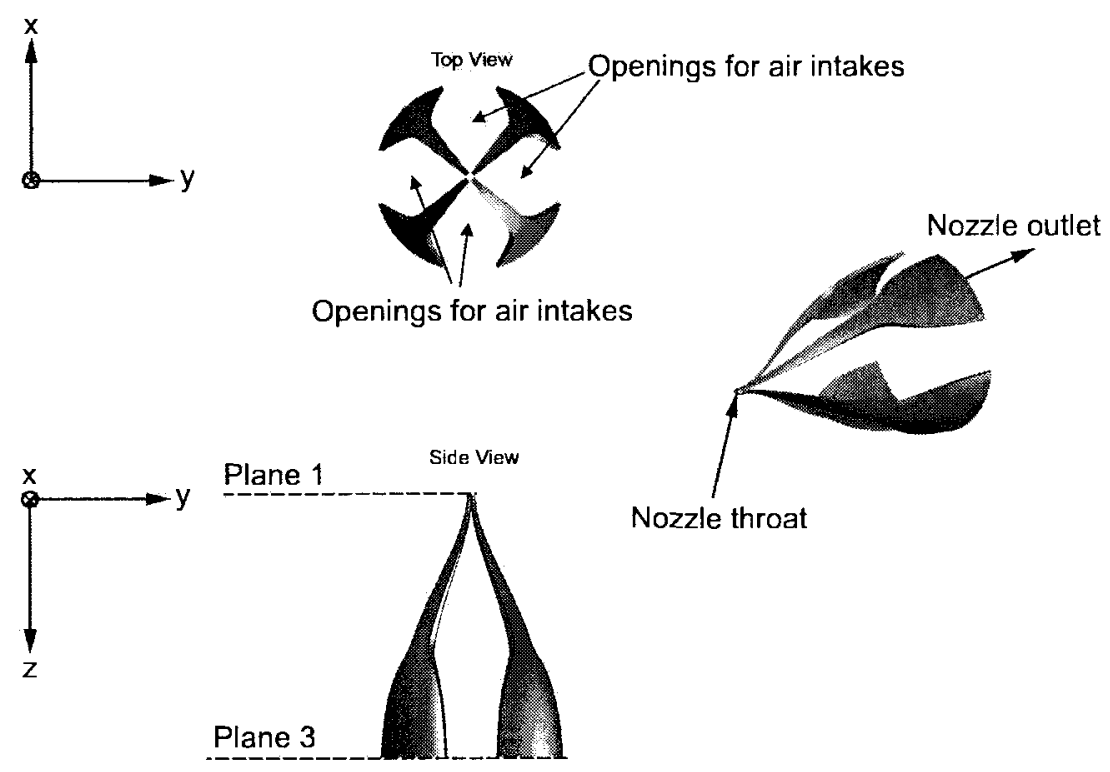

Figure 1.5: Example of novel RBCC rocket nozzle geometry.

to approach an annular exhaust area. The annular rocket exhaust area combined with the openings that allow air to be entrained into the mixing duct act to increase the shear layer area between the two flow streams and therefore reduces the required mixing length. This nozzle geometry would be positioned upstream of the mixer duct in an RBCC engine as shown in figure 1.7. Note that the nozzle geometry shown is not the entire rocket engine, a rocket engine combustion chamber is also required and would be located upstream of the nozzle geometry as shown in figure 1.7 .

The design method for this novel rocket nozzle geometry, developed by Cerantola [17] allows for variation of the shape, size, and number of air intake openings in the nozzle. Several possible nozzle geometries are shown in figure 1.8.

There are several design challenges associated with this novel nozzle geometry. Air entrained through the current unmodified nozzle geometry would likely separate and cause significant losses in total pressure as well as air mass flow rate. A reduction in air mass flow rate would lower $\alpha$, the entrained air to rocket exhaust mass flow rate, and thus thrust augmentation. For this rocket nozzle geometry to perform efficiently in an RBCC 


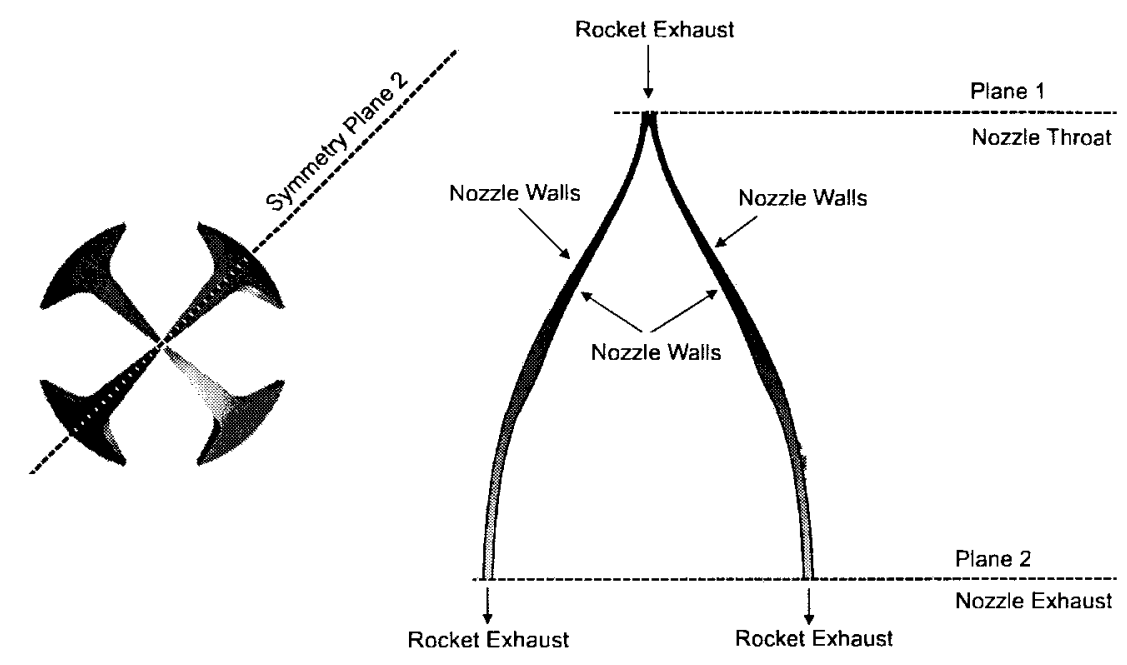

Figure 1.6: Section view of nozzle along symmetry plane 2.

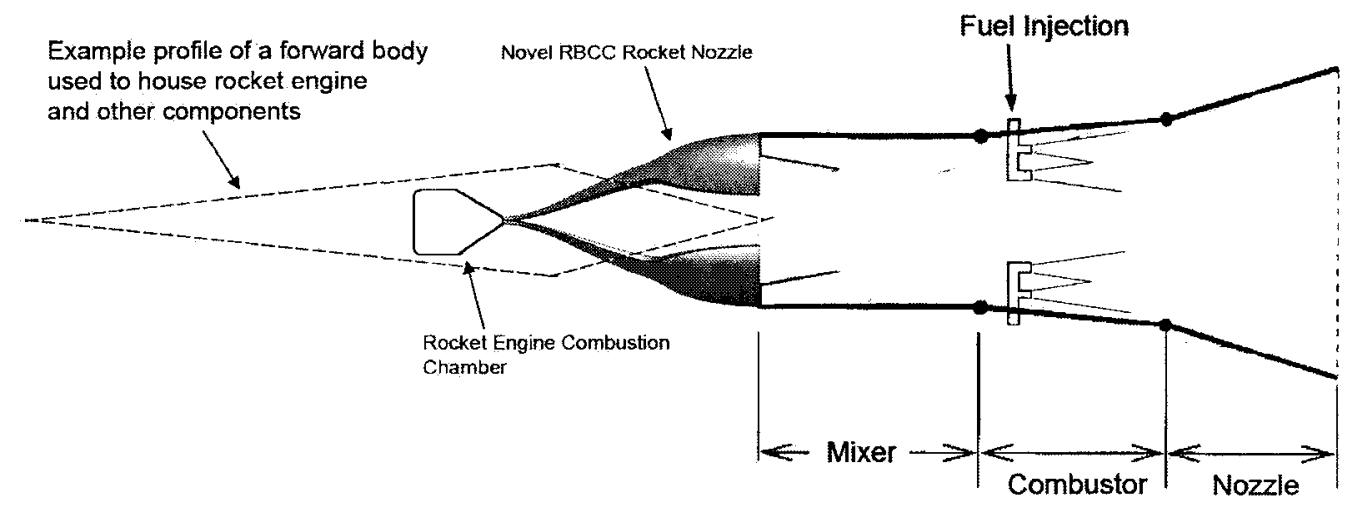

Figure 1.7: View of RBCC engine with novel RBCC rocket nozzle.
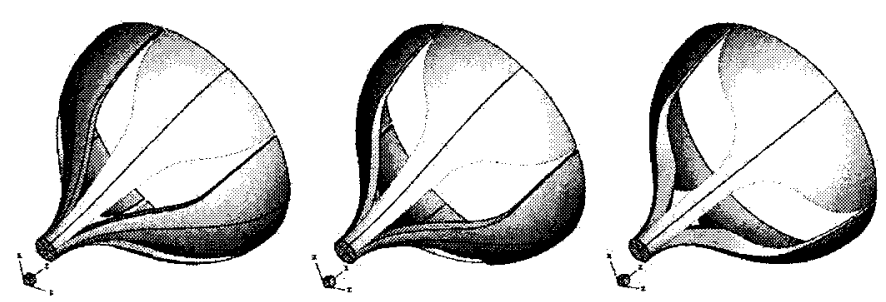

Figure 1.8: Example of rocket nozzle geometry variation. 
vehicle the current nozzle geometry must be modified to ensure the air mass flow rate is maximized and total pressure losses are minimized. Another design challenge with this nozzle is that portions of the nozzle geometry are very thin and would likely need some structural support.

\subsection{Objectives}

The novel RBCC rocket nozzle design method developed by Cerantola represents the first stage in the development of a practical $\mathrm{RBCC}$ rocket nozzle that minimizes mixing duct length while maintaining a circular throat area. The current rocket nozzle geometry contains openings which allow air to be entrained through the center of the mixing duct. The purpose of an air intake as stated by Seddon and Goldsmith [18] is to ensure that "...an aircraft engine is properly supplied with air under all conditions of aircraft operation and that the aptitude of the airframe is not unduly impaired in the process." This thesis interprets the term "properly supplied" to mean the air intake is designed to supply the engine with air at the desired pressure while minimizing total pressure losses and flow area blockages. Air entrained through the current openings in the rocket nozzle would likely experience significant regions of flow separation and cause high total pressure and air mass flow rate losses. The current openings in the rocket nozzle will therefore not properly supply the air intake with air. It is clear that a proper air intake geometry needs to be designed for this rocket nozzle geometry.

Keeping the above air intake requirements in mind, the present research represents the next phase in the development of this novel RBCC rocket nozzle. The goal of the present research is to develop an air intake design method for this novel rocket nozzle geometry. The two primary objectives of this design method are to generate a 3D air intake geometry for a given rocket nozzle geometry while giving the designer significant control over the air intake geometry shape, as well as to provide an initial performance prediction of the air 
intake geometry operating at given flight conditions. This initial performance prediction can then be used to determine if modifications to the air intake geometry are necessary.

The first objective is achieved by using a set of geometry input parameters along with a given rocket nozzle geometry to generate a $3 \mathrm{D}$ air intake geometry. The given rocket nozzle geometry is sized to meet specified performance parameters and the geometry input parameters are selected to allow the user to adjust the intake geometry to meet performance and geometric requirements.

The second objective is achieved by using a simple flow analysis method on the air intake geometry to predict air mass flow rate through the air intake. Air mass flow rate is chosen as a metric to evaluate air intake performance since it has been shown that the ratio between air and rocket exhaust mass flow rate significantly affects rocket-ejector cycle performance. Based on the flow analysis results, a designer can then modify the geometry input parameters until the flow analysis predicts acceptable performance. Alternatively, the performance of an air intake geometry over a range of flight conditions can be estimated by running the flow analysis using different flow condition parameters.

The flow analysis used to predict air mass flow rate combines an inviscid flow analysis with a viscous flow analysis to account for boundary layer growth, boundary layer separation, and total pressure losses. Although $\mathrm{RBCC}$ vehicles are required to operate over a large range of Mach numbers, the flow analysis is currently limited to subsonic flight speeds. By focusing on improving the performance of the rocket-ejector cycle, the overall performance of the RBCC engine vehicle will be improved since it has been shown that improving the performance of this engine cycle has a significant effect on the amount of payload an RBCC vehicle can carry.

An additional constraint placed on the air intake design method is that it is required to run relatively quickly since it is intended for use as an initial design tool and it will be used as a component of a geometry optimization tool in the future. This optimization tool will 
need to run the air intake design method several times and a long run time for the design method would limit the usefulness of the optimization tool.

\subsection{Intake Analysis Literature Review}

The following sections describe various methods of predicting the flow through air intakes. Because the flow analysis is currently restricted to subsonic flight speeds, the analysis methods described here are intended for subsonic flows.

In the 1960s, Sovran and Klomp [19] summarized the data from many subsonic diffuser experiments and developed correlations between diffuser geometry and total pressure loss. While these correlations provide reasonable performance predictions, they are only applicable to relatively simple geometries (straight walled planar, conical, or annular diffusers) operating at low subsonic speeds. To predict the behaviour of more complex diffuser or intake geometries, scale model tests in wind tunnels had been used as the primary design tool. Wind tunnel experiments can be time-consuming and expensive. Due to these limitations, several theoretical flow analysis methods with applications to air intakes have been developed. Some of these methods require relatively little time and resources and are therefore well suited to use during the initial phases of an intake design. There are three primary methods used for intake flow prediction: non-interacting inviscid-viscous methods, interacting inviscid-viscous methods, and full Navier-Stokes solvers. The first two analysis methods are zonal methods and separate the flow into an inviscid core region and a viscous flow region. The difference between these two methods is in the way the inviscid region and the viscous region interact. The following sections will describe the various analysis methods for solving the inviscid and viscous flow regions in an air intake, the interaction methods for these flow regions, and the full Navier-Stokes solvers. 


\subsubsection{Inviscid Flow Solution Methods}

There are three common methods used for solving 2D inviscid flow fields through intakes: 1D inviscid core methods, potential flow panel methods, and field methods. The 1D inviscid core method assumes that the intake is symmetrical about a straight centre line and does not contain a centre body. As long as the cross-sectional area of the intake is known at all axial positions, the static pressure distribution along the intake wall can be calculated using the continuity equation. Most applications of the $1 \mathrm{D}$ inviscid core method are applied to simple intake geometries that are symmetrical about a straight centre line. However, the 1D inviscid core method has been modified by Childs [20] for intakes with curved centre lines such as 'S' shaped ducts.

\section{Inviscid Panel Methods}

Perhaps the most popular method of solving the inviscid flow field around 2D external bodies is through the use of a $2 \mathrm{D}$ panel method. In panel methods, the surface of a body is approximated with panels that contain potential flow elements. The strength of each potential flow element is set so that the local velocity normal to each panel is zero. Panel methods have the advantage of being relatively fast running, easy to implement and accurate for incompressible flows. The first practical panel method was presented by Hess and Smith [21]. Panel methods can vary in what type of potential flow elements they use, as well as how the surface panels are shaped. Depending on what type of flow one wishes to model, panel methods can use sources or vortices [22] placed at the centre of each surface panel, a combination of sources and vortices [21,23-25], or a combination of doublet's and sources [26]. The strength distribution of each potential element along the panels can also vary. Some methods use constant strength elements [21,22], while others use linear or quadratic distributions for improved accuracy [23-25]. Accuracy can also be improved by modifying the shape of each surface panel. Simple panel methods use flat 
panels [21, 22], while others use parabolic shaped panels [23-25]. Hess [24,25] showed that an axisymmetric panel method using parabolic panels, a linear source strength distribution and constant strength vortices produced excellent results for internal flow fields such as intakes. The net flux error using this method was up to 10 times lower than the errors found using a panel method with flat panels and constant strength sources. For this reason, the axisymmetric panel method which uses parabolic panels and a linear source strength distribution is used in this work.

A limitation of panel methods is that they only apply to inviscid, incompressible flows. In order to solve a compressible flow field, compressibility corrections can be applied to panel method results. Examples of compressibility corrections are the Prandtl-Glauert method, the Goethert method, and the Lieblein-Stockman method [27]. In order to apply the Prandtl-Glauert and Goethert methods, the actual body geometry must be stretched $[28,29]$. This geometry modification step would add to the computational time required for analysis and is therefore not desirable for use in the current work. The Lieblein-Stockman method was developed for internal flows and does not require any geometry stretching. All of these methods are restricted to subsonic compressible flow and do not model flow shocks.

\section{Field Methods}

An alternative to panel methods with compressibility corrections are field methods that solve the full potential equations or the Euler equations. The partial differential full potential equations or Euler equations do not assume constant density and therefore provide valid flow solutions past Mach 0.3. A pure field method that solves the Euler equations has been used by Peace [30] to accurately solve the flow through an inlet. Reddy et al [31] developed a subsonic inlet optimization procedure based on an Euler field method.

In order to solve the partial differential equations (PDEs), they are approximated with 
finite-difference or finite-volume expressions. These expressions must be solved over a grid that spans the entire flow domain. The generation of a body fitted computational grid becomes difficult relative to panel methods for complex multi-body geometries such as an air intake with a center body. Also, the solution procedure for these PDEs requires iteration and is time consuming relative to panel methods. While field methods can provide very accurate results, due to the time and resources required to solve them, they are not used in this thesis.

\subsubsection{Viscous Flow Solution Methods}

There are two types of boundary layer calculation methods: partial differential methods and integral methods. In partial differential boundary layer methods, such as those used by Cebeci et al. [32] and Liou et al. [33], the boundary layer equations are approximated by finite difference expressions. The solution of these finite difference expressions must be solved over a spacial grid placed throughout the boundary layer. Given the static pressure distribution as a boundary condition, the boundary layer velocity profile is then solved using an iterative procedure. The PDE solution method and choice of dependant and independent variables can affect the accuracy of the boundary layer solution. A disadvantage of PDE methods is that they require more computational time than integral methods and may encounter convergence problems.

Integral boundary layer methods use the momentum equation along with an auxiliary equation such as the entrainment equation to calculate the boundary layer development. Using the static pressure distribution as a boundary condition, integral values such as displacement thickness, $\delta^{*}$, and momentum thickness, $\theta$, can be calculated. These integral values are dependant on surface distance from the stagnation point, but are independent of vertical position in the boundary layer. Integral boundary layer methods have proved popular due to their relative simplicity. The boundary layer calculation is typically marched 
downstream of the stagnation point using a Runge-Kutta scheme. Head's entrainment method [34] is a commonly used turbulent boundary layer method for incompressible flows. Green [35] and Childs [20] have expanded on Heads method to include compressibility effects. Childs method is used in this work and differs from Green's method in that it uses a shape factor correlation which is explicitly dependant on Mach number.

\subsubsection{Inviscid-Viscous Interaction Methods}

Air flow through intakes can be divided into zones; an inviscid core zone, and viscous zones which contain the boundary layers. In cases where boundary layer separation occurs, the viscous zone is subdivided into attached and detached zones. These zones are illustrated in figure 1.9. The interaction between these zones can be modeled in three ways; non-interacting, weakly-interacting, and strongly-interacting. Accuracy improves as one moves from a non-interacting method to a strongly-interacting method.

The non-interacting and weakly-interacting methods are well suited to modeling high Reynolds number flows without boundary layer separation where boundary layers and thus viscous zones are very thin relative to the inviscid zone. In these flow situations, the inviscid zone is dominant and is primarily dependant on the intake geometry. The properties of the boundary layer in the viscous zone are determined based on the inviscid zone solution.

In the non-interacting method, the viscous zone is assumed to have no affect on the inviscid zone. Therefore, the inviscid flow is solved first in order to find the static pressure distribution along the intake surfaces. This pressure distribution is then used as a boundary condition for the boundary layer equations.

In the weakly-interacting method, the viscous zone affects the inviscid zone, but the inviscid zone is dominant. The standard method for calculating a weakly-interacting flow is an iterative procedure and is described below. 


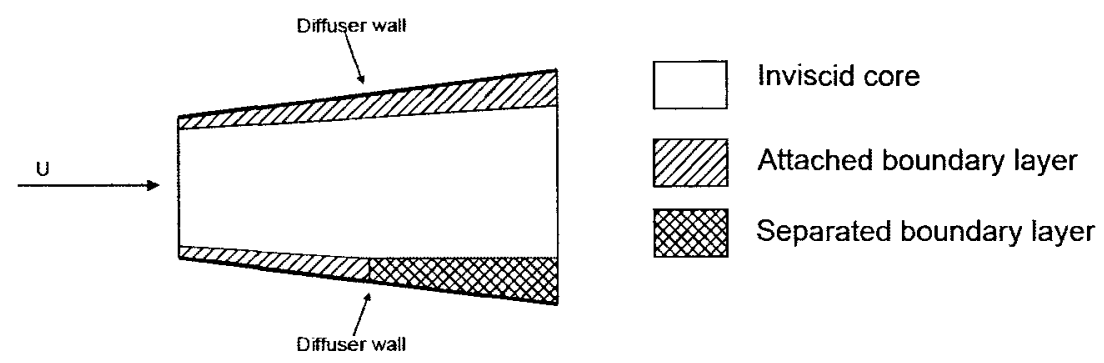

Figure 1.9: Inviscid and viscous flow zones in a diffuser

Step 1: Solve the inviscid core zone using the given flow conditions and the intake geometry.

Step 2: Use the static pressure distribution (calculated in Step 1) at the boundary between the inviscid and viscous zones as an input for the boundary layer calculations.

Step 3: Update the intake geometry using the displacement thickness results from the boundary layer calculations from Step 2. The addition of the displacement thickness changes the flow area distribution through the intake and thus slightly affects the inviscid flow solution.

Step 4: Repeat steps 1 - 3 until the displacement thickness distribution does not change.

The weakly interacting method described above is simple and has been shown to be effective for flows with thin, fully attached boundary layers.

Both the non-interacting and weakly-interacting methods are unable to model flow beyond boundary layer separation. It has been shown that boundary layer equations which use pressure as an input become singular at the point of separation [36]. Once the boundary layer equations become singular, boundary layer growth becomes un-bounded and produces non-physical results.

The use of the weakly interacting scheme downstream of the boundary layer separation point becomes invalid because as the separated boundary layer region grows, it begins to strongly influence the inviscid region and the weakly interacting method will fail to 
converge. The weakly interacting scheme is therefore of limited use for intake flows since these flows can contain regions of separated flow depending on flow conditions.

Strongly-interacting inviscid-viscous methods have been developed in order to model flows with separated boundary layers. Strongly-interacting methods solve the inviscid core equations and the boundary layer equations simultaneously. By directly coupling the equations for the inviscid and viscous flow regions, the boundary layer singularity problem can be avoided. Childs [20] and Johnston [37] have developed strong interaction methods designed specifically for internal flows such as intake flows. However, since the inviscid and viscous flow equations must be solved simultaneously and these methods employ a ID inviscid core assumption, the types of applicable diffuser shapes is limited. The air intake geometry being developed for the present work is composed of an axisymmetric centre body and a cowling. Both of these surfaces can have highly curved surfaces. The presence of the centre body and the curvature of the intake surfaces makes a 1D core assumption inaccurate. Childs suggests using a 2D core method for inviscid core flows with significant curvature. This 2D core method accounts for the cross-stream pressure gradient due to a curved inviscid core geometry. However, this 2D core assumption can not account for the flow distortion caused by intake lips or the shape of the centre body upstream of the inflow plane of the intake. The flow distortion at the inflow plane of the intake due to these geometry features can often determine if boundary layer separation will occur in an intake and thus the $2 \mathrm{D}$ core method is also not well suited to the present air intake geometry. Therefore, the strongly-interacting method is not used in this work.

The non-interacting method is used in this work due to the geometric limitations of the strongly-interacting method and the convergence problems with the weakly-interacting method when modeling flows with separated boundary layers. If separation is predicted using the non-interacting method, the boundary layer is assumed to extend straight downstream from the separation point. This assumption will tend to produce a conservative air mass flowrate prediction since modeling inviscid-viscous interaction tends to delay 
boundary layer separation.

\subsubsection{Full Navier-Stokes Solvers}

Full Navier-Stokes solvers offer the most accurate predictions of intake flows. These solutions are typically obtained from computational fluid dynamics (CFD). Commercial software packages such as CFX and FLUENT are examples of full Navier-Stokes solvers. The full Navier-Stokes equations take both compressibility and viscosity into account. In order to obtain accurate results, meshes with large numbers of computational nodes are required. The large number of computational nodes and equations that must be solved means that computational time can be on the order of days to weeks. The large computational times (or cost) required for these simulations means that they are currently not suited to use in rapid initial design tools. These solvers are better suited to the optimization of intake geometries that already provide reasonable levels of efficiency. 


\section{Chapter 2}

\section{Air Intake Design Method: Geometry Creation}

The first step in the air intake design method is to generate the air intake geometry given a fixed rocket nozzle geometry and 8 input parameters. The fixed rocket nozzle geometry provides the air intake design method with the physical coordinates of the nozzle walls. From the nozzle geometry, parameters required to define the air intake geometry such as the nozzle wall radial contours, $z_{3}$, and $r_{N Z_{3} \text { in }}$ are found (Fig 2.5). The 8 primary geometric input parameters are listed in table 2.1. The air intake geometry, shown in figure 2.1 is built around the novel rocket nozzle geometry and consists of three types of bodies, an axisymmetric centre body, a cowl and nozzle fairings. Figure 2.1 also shows where the air intake would be located relative to the rest of the $\mathrm{RBCC}$ engine components. Recalling figure 1.5 , and comparing to figure 2.1 , one can see that the nozzle geometry is embedded within portions of the centre body, fairing, and cowl intake bodies. The fairing provides structural support for the thinner portions of the rocket nozzle geometry. The purpose of the cowl and centre body geometries is to create an enclosed flow area with a controllable area distribution and surface curvature such that the RBCC engine is properly supplied with air. The purpose of the nozzle fairing is to prevent flow distortion and separation on the nozzle geometry that spans the space between the centre body and cowl, as well as to provide structural support. 

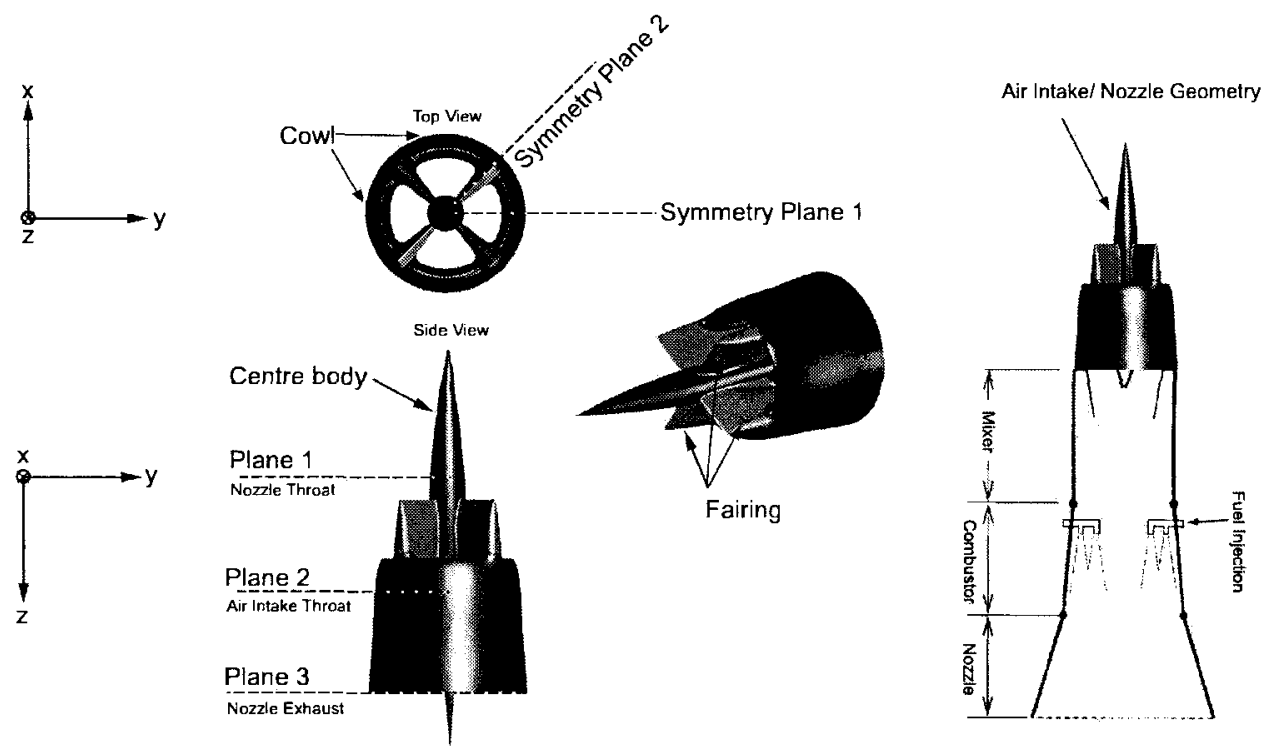

Figure 2.1: Example of novel RBCC air intake geometry

Table 2.1: Input Parameter Description

$\begin{array}{rccr}\tilde{z}_{2} & \frac{\left(z_{3}-z_{2}\right)}{z_{3}} & 2.4,2.5 & \text { duct length ratio } \\ \tilde{c}_{\text {fair }} & \frac{c_{\text {fairmax }}}{z_{3}} & 2.4,2.5 & \text { fairing profile chord ratio at max radial position } \\ \sigma & \frac{A_{R}}{A_{R}+A_{3}} & 2.5 & \text { rocket/total flow area ratio } \\ \tilde{t}_{3} & \frac{t_{3}}{r_{N Z_{3}}} & 2.5 & \text { cowl thickness ratio at plane 3 } \\ A R & \frac{A_{2}}{A_{3}} & 2.5 & \text { plane 2 - plane 3 area ratio } \\ \tilde{r}_{C B_{1}} & \frac{r_{C B_{1}}}{r_{N Z_{1}}} & 2.5 & \text { centre body radius ratio at plane 1 } \\ C R & \frac{A_{C W_{I E}}}{A_{2}} & 2.8 & \text { cowl lip contraction ratio } \\ A_{2-3} & - & 2.5 & \text { duct area distribution }\end{array}$


The 8 intake geometry parameters were chosen to allow significant control over the air intake shape. In particular, $\tilde{z}_{2}$ and $A R$ allow control over the duct length and the inlet to outlet area ratio since these characteristics are the controlling factors in diffuser performance (Sovran and Klomp [19]). The rocket/total flow area ratio, $\sigma$, is an important parameter in rocket-ejector analysis and is thus specified as a user input. The centre body radius ratio at plane $1, \tilde{r}_{C B_{1}}$, is selected as an input since it affects the shape of the centre body upstream of the duct and can have a significant effect on the intake performance at high subsonic free stream velocities. The cowl lip contraction ratio, $C R$, is an input since it has been shown to be a controlling factor in air intake performance when operating at large flow capture ratios [18]. The remaining primary input parameters give the user control over the general curvature of the center body and cowl surfaces. A set of secondary input parameters, which allow the designer to fine tune an air intake geometry, can also be changed, however, these parameters are set to default values and do not significantly alter the overall geometry. Examples of secondary input parameters, which will be discussed in the following sections, include $\ell_{\text {fair }}$, the ratio between $r_{C B_{3}}$ and $l_{D}$, the ratio between $r_{C B_{1}}$ and the centre body nose location, the ratio $l_{a} / l_{b}$ for the cowl lip, and various fillet radii used in the $3 \mathrm{D}$ geometry creation.

The origin of the coordinate system in which the intake bodies are defined is located along the air intake centre line at the rocket nozzle throat location, which is located within the centre body at plane 1 (see fig 2.1). The z-axis is parallel to the air intake centre line and is positive in the direction downstream of the nozzle throat location. Three $x-y$ planes, shown in figure 2.1, are defined to aid in the geometry definitions, with plane 1 located at the origin, and plane 2 and plane 3 located downstream of plane 1 . The location of plane 2 corresponds to the air intake throat location and can be shifted upstream or downstream using the primary input parameters such as $\tilde{z}_{2}$. The location of plane 3 corresponds to the exhaust plane of the rocket nozzle. Since the air intake is symmetric about two planes extending radially from the centre line, (also shown in figure 2.1 as symmetry planes), the 
intake bodies are defined between these two planes.

The following sections will describe the geometry creation procedure used in this design method.

\subsection{Fairing Geometry}

The nozzle fairing geometry is the first air intake body generated. As previously stated, the fairing geometry is necessary to prevent flow distortion and separation off of the nozzle geometry that spans the space between the centre body and cowl. A NACA symmetrical airfoil profile is used to define the base fairing geometry at any given radial position because its shape and position in space can be completely defined by a chord length, a thickness ratio (maximum thickness/chord), and a leading edge location. However, any symmetrical profile which can be defined using these parameters can be used in place of the NACA profile. The base fairing geometry used in this work is defined using a modified 4-digit NACA symmetrical airfoil profile (NACA 0020-34) with a fixed leading edge location over all radial positions. The second to last digit of the NACA 0020-34 profile indicates that this profile has a sharper leading edge than the standard NACA 0020 profile, and the last digit indicates that profile maximum thickness is located at the $40 \%$ chord location. As will be discussed later, the thickness ratio of the base profile is scaled at each radial position to conform to the rocket nozzle geometry. The choice of this final fairing configuration is the result of tests on several different fairing configurations, shown in figure 2.2. The views in figure 2.2 show a portion of the nozzle geometry sides along with the fairing profiles at two radial positions when looking parallel and normal to symmetry plane 2 (see figure 2.1). The centre body and cowl surfaces are not shown in figure 2.1. The fairing profile at each radial position is applied to a constant radius arc, which is why the side views in figure 2.2 appear to be curved. Fairing geometry configuration 1 consisted of a symmetrical tail profile attached to the rocket nozzle geometry, shown in 


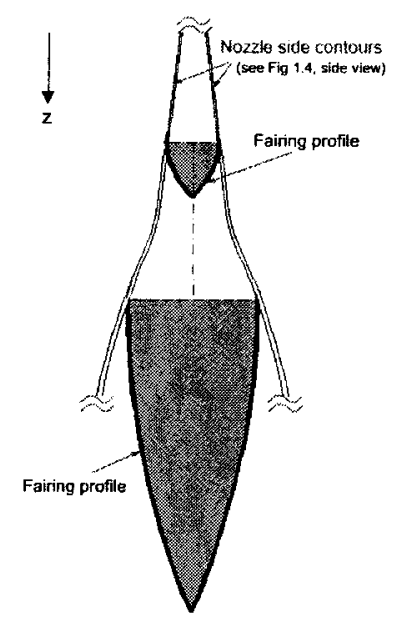

(a) Profile view of fairing configuration 1 shown parallel to symmetry plane 2 .

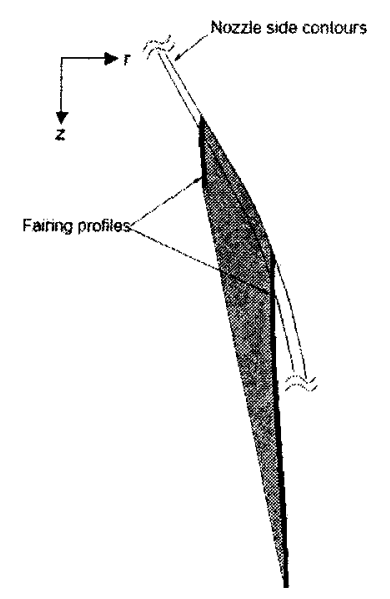

(d) Side view of fairing configuration 1 shown normal to symmetry plane 2 .

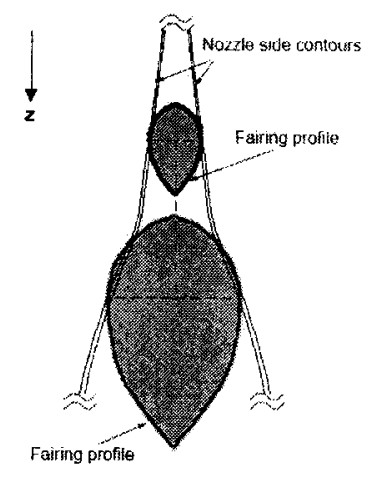

(b) Profile view of fairing configuration 2 shown parallel to symmetry plane 2 .

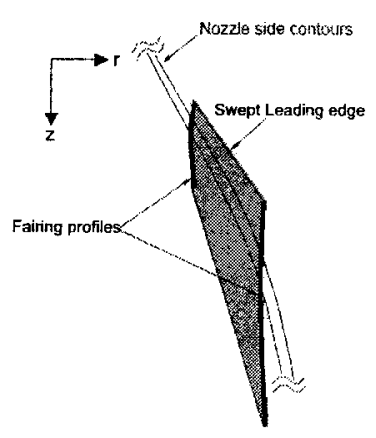

(e) Side view of fairing configuration 2 shown normal to symmetry plane 2 .

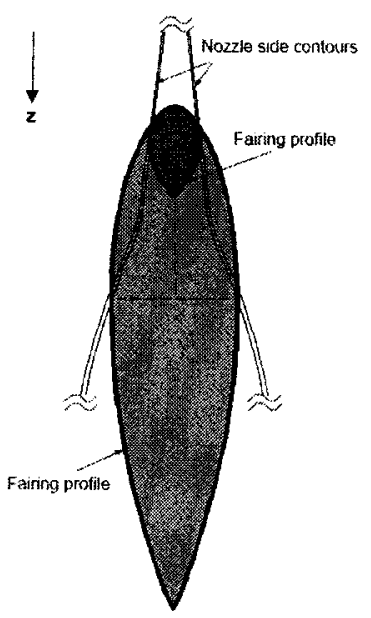

(c) Profile view of fairing configuration 3 shown parallel to symmetry plane 2 .

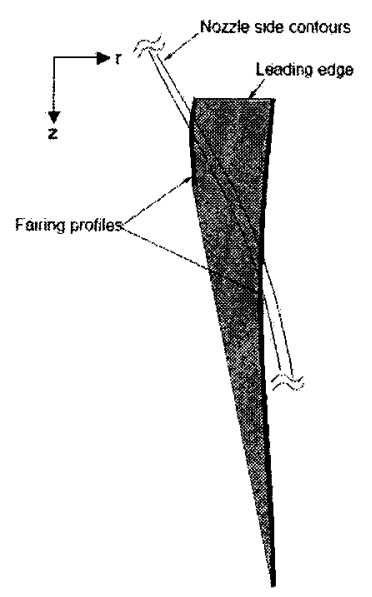

(f) Side view of fairing configuration 3 shown normal to symmetry plane 2 .

Figure 2.2: Fairing geometry profiles 
figure 2.2(a),(d). CFD results showed this profile shape was ineffective since the incoming flow tended to separate as it turned around the sharp corner of the exposed nozzle surface and was rapidly accelerated around the corner and then decelerated as it entered the duct region.

Fairing configuration 2, illustrated in figure 2.2(b),(e), used a full NACA symmetrical airfoil profile with a fixed thickness ratio so that the fairing geometry extends upstream of the rocket nozzle geometry. Including the portion of the fairing geometry upstream of the rocket nozzle geometry greatly reduced the amount of flow separation along the fairing. However, due to the varying thickness and location of the nozzle geometry and the fixed thickness ratio of the fairing profile, the resulting fairing geometry had a swept leading edge. This swept fairing geometry induced an outward radial velocity in the flow in CFD simulations and the flow along the fairing tended to be pushed radially towards the cowl surface. Along the intersection region between the cowl and the fairing surfaces, the fairing surface streamlines and the cowl surface streamlines interacted to form a region of swirling flow. This region of swirling flow can grow and was found to have a negative impact on air mass flow rate through the intake.

Fairing configuration 3, figure 2.2(c),(f), is the final configuration and addresses the problems with the previous two configurations by using a symmetrical 4-digit NACA series airfoil with a constant leading edge location at all radial positions. Maintaining a constant leading edge location along the fairing reduces the radial velocity of the flow along the fairing and reduces the size of the region of swirling flow.

The fairing geometry is created in two phases because it is desirable to have explicit control over the flow areas at plane 2 and plane 3 , and all three intake bodies influence the flow area at these planes. The first phase develops the relationship between fairing chord, $c_{\text {fair }}$, and thickness ratio, $\tilde{t}_{\text {fair }}$, at any radial position. This information is then used to generate the centre body and cowl geometries. The second phase generates the final 3D fairing geometry accounting for the centre body and cowl. 


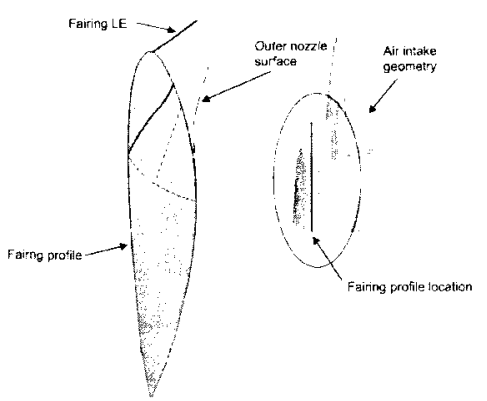

(a) Fairing profile at maximum radial position.

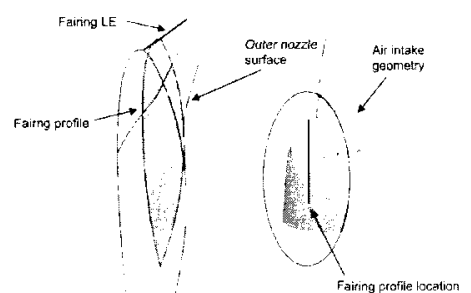

(b) Fairing profile at middle radial position.

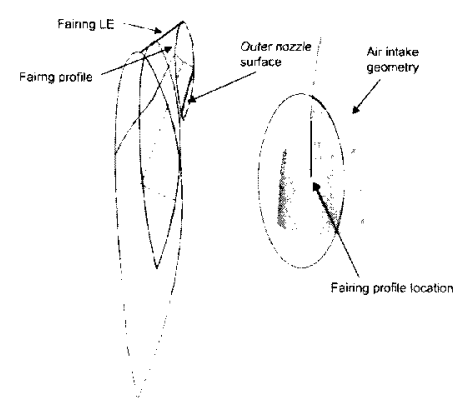

(c) Fairing profile at inner radial position.

Figure 2.3: Fairing construction sequence.

In the first phase, the two geometry input parameters, $\tilde{z}_{2}$ and $\tilde{c}_{\text {fair }}$ max , along with the existing rocket nozzle geometry are required to define the profile of the fairing geometry. The fairing profile at the outer most radial position of the fairing is defined using these inputs. The fairing profile at any remaining radial position is then defined based on the rocket nozzle geometry and the leading edge location of the outer most fairing profile. The sequence used to build the fairing geometry is illustrated in figure 2.3(a)-(c).

The outer most radial position of the fairing geometry, $r_{f a i r_{m a x}}$, is determined by first locating the axial location of plane $2, z_{2}$, using $\tilde{z}_{2}$ and the equation

$$
z_{2}=z_{3}\left(1-\tilde{z}_{2}\right)
$$

where $z_{3}$ is the plane 3 location and corresponds to the rocket nozzle exhaust plane. The

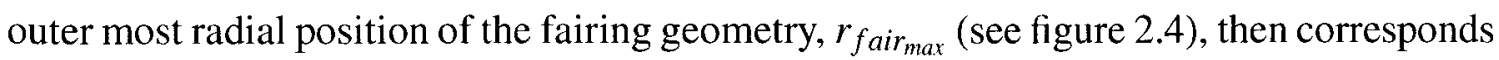
to the radius of the inner rocket nozzle contour at $z_{2}$. The chord length of the fairing at $r_{\text {fair }}$ max is defined as

$$
c_{\text {fair }}=z_{3} \tilde{c}_{\text {faix }} \text { max }_{\text {max }}
$$

Finally the profile's maximum thickness ratio at the outer most radial position is calculated 


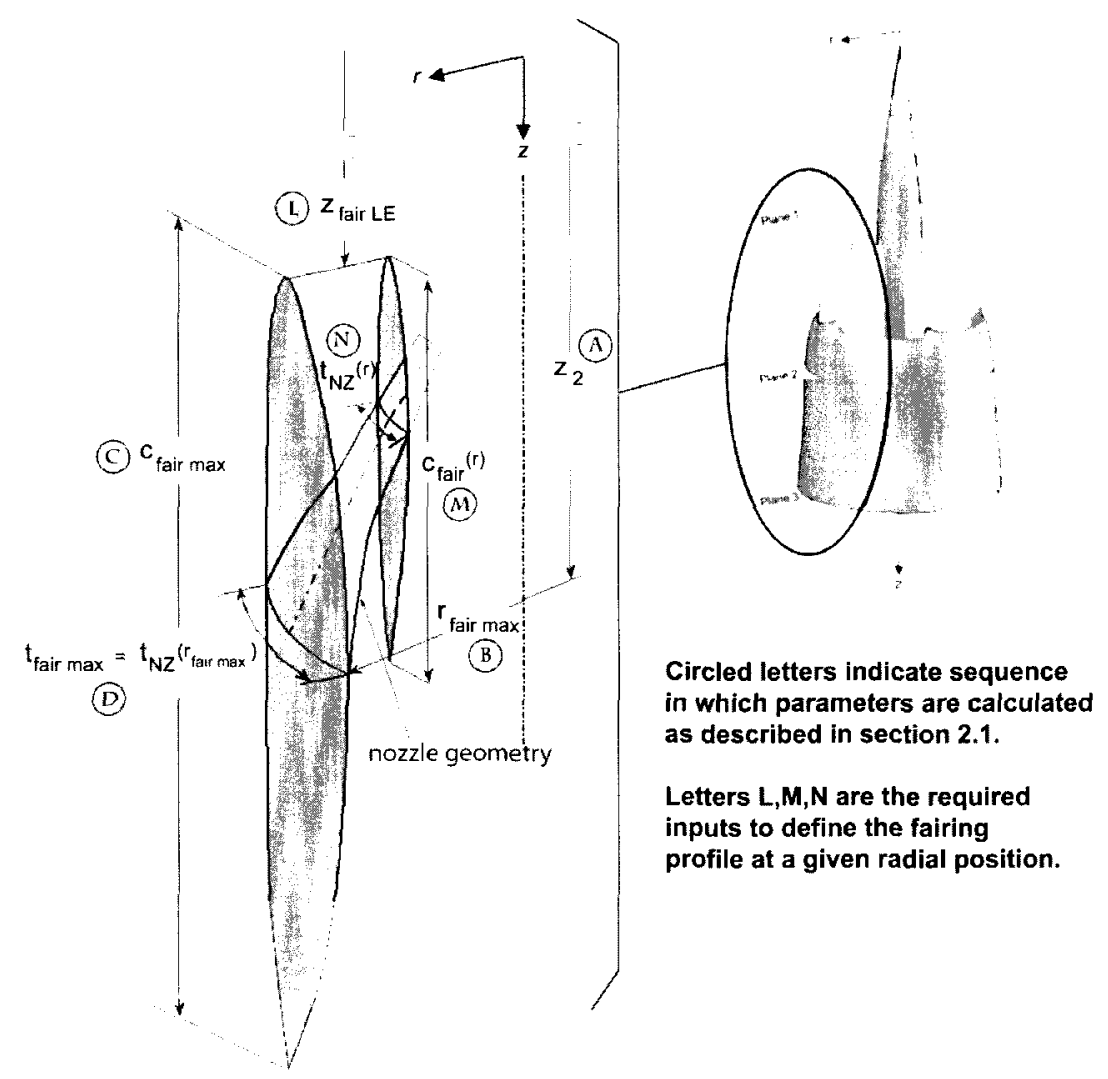

Figure 2.4: Fairing profile definitions.

using,

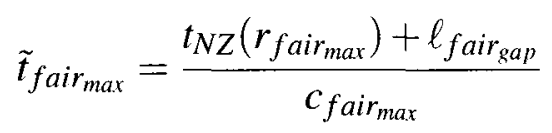

where $\ell_{\text {fair gap }}$, a secondary input parameter, is the minimum desired gap between the nozzle surface and fairing surface, and $t_{N Z}$ is the thickness of the nozzle at the given radial position and hence is a pre-defined input. The axial location of the maximum thickness of the fairing profile is aligned to be coincident with the nozzle geometry, as shown in figure 2.4. Positioning the fairing profile in this manner ensures that the fairing geometry does not significantly reduce the projected area in the x-y plane through the intake. The axial location of the leading edge, $z_{\text {fair }}$, of the outer most radial fairing profile is then used as the leading edge location for the fairing profiles at all remaining radial positions. 
With the leading edge location defined and $t_{N Z}$ known at all radial locations from the nozzle contour, the chord length and maximum thickness ratio of the fairing profiles at any radial location can be defined. Recalling that the location of maximum thickness of the base fairing profile is 0.4 as a fraction of chord length for the NACA 0020-34 profile, the chord length at a given radial position is defined as

$$
c_{\text {fair }}(r)=\frac{z_{N Z}(r)-z_{\text {fair }}}{0.4}
$$

where $z_{N Z}(r)$ is the axial location of the nozzle contour at a given radial position. The local maximum thickness ratio at a given radial position is defined as

$$
\tilde{t}_{\text {fair }}(r)=\frac{t_{N Z}(r)+\ell_{\text {fair }_{\text {gap }}}}{c_{\text {fair }}(r)}
$$

This local thickness ratio is used to scale the base fairing profile such that the fairing profile encloses the nozzle geometry with the desired clearance.

At this point all three parameters required to define the fairing profile at any given radial position, $\left(z_{\text {fair }_{L E}}, c_{\text {fair }}(r)\right.$, and $\left.\tilde{t}_{\text {fair }}(r)\right)$ are passed to the centre body geometry phase in order to accurately define the flow areas at plane 2 and plane 3.

\subsection{Centre Body Geometry}

The axisymmetric centre body is divided into the four regions shown in figure 2.5 and is defined using the known nozzle and fairing geometry along with the input parameters $\sigma$, $A R, \tilde{t}_{3}$, and $\tilde{r}_{C B_{1}}$. Centre body region $\mathrm{A}$ is the portion of the centre body between the nose and plane 1. Centre body region $B$ lies between planes 1 and 2 . Centre body region $C$ is the duct portion of the air intake geometry and is defined between plane 2 and plane 3 . Center body region $\mathrm{D}$ extends downstream from plane 3 to a length, $l_{D}$, which is a function 
of $r_{C B_{3}}$ and is defined as a secondary input. Cubic hermite curves are used to define the center body contour in each region. The hermite curves are constrained at the boundaries of each centre body region by specifying both the radial location and the slope of the surface at the boundary points. The centre body radius at plane $3, r_{C B_{3}}$, is defined using

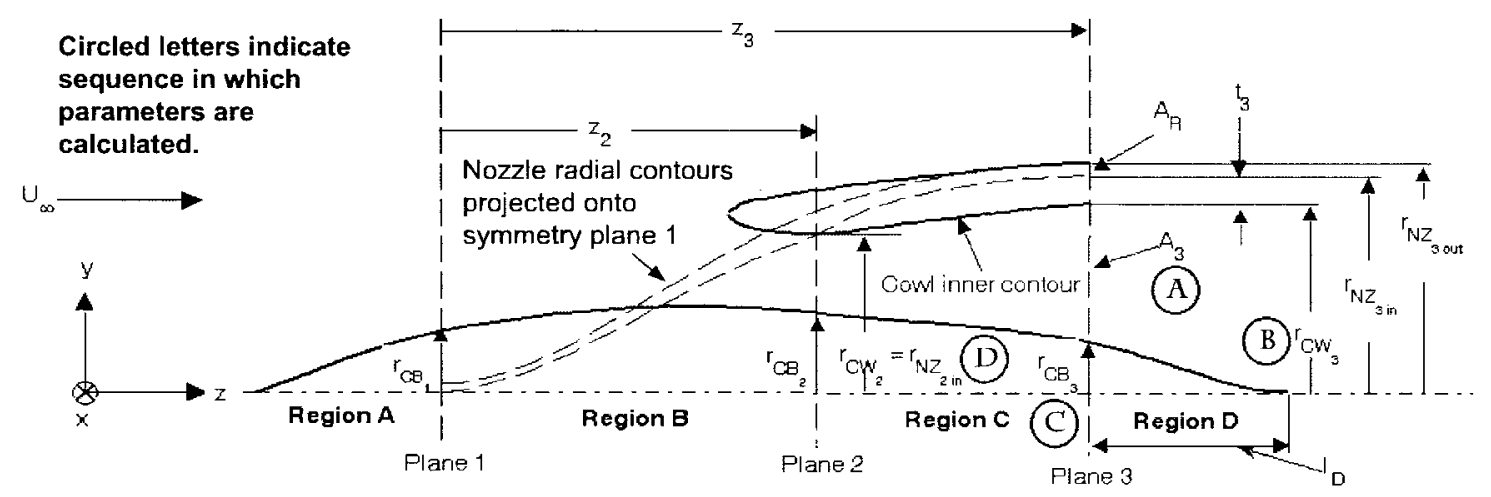

Figure 2.5: Cross section view along symmetry plane 1 of proposed air intake geometry.

the parameters $\sigma$ and $\tilde{t}_{3}$, in combination with the rocket nozzle geometry. First, using the specified $\sigma$ and the known rocket exhaust flow area, $A_{R}$, the flow area at plane 3 is given by

$$
A_{3}=A_{R}\left(\frac{1}{\sigma}-1\right)
$$

The radius of the cowl inner contour at plane 3 , identified in figure 2.5 , is determined using $\tilde{t}_{3}$ and the equation

$$
r_{C W_{3}}=r_{N Z_{3 i n}}\left(1-\tilde{t}_{3}\right)
$$

Knowing the maximum radius at plane 3 , the following procedure is used to determine the value of $r_{C B_{3}}$ required to obtain the flow area $A_{3}$. The presence of the fairing geometry at plane 3 is accounted for by using the fairing geometry information and taking an $\mathrm{x}$ $y$ plane cross section of plane 3 as shown in figure 2.6. The plane is first divided into 


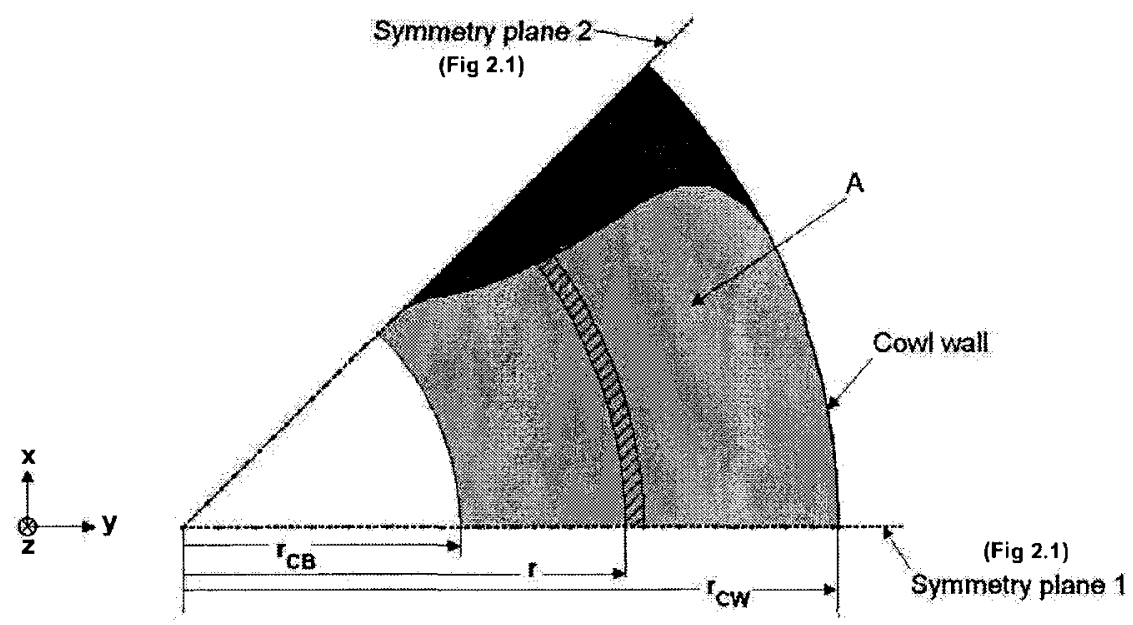

Figure 2.6: x-y cross section view of outflow plane.

radial segments. The flow area for different values of $r_{C B}$, starting from the cowl wall and moving towards the centre line is found. An example plot of the relationship between flow area and centre body radius is shown in figure 2.7. A cubic spline fit is applied to the flow area centre body radius relationship, and then knowing the target flow area, $A_{3}$, a Newton-Rhapson method is used to solve for $r_{C B_{3}}$ such that the target flow area is met.

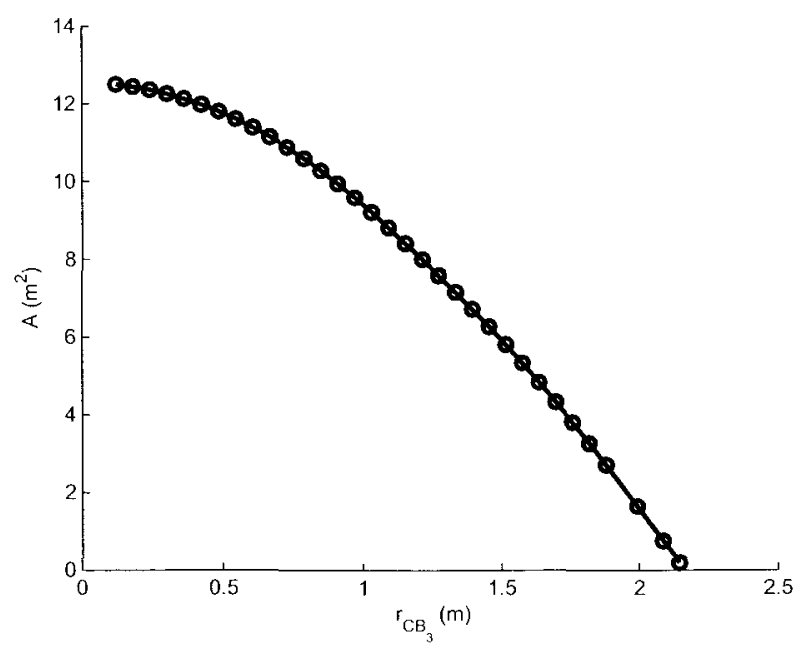

Figure 2.7: Relationship between flow area and $r_{C B_{3}}$.

The centre body radius at plane $2, r_{C B_{2}}$, is defined using the input parameter $A R$ as well 
as $z_{2}$ (found previously, see Eq. (2.1)) and the inner radial contour of the nozzle. First, the plane 2 flow area, $A_{2}$ is found as

$$
A_{2}=A_{3} A R
$$

Then, $r_{C B_{2}}$ is solved for in a similar manner to $r_{C B}$ with the the inner nozzle radius at plane $2, r_{N Z_{2} \text { in }}$, acting as the cowl wall and using $A_{2}$ as the target flow area. Finally, the center body radius at plane $1, r_{C B_{1}}$, is defined as a multiple of the nozzle radius at plane 1 using the input parameter $\tilde{r}_{C B_{1}}$. The slopes of the center body curve at the region boundaries must be defined to complete the center body curve definition. The slope of the center body curve at the end of region $\mathrm{D}$ is set to $1^{\circ}$ above horizontal since in the absence of viscous effects it is desirable for the flow to leave the center body as straight as possible. By default the geometry creation method calculates an average slope to ensure smooth transitions between center body regions. The slopes at each plane are calculated as follows

$$
\begin{gathered}
\left(\frac{\Delta r}{\Delta z}\right)_{1}=\left(\frac{r_{C B_{2}}-r_{C B_{1}}}{z_{2}-z_{1}}\right)+\left(\frac{r_{C B_{1}}}{z_{1}-z_{C B_{\text {nose }}}}\right) \\
\left(\frac{\Delta r}{\Delta z}\right)_{2}=\left[\left(\frac{r_{C B_{2}}-r_{C B_{1}}}{z_{2}-z_{1}}\right)+\left(\frac{r_{C B_{3}}-r_{C B_{2}}}{z_{3}-z_{2}}\right)\right] / 2 \\
\left(\frac{\Delta r}{\Delta z}\right)_{3}=\left[\left(\frac{r_{C B_{3}}-r_{C B_{2}}}{z_{3}-z_{2}}\right)+\left(\frac{-r_{C B_{3}}}{l_{D}}\right)\right] / 2
\end{gathered}
$$

\subsection{Cowl Geometry}

The cowl geometry is separated into two regions; the cowl lip and the duct region between planes 2 and 3. The duct region is defined with an inner and outer radial contour.

The cowl lip is defined using an elliptical profile for the inner and outer lip surfaces. An elliptical profile has been chosen as opposed to a semi-circular profile as elliptical profiles have been shown to perform well [18]. These elliptical profiles are defined based 
on the cowl geometry, the lip contraction ratio, $C R$, which is a primary input parameter, and the secondary input parameter $l_{a} / l_{b}$, which is set to a value of $l_{a} / l_{b}=4.5$ by default and is the ratio between the semi-major and semi-minor ellipse lengths for the inner lip surface (Fig 2.8).

When air intakes operate at large flow ratios (i.e. where the cross-sectional area of the incoming flow stream tube is much larger than the initial duct flow area) the dividing streamline between the intake flow and the external flow stream tubes will tend to stagnate on the outer surface of the cowl lip. In this situation the flow is forced to turn around the cowl lip which results in large peak velocities at the lip leading edge. The flow is then rapidly decelerated downstream of the lip leading edge as it enters the duct and thus experiences large adverse pressure gradients which can cause flow separation. The lip contraction ratio, $C R$, has been shown [18] to be the parameter which has the most significant control over the magnitude of the peak flow velocities along the cowl lip and therefore controls at what flow ratios boundary layer separation along the cowl lip occurs. The $C R$ is defined below as

$$
C R=\frac{A_{C W_{L E}}}{A_{2}}
$$

where $A_{2}$ is the duct throat area and $A_{C W_{L E}}$ is the flow area at the leading edge of the cowl lip. As $C R$ increases, peak lip velocities decrease and losses due to flow separation along the cowl lip decrease. In addition, the ratio $l_{a} / l_{b}$ affects the peak flow velocities with flow velocities decreasing with decreasing $l_{a} / l_{b}$. The cowl lip elliptical profiles can be fully defined by specifying their centre point and their semi-major and semi-minor lengths, shown in figure 2.8 .

The inner lip surface is defined by specifying the location of points $\mathrm{A}$ and $\mathrm{C}$. The axial location of point $\mathrm{A}$ is located at plane 2 and at a radius equal to the inner rocket nozzle radius at plane 2 . The required flow area, $A_{C W_{L E}}$, at the lip leading edge is calculated 


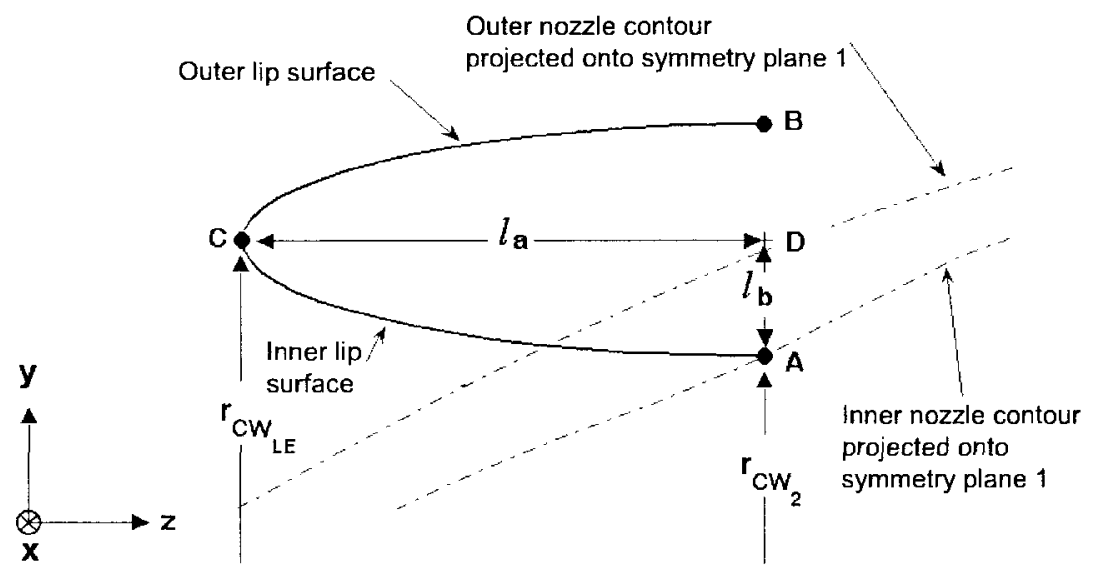

Figure 2.8: Cowl lip geometry definitions

using the given $C R$ and Eq. 2.12. Point $C$ is then located using $C R, l_{a} / l_{b}$, and the centre body curvature. Since the $\mathrm{z}$ and $\mathrm{y}$ coordinates of point $\mathrm{C}$ are initially unknown, in order to enforce both the desired $C R$ and $l_{a} / l_{b}$, an iterative method is used. Initially, the $\mathrm{y}$ coordinate of point $\mathrm{C}$ is found, assuming a centre body radius of $r_{\mathrm{CB}_{2}}$, by finding the cowl lip radius for which the flow area is equal to $A_{C W_{L E}}$, which ensures the desired $C R$ is met. Next, the y coordinate of point $C$ is used along with $r_{\mathrm{CW}_{2}}$ to calculate the value of $l_{b}$, shown in figure 2.8 , which is then used along with the desired $l_{a} / l_{b}$ to find the $\mathrm{z}$ coordinate of point $\mathrm{C}$. However, because the centre body at the initial $\mathrm{z}$ coordinate of point $\mathrm{C}$ is not necessarily equal to $r_{C B_{2}}$, the actual flow area at point $\mathrm{C}$ may not equal $A_{C W_{L E}}$. Therefore, the desired value of $C R$ will not be met. The procedure used to solve for a point $C$ location such that both $C R$ and $l_{a} / l_{b}$ are met begins by solving for the $\mathrm{y}$ and $\mathrm{z}$ coordinates of point $\mathrm{C}$ as previously described. An updated y position of point $\mathrm{C}$ is then calculated to obtain the desired $A_{C W_{L E}}$, which is then used to calculate the value of $l_{b}$, which is then used along with the desired $l_{a} / l_{b}$ to update the $\mathrm{z}$ coordinate of point $\mathrm{C}$. This update procedure is repeated until the desired values of $C R$ and $l_{a} / l_{b}$ are met. The centre point, $\mathrm{D}$, of the ellipse is then determined based on points $\mathrm{A}$ and $\mathrm{C}$ (it is not necessarily coincident with the outer rocket nozzle contour). 
The initial inner and outer lip surfaces are defined by an ellipse with centre point $D$ and the ratio $l_{a} / l_{b}$. Since the lip surfaces and cowl surfaces should blend together smoothly at plane 2, inner and outer blend regions, highlighted in figure 2.9, are added between the two surfaces. The outer blend region begins at a point along the initial outer lip surface and ends at a point along the outer nozzle surface between plane 2 and plane 3 . These start and end locations are selected based on local surface slope.

The start point is located along the initial outer lip surface at the location where the surface slope equals the average slope along the outer nozzle radial contour downstream of plane 2. The location of the outer nozzle radial contour, projected onto symmetry plane 1 , is shown in figure 2.9. The calculation of this average slope is simply the average between the slope of the outer nozzle radial contour at plane 2 and at plane 3 . The end point of the outer blend region is located on the nozzle outer radial contour where the surface slope is $60 \%$ of the surface slope along the lip at the start point. This ratio between the surface slopes at the start and end points of the blend region has been determined through trial and error and has been shown to produce smooth blend regions over a large range of cowl shapes. With the start and end locations of the outer blend region known, along with the surface slopes at these points, the outer blend region surface is defined using a hermite curve. The outer cowl surface downstream of the outer blend region is set to be coincident with the existing rocket nozzle outer contour.

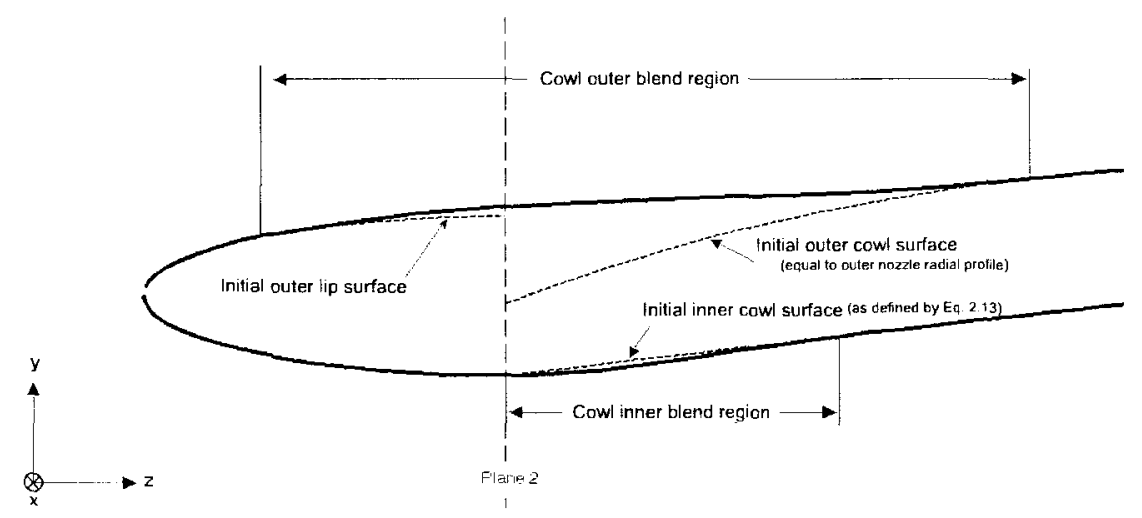

Figure 2.9: Cowl outer and inner blend regions 
The initial cowl inner contour is determined based on the centre body contour in region $\mathrm{C}$ and the prescribed axisymmetric flow area distribution through the duct, $A_{2-3}$. This flow area distribution can currently be specified to vary linearly or quadratically through region $\mathrm{C}$; however, any distribution can be used. The duct flow area distribution varies from $A_{a x i_{2}}$ at plane 2 to $A_{a x i_{3}}$ at plane 3, where $A_{a x i}$ is simply the axisymmetric flow area obtained using the radial values of the centre body and cowl surfaces at a given $z$ location. The axisymmetric flow area therefore does not account for the presence of the fairing geometry. The cowl interior contour is expressed as

$$
r_{C W_{i n}}(z)=\left(\frac{A_{a x i}(z)}{\pi}+r_{C B}(z)^{2}\right)^{\frac{1}{2}}
$$

where the flow area as a function of $\mathrm{z}, A_{\text {axi }}(z)$ is defined as

$$
\begin{aligned}
& A_{2-3}=\text { Linear }: \quad A_{a x i}(z)=\frac{A_{a x i_{3}}-A_{a x i_{2}}}{z_{3}-z_{2}}\left(z-z_{2}\right)+A_{a x i_{2}} \\
& A_{2-3}=\text { Quadratic: } \quad A_{a x i}(z)=\frac{A_{a x i_{2}}-A_{a x i_{3}}}{\left(z_{3}-z_{2}\right)^{2}}\left(z-z_{2}\right)^{2}-\frac{2\left(A_{a x i_{2}}-A_{a x i_{3}}\right)}{\left(z_{3}-z_{2}\right)}\left(z-z_{2}\right)+A_{a x i_{2}}
\end{aligned}
$$

The start point of the inner blend region is located along the inner lip surface at plane 2 , and the end point is located 1 lip length $\left(l_{a}\right)$ downstream of plane 2 . Using the location of the start and end points and the local surface slopes, the inner blend region is defined using a hermite curve.

\subsection{D Geometry Creation}

In order to run 3D computational fluid dynamics (CFD) simulations as well as build scale models of the air intake geometry, a 3D model of the air intake geometry must be generated. The 3D air intake bodies are created by generating point surfaces, which are then 
imported into ANSYS ${ }^{\circledR}$ ICEM 11.0 and stitched together to form a solid body. The centre body and cowl point surfaces are generated by sweeping the 2D profiles by an angle $\chi$, which is the angle between the two symmetry planes. The fairing point surfaces are generated by determining the fairing profiles at several radial positions along the nozzle and connecting the profiles to form a 3D surface. At the interfaces between intake bodies, surfaces are blended by creating fillet surfaces, shown in figure 2.10 . The fillet radii of the various intake surface interfaces are included as secondary input parameters and are referenced to the rocket nozzle width between the inner and outer nozzle radial contours at plane 2. The fillet geometry is created according to the fixed radius fillet algorithm described by Yamashina et al. [38]

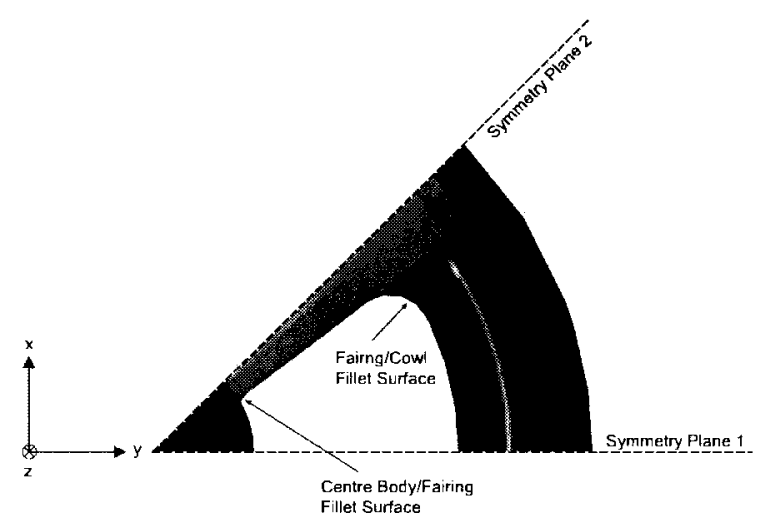

Figure 2.10: Top view of 3D geometry, highlighting fillet surfaces.

\subsection{Geometry Results}

A geometry input parameter study has been conducted to determine the influence of each parameter on the air intake geometry as well as to identify any constraints on each parameter. The 8 input parameters along with their typical values are listed in table 2.2 and the results from the parameter study are shown in figure 2.12 and figure 2.13 .

Changes in $\tilde{z}_{2}$, shown in figure 2.12 (a), affect the duct length as well as the centre body 
Table 2.2: Primary Input Parameter Summary

\begin{tabular}{rr} 
Parameter & Typical Values \\
\hline$\tilde{z}_{2}$ & $0.3-0.5$ \\
$\tilde{c}_{\text {fair }}$ max & $0.9-1.3$ \\
$\sigma$ & $0.05-0.3$ \\
$\tilde{t}_{3}$ & $0.05-0.2$ \\
$A R$ & $0.4-0.8$ \\
$\tilde{r}_{C B_{1}}$ & $2-8$ \\
$C R$ & $1.05-1.4$ \\
$A_{2-3}(z)$ & Linear - Quadratic
\end{tabular}

radius at plane 2. As $\tilde{z}_{2}$ increases, the location of plane 2 moves upstream. Because the cowl radius at plane 2 is set to be coincident with the inner rocket nozzle contour and the rocket nozzle radius decreases as one moves upstream, $r_{\mathrm{CW}_{2}}$ decreases as $\tilde{z}_{2}$ increases. As $r_{\mathrm{CW}_{2}}$ decreases, $r_{\mathrm{CB}_{2}}$ must also decrease so that the desired plane 2 flow area can be maintained. Since the maximum possible flow area at plane 2 occurs when $r_{C B_{2}}=0$ and $r_{C W_{2}}$ decreases as $\tilde{z}_{2}$ increases, for a given $A R$ there is maximum value of $\tilde{z}_{2}$. Due to structural constraints, $r_{C_{2}}$ cannot be zero. Instead, a minimum radius is specified as a secondary input parameter. In the event the specified $A R$ is not possible for a given $\tilde{z}_{2}$ and minimum value of $r_{C B_{2}}$, the value of $A R$ is decreased such that $r_{C B_{2}}$ is equal to the minimum radius.

Changes in $\tilde{c}_{\text {fair }}$, shown in figure 2.12(b), affect the centre body radius at both plane 2 and plane 3 . As $\tilde{c}_{\text {fair }}$ max decreases, chord length decreases and the fairing geometry volume within the duct is reduced. Consequently, the center body radius increases to maintain the desired flow area. Because the fairing leading edge location is determined based on the nozzle geometry at plane 2 and $c_{\text {fair } r_{\max }}$, which is a function of $\tilde{c}_{\text {fair }}$ max , if $\tilde{c}_{\text {fair }_{\text {nut }}}$ is too small, a portion of the nozzle geometry may be left exposed, as illustrated in 


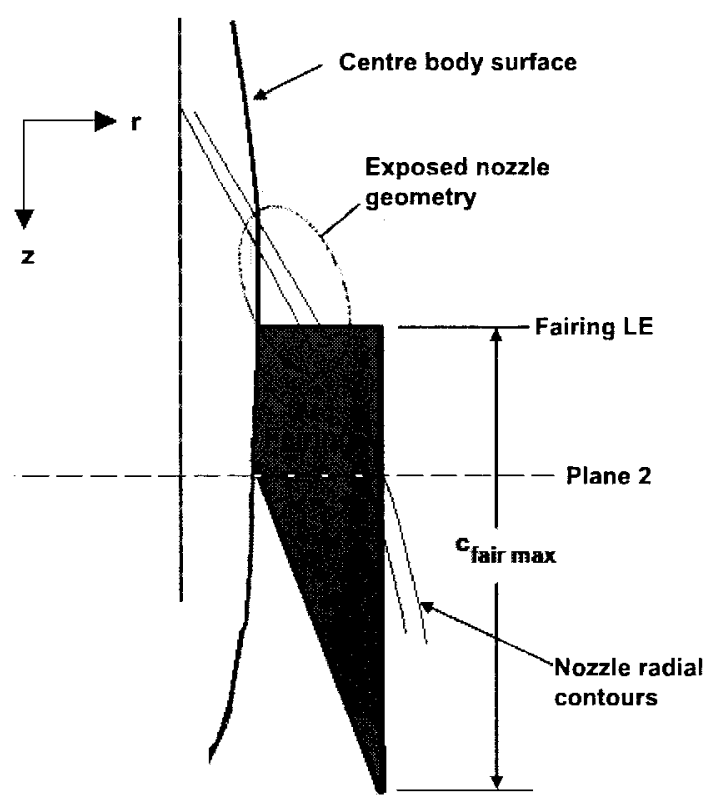

Figure 2.11: Side view of fairing showing a situation where the fairing does not completely cover the nozzle geometry.

figure 2.11. Therefore the minimum value of $\tilde{c}_{f a i r_{\text {max }}}$ is set such that the nozzle geometry is completely enclosed by the fairing.

Changes in $\sigma$, shown in figure 2.12(c), significantly affect the centre body radius at both plane 2 and plane 3, with the centre body radius increasing with increasing $\sigma$. Recalling Eq. 2.6 and noting that $A_{R}$ is fixed for a given rocket nozzle geometry and $\sigma$ is constrained to the range $0<\sigma<1$, as $\sigma$ increases, $A_{3}$ will decrease. In order for $A_{3}$ to decrease, the centre body radius at plane 3 must increase and since $A R$ is being held constant, the center body radius at plane 2 also increases. In addition, as $r_{C B_{3}}$ increases with increasing $\sigma$, the centre body tail length, $l_{D}$, increases because it is set as a fixed multiple of $r_{C B_{3}}$. The changes in the centre body geometry caused by changes in $\sigma$ also affect the cowl lip geometry since the lip geometry is a function of the centre body curve. The minimum value of $\sigma$ is limited by the nozzle geometry, where the minimum value of $\sigma$, and thus the maximum value of $A_{3}$ is achieved when $\tilde{t}_{3}=0$ and $r_{C B_{3}}=0$. If the specified value of $\sigma$ is not possible given the remaining input parameters, the value of $\sigma$ is increased until 


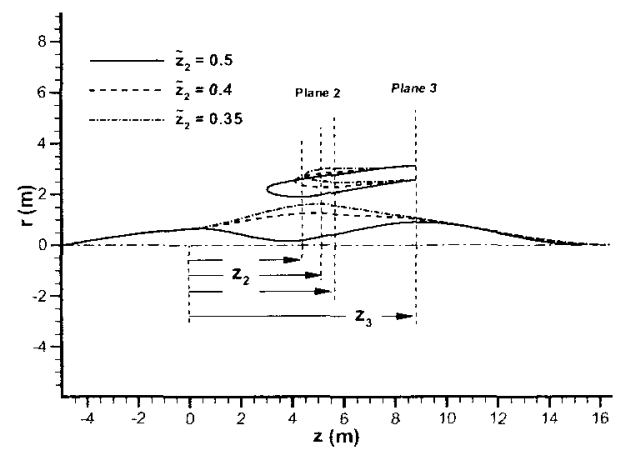

(a) Influence of $\tilde{z}_{2}$.

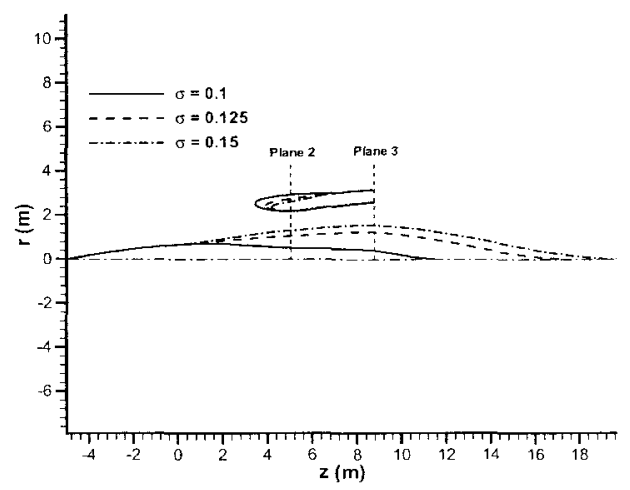

(c) Influence of $\sigma$.

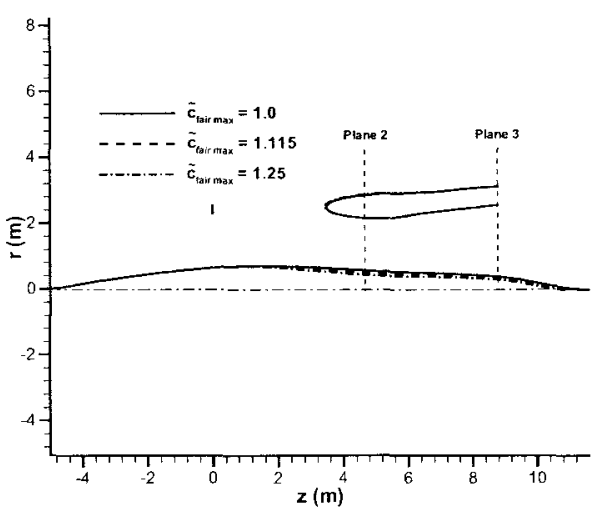

(b) Influence of $\tilde{c}_{\text {fair }}$ max .

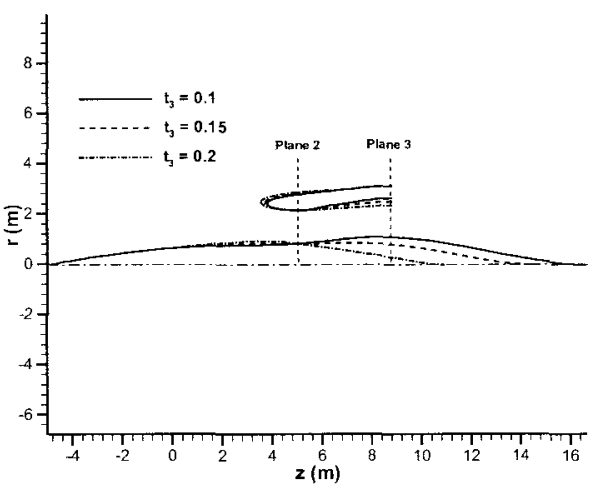

(d) Influence of $\tilde{t}_{3}$.

Figure 2.12: Results from first four geometry input parameter studies viewed along symmetry plane 1 .

there are no geometric inconsistencies.

Changes in $\tilde{t}_{3}$, shown in figure $2.12(\mathrm{~d})$, significantly affect the center body radius and cowl radius at plane 3 . As $\tilde{t}_{3}$ increases, $r_{C W_{3}}$ decreases according to Eqn. 2.7 and thus $r_{C B_{3}}$ must decrease in order to maintain the required $A_{3}$. As $r_{C W_{3}}$ decreases, the centre body tail length, $l_{D}$, also decreases. The change in $r_{C B_{3}}$ does not change the value of $r_{C B_{2}}$, however; it can change the surface slope at plane 2 according to Eqn. 2.10 and thus alters the centre body shape in regions B and C (Fig 2.5). The change in the centre body shape in region B 
alters the cowl lip geometry, and the change in region $\mathrm{C}$ alters the inner cowl surface. The value of $\tilde{t}_{3}$ is constrained to the range $0<\tilde{t}_{3}<1$.

Changes in $A R$, shown in figure 2.13(a), affect the center body radius at plane 2 but do not significantly influence the geometry at plane 3 . Recalling that for fixed $\sigma, A_{3}$ is fixed, as $A R$ increases, $A_{2}$ must increase, which requires a decrease in $r_{C B_{2}}$. Changes in $A R$ also have a minor influence on the cowl lip geometry and the inner cowl surface. The cowl lip leading edge radius changes due to the changing centre body curve, which in turn affects the length of the lip since the lip $\frac{l_{a}}{l_{b}}$ ratio is fixed. The inner cowl surface is a function of both the prescribed axisymmetric area distribution and the centre body curve. Limitations on the value of $A R$ are coupled to $\tilde{z}_{2}$ and have been discussed in the previous paragraph.

Changes in $\tilde{r}_{C B_{1}}$, shown in figure $2.13(\mathrm{~b})$, affect the centre body radius at plane 1 , the location of the centre body nose, and the centre body surface upstream of plane 2 . Because changes in $\tilde{r}_{C B_{1}}$ affect the centre body in region $\mathrm{B}$, the cowl lip geometry is slightly affected. The ratio between $r_{C B_{1}}$ and the length of the centre body upstream of plane 1 is set as a secondary input parameter. The only constraint placed $\tilde{r}_{C B_{1}}$ is that it must have a value greater than 1 .

Changes in $C R$, shown in figure 2.13(c), affect all three cowl surfaces; the cowl lip, the inner cowl surface, and the outer cowl surface. Recalling Eqn. 2.12, for a fixed $A_{2}$, as $C R$ increases, $A_{C W_{L E}}$ must increase and therefore the radius of the lip leading edge $\left(r_{C W_{L E}}\right)$ increases. As $r_{C W_{L E}}$ increases, the lip ellipse semi-minor length increases. Since the lip ellipse ratio $\frac{l_{a}}{l_{b}}$ is fixed, an increasing semi-minor length causes an increase in the ellipse semi-major length and thus increases the lip length. The changes in cowl lip height and length affect the blend regions along the inner and outer cowl surfaces in the manner discussed in Section 2.3. It has been found that values of $C R$ between 1-2 work well for Mach numbers between $0-0.9$, and therefore $C R$ is constrained to this range.

Changes in $A_{2-3}$, shown in figure $2.13(\mathrm{~d})$, only affect the cowl radial contour since 


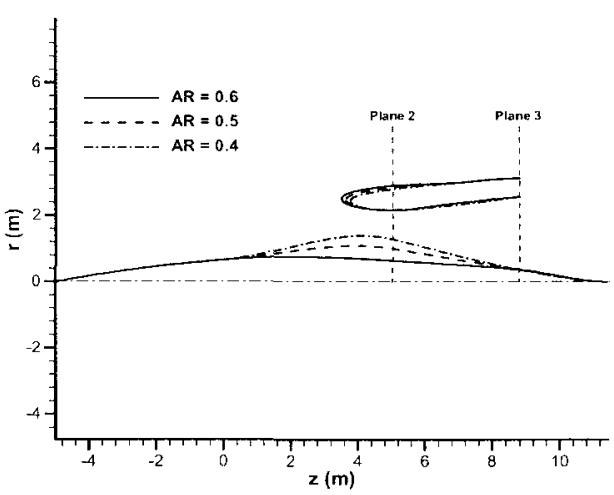

(a) Influence of $A R$.

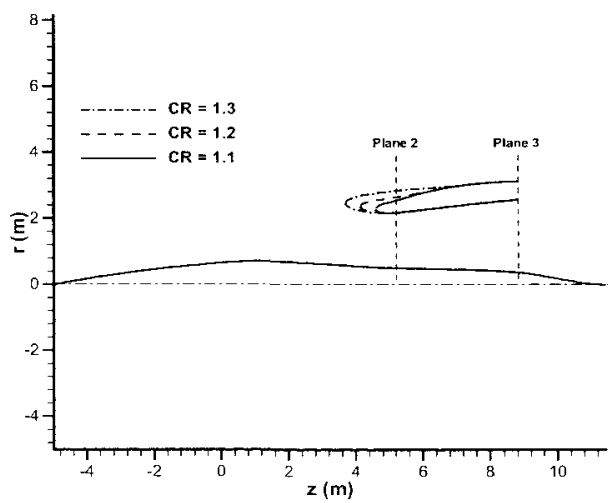

(c) Influence of $C R$.

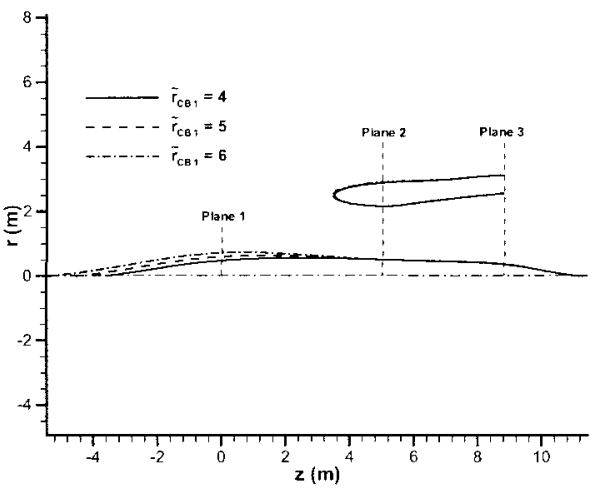

(b) Influence of $\tilde{r}_{C B_{1}}$.

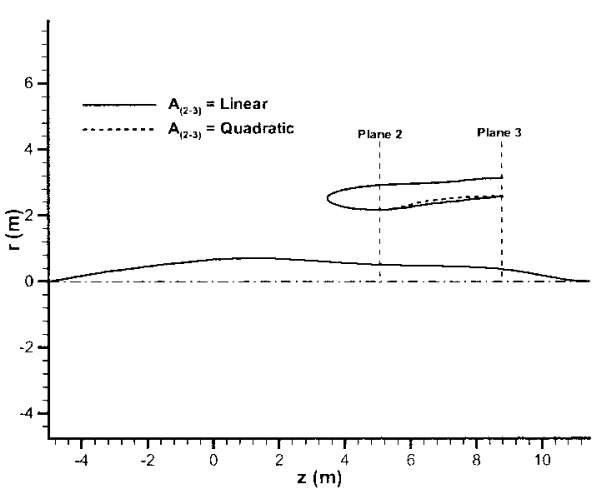

(d) Influence of $A_{2-3}$.

Figure 2.13: Results from remaining four geometry input parameter studies viewed along symmetry plane 1 .

$A_{2-3}$ is only used to define this surface (see Eq. (2.13)). 


\section{Chapter 3}

\section{Air Intake Design Method: Flow Analysis}

The second step in the air intake design method is to predict the performance of the air intake geometry while operating at certain flight conditions. The air intake geometry's performance is measured in terms of air mass flow rate, $\dot{m}$, which is defined as

$$
\dot{m}=\left(\overline{\rho_{3}} A_{3} \bar{U}_{z_{3}}\right)^{(v i s c)}
$$

where the superscript (visc) indicates the corrected values of $\overline{\rho_{3}}, A_{3}, \bar{U}_{z_{3}}$, due to viscous

affects. The effective flow area at plane $3, A_{3}^{(v i s c)}$, is affected by boundary layer growth and boundary layer separation along the geometry surfaces. The effective density and axial velocity at plane $3, \rho_{3}^{(v i s c)}$ and $U_{z_{3}}^{(v i s c)}$ are affected by viscous flow losses.

The flow analysis method consists of an inviscid flow solver and a viscous flow solver. The invisicd flow solver calculates the surface velocities along the intake surfaces using inviscid, incompressible panel methods $[23,25]$ along with a compressibility correction [27]. The viscous solver calculates the boundary layer development along the intake surfaces using these surface velocities and a turbulent compressible integral boundary layer method [20]. 


\subsection{Flow Condition Input Parameters}

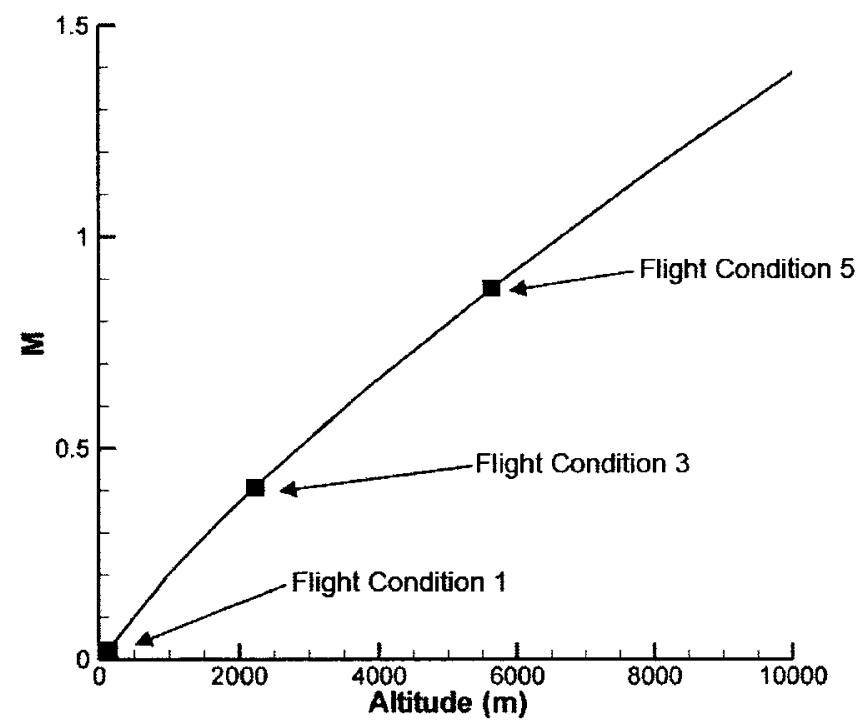

Figure 3.1: Ariane 4 flight profile. Derived from Turner [39]

The flow analysis requires 3 flow condition input parameters, $M_{\infty}, h_{A l t}$, and $p_{3}$, to specify the air intake operating conditions. These parameters specify at what point in the RBCC vehicles flight profile, (an Ariane 4 profile is shown in figure 3.1), the designer wishes to evaluate the air intake performance. The input parameters $M_{\infty}$ and $h_{A l t}$ are used to define the flight velocity and altitude of the RBCC vehicle respectively. Using $h_{A l t}$ and a NASA atmospheric model [40], the local static flow conditions can be calculated, and using $M_{\infty}$, the total flow conditions can be calculated.

The input parameter $p_{3}$ allows the user to define the static pressure at plane 3 , which is determined based on the flow conditions at the intake to the rocket-ejector. The average compressible flow velocity at plane 3 , which is required for the inviscid flow solver, is calculated using $p_{3}, P_{0}$, and the isentropic flow relations. 


\subsection{Inviscid Flow Solver}

The purpose of the inviscid flow solver is to predict the velocity distribution and thus the pressure distribution along all three intake bodies. The inviscid flow solver used in this flow analysis consists of two components: a potential flow panel method, and a flow field combination procedure. These two components are required because when modeling intake flows, the desired mass flow rate through the air intake must be enforced while also meeting the required freestream flow velocity. To enforce these two conditions, a potential flow panel method is used to calculate two different inviscid flow fields around the air intake geometry. Then the combination procedure is applied to these two inviscid flow fields such that the desired intake mass flow rate and freestream velocity are met. Two types of panel method are used: an axisymmetric panel method by Hess [25] for the flow over the centre body and cowl surfaces, and a 2D panel method by Hess [23] for flow over the fairing surfaces.

\subsubsection{Potential Flow Panel Methods}

In potential flow panel methods, incompressible, irrotational flow around bodies of arbitrary shape can be simulated by breaking up the body surface into $N$ discrete panels which contain combinations of potential flow elements such as sources and vortices. Each surface panel contains a control point at which a normal velocity boundary condition is enforced. Surface panels are identified with the index $j$ where $j=1,2, \ldots, N$ and surface panel control points are identified with the index $i$ where $i=1,2, \ldots, N$. The strengths of the potential flow elements at the control point of each surface panel are solved such that the local flow velocity normal to each surface panel control point is zero so that

$$
\vec{U}_{\infty} \cdot \hat{n}_{i}+\vec{u}_{i} \cdot \hat{n}_{i}=U_{\perp_{\perp_{i}}}+u_{\perp_{i}}=0
$$


where $\vec{U}_{\infty}$ is the freestream velocity vector and is constant throughout the flow domain, $\vec{u}_{i}$ is the disturbance velocity vector at panel control point $i$ due to the potential flow elements, and $\hat{n}_{i}$ is the unit normal vector at panel control point $i$. The subscript $\perp$ is used to denote the component normal to a panel. Enforcing the zero normal velocity condition at each surface panel ensures that the dividing flow streamlines fit the shape of the arbitrary body.

Both panel methods used in this work use a combination of sources and vortices on the surface panels. The source strength on each panel is unique but the vortex strength on each panel is identical. Recalling that the disturbance velocity vector, $\vec{u}_{i}$, is a function of these potential flow elements, the disturbance velocity at panel control point $i$ can be written as

$$
\vec{u}_{i}=\sum_{j=1}^{N} \vec{V}_{i j} \lambda_{j}+\sum_{j=1}^{N} \vec{V}_{i j}^{(\text {vort })} \Gamma
$$

where $\vec{V}_{i j}$ is the source influence coefficient vector of the $j$-th panel on the $i$-th control point, $\lambda_{j}$ is the source strength at the $j$-th panel, $\vec{V}_{i j}^{(v o r t)}$ is the vortex influence coefficient vector of the $j$-th panel on the $i$-th control point, and $\Gamma$ is the vortex strength. The source and vortex influence coefficient vectors are functions of the panel geometry and are therefore known once the panel geometry is defined. Letting $V_{\perp_{i j}}$ and $V_{\perp_{i j}}^{(v o r)}$ represent the normal component of the source and vortex influence coefficient vectors respectively, and remembering the zero normal velocity boundary condition, Eqn 3.2 and Eqn 3.3 can be combined to give

$$
U_{\perp_{i}}=U_{\infty_{L_{i}}}+\sum_{j=1}^{N} V_{\perp_{i j}} \lambda_{j}+\sum_{j=1}^{N} V_{\perp_{i j}}^{(v o r t)} \Gamma=0
$$

Equation 3.4 can then be rearranged to the form

$$
\sum_{j=1}^{N} V_{\perp_{i j}} \lambda_{j}+\sum_{j=1}^{N} V_{\perp_{i j}}^{(v o r t)} \Gamma=-U_{\infty_{\perp_{i}}}
$$

Equation 3.5 forms a $(N) \times(N+1)$ linear system of equations with $(N+1)$ unknowns 
and forms the base equation for the potential flow panel method. The $(N+1)$ unknowns are the $N$ source strengths, $\lambda_{j}$ and the vortex strength, $\Gamma$. However, because there are only $(N)$ surface panels, the velocity influence coefficient matrix is an $N \times(N+1)$ matrix. Therefore, in order to solve for the $(N+1)$ unknowns, an additional boundary condition is required. The type of boundary condition used depends on the flow situation being simulated and is discussed in further detail in section 3.2.2 .

Once the source and vortex strengths are known, the velocity along the intake surfaces or anywhere within the flow domain can be calculated. The velocity tangent to surface panel $i$, the surface velocity, $U_{\|_{i}}$, can be calculated with the equation

$$
U_{\|_{i}}=U_{\infty_{\|_{i}}}+\sum_{j=1}^{N} V_{\|_{i j}} \lambda_{j}+\sum_{j=1}^{N} V_{\|_{i j}}^{(\text {vort })} \Gamma
$$

where the subscript $\|$ is used to denote the component tangent to a surface panel. The velocity vector at point $\wp$ in the flow domain can be calculated with the equation

$$
\vec{U}_{\wp}=\vec{U}_{\infty}+\sum_{j=1}^{N} \vec{V}_{\wp j} \lambda_{j}+\sum_{j=1}^{N} \vec{V}_{\wp j}^{(v o r t)} \Gamma
$$

where $\vec{V}_{\wp j}$ and $\vec{V}_{\wp j}^{(v o r t)}$ are the influence coefficient vectors of panel $j$ on point $\wp \circ$.

An example of the panel geometry used for the centre body and cowl flow analysis is shown in figure 3.2 and represents the air intake geometry at symmetry plane 1 (Fig 2.1). Because an axisymmetric panel method is used for the centre body and cowl surface flow analysis at symmetry plane 1 , the centre body and cowl surfaces are assumed to be axisymmetric bodies. Therefore, in the discussion of the flow analysis around the centre body and cowl surfaces, the height of points along each surface above the intake centre line is expressed in radius, $r$, rather than $y$ coordinate as is used in Section 2.

An example of the panel geometry for the fairing flow analysis is shown in figure 3.3 
and represents a flattened constant radius slice of the fairing geometry at the plane 2 area average radius and is bounded by symmetry plane 1 on both sides. Since the fairing panel geometry is a flattened constant radius slice of the fairing geometry, a 2D panel method is used for the fairing flow analysis.

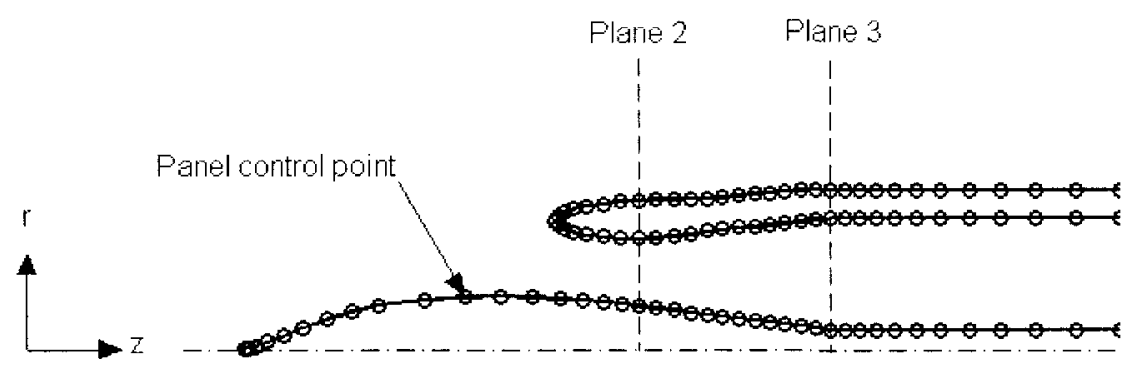

Figure 3.2: Example axisymmetric panel geometry along symmetry plane 1
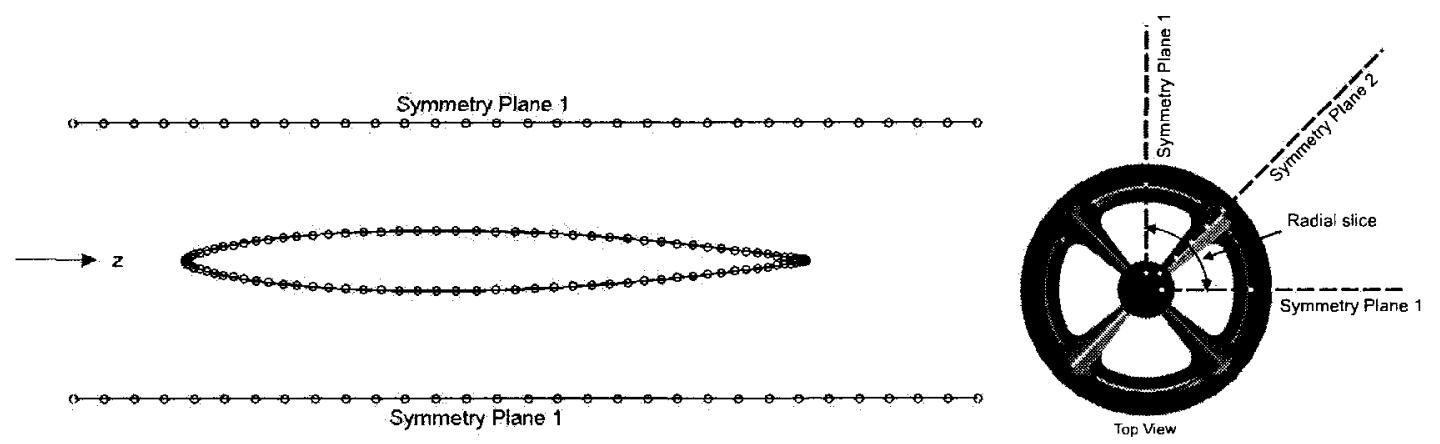

Figure 3.3: Example 2D fairing panel geometry

The number of panels used for both panel methods is set by first specifying the number of panels used along the centre body in region $\mathrm{C}$ (Fig 2.5), $N_{C B C}$. The panel density in region $\mathrm{C}$ is then used to determine the number of panels used on the remaining portions of the intake geometry. The panel distribution over each intake region is specified using the one-sided and two-sided exponential bunching laws described in the ICEM 11.0 documentation [41]. 
With the exception of the calculation of the velocity influence coefficients, the calculation procedure for the axisymmetric and 2D panel methods is identical. The velocity influence coefficients differ between the two panel methods because the axisymmetric method uses ring shaped potential flow elements whereas the 2D method uses line potential flow elements. In the 2D panel method, it is convenient to express the velocity influence coefficient equations in terms of a local panel element coordinate system, whereas in the axisymmetric panel method, the velocity influence coefficients are simply expressed in the original z-r coordinate system. Detailed information on the 2D panel method is provided by Hess [23]. The following section will describe the axisymmetric panel method in greater detail.

\section{Axisymmetric Panel Method}

The axisymmetric panel method used in this work represents surface panels as parabolic arcs with linearly varying source strengths. An example of how the intake surfaces are represented using parabolic shaped panels is illustrated in figure 3.4. Each surface panel is bounded by two end points which lie exactly on the surface the panels are representing, and a control point at the mid point of each surface panel. At the control point of each panel, the zero normal velocity boundary condition is enforced. The location of the surface panel

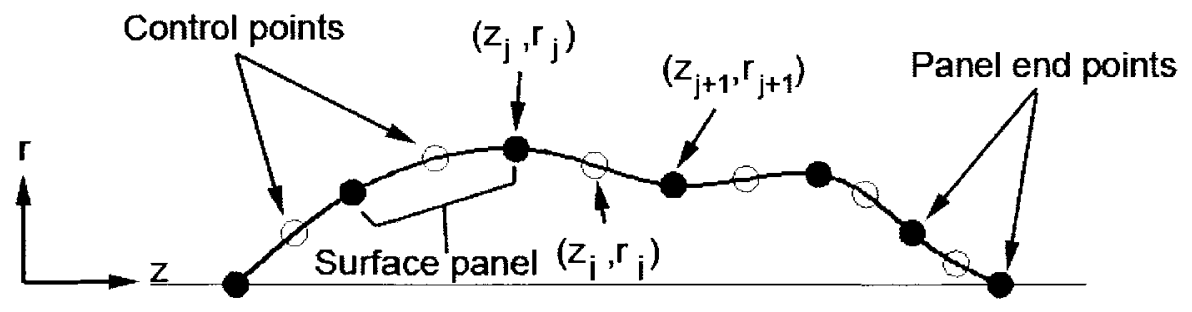

Figure 3.4: Body contour surface panels with control points

control point is defined as the point where the normal vector extending from the mid point of a flat panel between points $\left(z_{j}, r_{j}\right)$ and $\left(z_{j+1}, r_{j+1}\right)$, and the surface panel arc, intersect, 
shown in figure 3.5. The slope of the tangent line at the control point is then defined as the

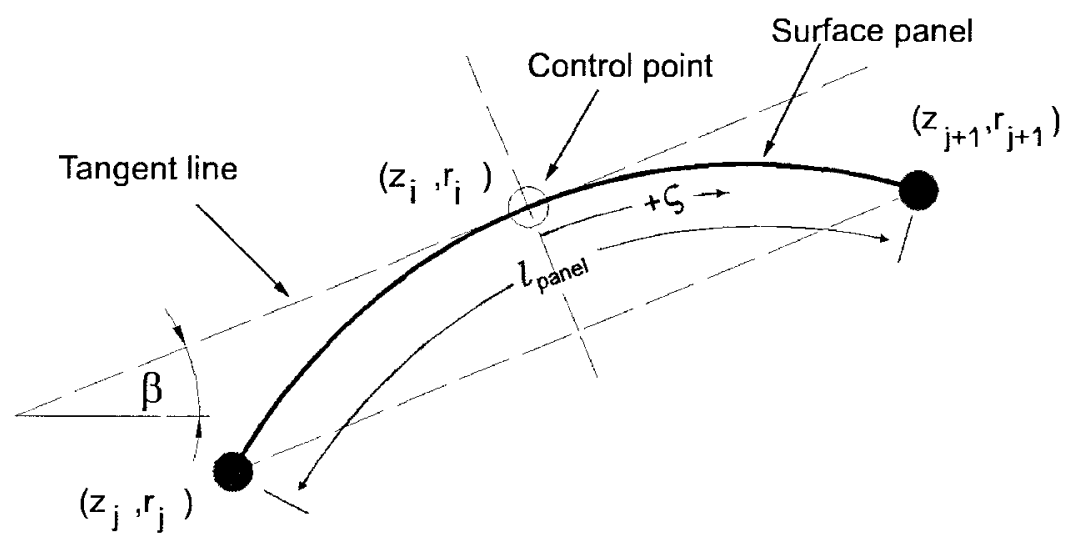

Figure 3.5: Surface panel geometry

slope, $\beta$, of the surface panel.

Once the panel geometry is defined, the ring-source and ring-vortex velocity influence coefficient vectors, $\vec{V}_{i j}$ and $\vec{V}_{i j}^{(v o r t)}$, which are a function of panel geometry, can be calculated. The linear ring-source strength distribution on panel $j$ at panel surface location $\varsigma$ is defined as

$$
\lambda_{j}(\varsigma)=\lambda_{j}+\lambda^{\prime} \varsigma
$$

where $\lambda_{j}$ and $\lambda_{j}^{\prime}$ represent the ring-source strength and first derivative of the ring-source strength distribution respectively at the control point of panel $j$, and $\zeta$ is the surface distance between a point along the surface panel and the panel control point and is positive in the direction shown in figure 3.5. The disturbance velocity at panel $i$ due to the ring-source and ring-vortex on panel $j$ is then

$$
\vec{u}_{i j}=\vec{V}_{i j} \lambda_{j}+\vec{V}_{i j}^{(\text {vort })} \Gamma=\vec{\forall}_{i j} \lambda_{j}+\vec{\forall}_{i j}^{\prime} \lambda_{j}^{\prime}+\vec{V}_{i j}^{(\text {vort })} \Gamma
$$

where $\vec{\forall}_{i j}$ and $\vec{\forall}_{i j}^{\prime}$ are the intermediate ring-source velocity influence coefficient vector 
and its first derivative respectively, and $\vec{V}_{i j}^{(v o r t)}$ is the ring-vortex influence coefficient vector. The ring-source velocity influence coefficient vector $\vec{V}_{i j}$, is a combination of $\vec{\forall}_{i j}$ and $\vec{\forall}_{i j}^{\prime}$. Because there cannot be more than one unknown source strength term associated with each surface panel to properly solve the source and vortex strengths, the affect of the term $\vec{\forall}_{i j}^{\prime} \lambda_{j}^{\prime}$ is calculated based on the geometry of panel $j$ as well as the adjacent panels $(j-1)$ and $(j+1)$. Details of the calculation of $\vec{\forall}_{i j}^{\prime} \lambda_{j}^{\prime}$ can be found in the work by Hess [23,25].

It is convenient to break down $\vec{\forall}_{i j}$ into its $z$ and $r$ components, $\forall_{z_{i j}}$ and $\forall_{r_{i j}}$, where

$$
\begin{aligned}
& \forall_{z_{i j}}=\vec{\forall}_{i j} \cdot \hat{z} \\
& \forall_{r_{i j}}=\vec{\forall}_{i j} \cdot \hat{r}
\end{aligned}
$$

In order to determine the $z$ and $r$ influence coefficients, $\forall_{z_{i j}}$ and $\forall_{r_{i j}}$, of an entire surface panel on a given location, the influence of the ring-source distribution at several discrete points along the surface panel must first be calculated. The number of sub-segments, $N_{i j \text { seg }}$, used for this numerical integration is set as $16\left(l_{\text {panel }} / r_{\text {min }}\right)$ rounded to the nearest even integer up to a maximum of 50 sub-segments, where $r_{\min }$ is the distance between the $i$-th control point and the nearest endpoint of the $j$-th panel. This relation has been determined through trial and error by Hess [21] to produce good results. Figure 3.6 shows

$$
N_{\mathrm{ij}_{\mathrm{seg}}}=6
$$

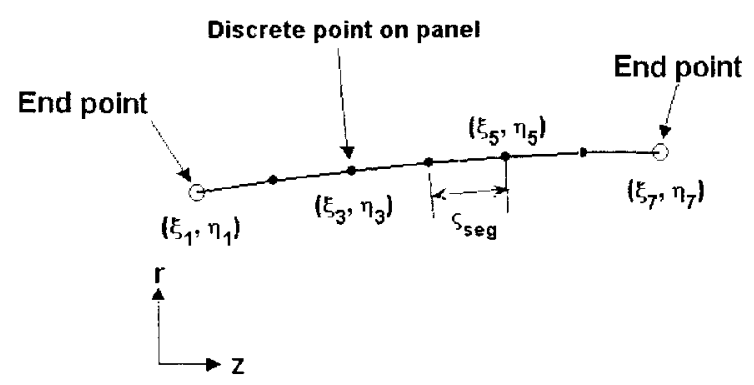

Figure 3.6: Breakdown of panel $j$ into $N_{i j_{s e g}}$ sub-segments. $N_{i j_{\text {seg }}}=6$.

a surface panel broken down into sub-segments. The local influence coefficients for the 
discrete points on the given panel are then numerically integrated over the entire panel to give the intermediate ring-source velocity influence coefficients, $\forall_{z_{i j}}$ and $\forall_{r_{i j}}$. The local velocity influence coefficients, $V_{z_{\left.i j_{\xi}, \eta\right)}^{\oplus}}^{\oplus}$ and $V_{r_{i j}(\xi, \eta)}^{\oplus}$, of point $(\xi, \eta)$ on surface panel $j$, on control point $i$ are calculated as described by Hess [21]

$$
\begin{gathered}
V_{z_{i_{j}(\xi, \eta)}}^{\oplus}=\frac{4 \eta\left(z_{i}-\xi\right) E(k)}{\left[\left(r_{i}-\eta\right)^{2}+\left(z_{i}-\xi\right)^{2}\right] \sqrt{\left(r_{i}+\eta\right)^{2}+\left(z_{i}-\xi\right)^{2}}} \\
V_{r_{i j}(\xi, \eta)}^{\oplus}=\frac{2 \eta}{r_{i} \sqrt{\left(r_{i}+\eta\right)^{2}+\left(z_{i}-\xi\right)^{2}}\left(K(k)+\frac{r_{i}^{2}-\eta^{2}-\left(z_{i}-\xi\right)^{2}}{\left(r_{i}-\eta\right)^{2}+\left(z_{i}-\xi\right)^{2}} E(k)\right)} \\
k=\sqrt{\frac{4 \eta r_{i}}{\left(r_{i}+\eta\right)^{2}+\left(z_{i}-\xi\right)^{2}}}
\end{gathered}
$$

where $\xi$ and $\eta$ are the $z$ and $r$ coordinates of the discrete points along surface panel $j, z_{i}$ and $r_{i}$ are the coordinates of the control point $i$, and $K(k)$ and $E(k)$ are the complete elliptic integrals of the first and second kind respectively. The complete elliptic integral equations are shown below

$$
\begin{aligned}
& K(k)=\int_{0}^{\pi / 2} \frac{d \psi}{\sqrt{1-k^{2} \sin ^{2} \psi}} \\
& E(k)=\int_{0}^{1} \sqrt{1-k^{2} \sin ^{2} \psi} d \psi
\end{aligned}
$$

where $d \psi$ is the variable of integration. In this work, solution of the complete elliptic integrals is accomplished through a MATLAB ${ }^{\circledR}$ 7.0.1 function.

The intermediate ring-source influence coefficient components of the entire $j$-th panel at the $i$-th control point, are then calculated by numerically integrating the results from Eqns 3.11, 3.12 over the entire $j$-th surface panel using Simpson's rule, as shown for $\forall_{z i j}$ 


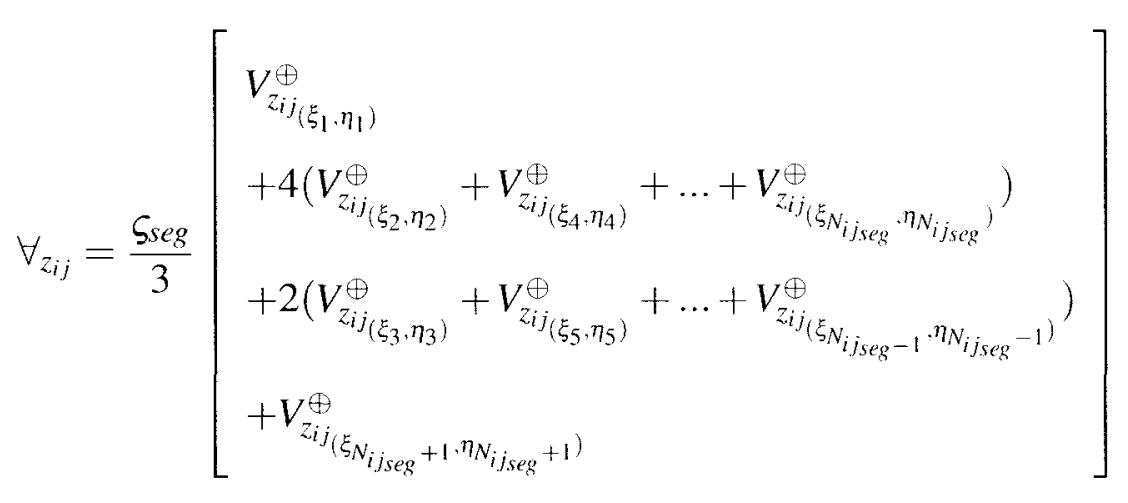

The local, constant-strength ring-vortex influence coefficients, $V_{z_{i j}(\xi, \eta)}^{\oplus(v o r t)}$ and $V_{r_{i j}(\xi, \eta)}^{\oplus(v o r t)}$ are related to the local ring-source influence coefficients by [25]

$$
\begin{gathered}
V_{z_{i j_{\xi}, \eta}}^{\oplus(\text { vort })}=\frac{\eta^{2}-r_{i}^{2}}{\eta\left(z_{i}-\xi\right)} V_{z_{i j_{\xi}, \eta}}^{\oplus}+\frac{r_{i}}{\eta} V_{r_{i j_{\xi}, \eta}}^{\oplus} \\
V_{r_{i j_{\xi}, \eta}}^{\oplus(\text { vort })}=\frac{r_{i}}{\eta} V_{z_{i j_{\xi}, \eta}}^{\oplus}-\frac{z_{i}-\xi}{\eta} V_{r_{i j_{\xi}, \eta}}^{\oplus}
\end{gathered}
$$

The vortex influence coefficients $V_{z_{i j}}^{(v o r t)}$ and $V_{r_{i j}}^{(v o r t)}$ are then solved for in the same manner as the source influence coefficients using the Simpson's rule to numerically integrate over the surface panels.

A special case arises when considering a panels influence on its own control point (i.e. $i=j$ ). In this situation, where $z_{i}=\xi$ and $r_{i}=\eta$, Eqns $3.11,3.12,3.13$ become singular and can no longer be integrated. In these cases, the influence of a panel on its own control point is calculated by dividing the panel into three sub-elements as shown in figure 3.7. There are three contributions to the total influence of a panel on its own control point. The first of these contributions come from the outer sub-elements, and the second contribution comes from the centre sub-element. In the outer sub-elements, the local influence coefficients are calculated using Eqns 3.11-3.13 since $z_{i} \neq \xi$ and $r_{i} \neq \eta$. The outer sub-element influence coefficients, $V_{z_{i i}}^{(\text {outer })}$ and $V_{r_{i i}}^{(\text {outer })}$, are then calculated by numerically integrating 


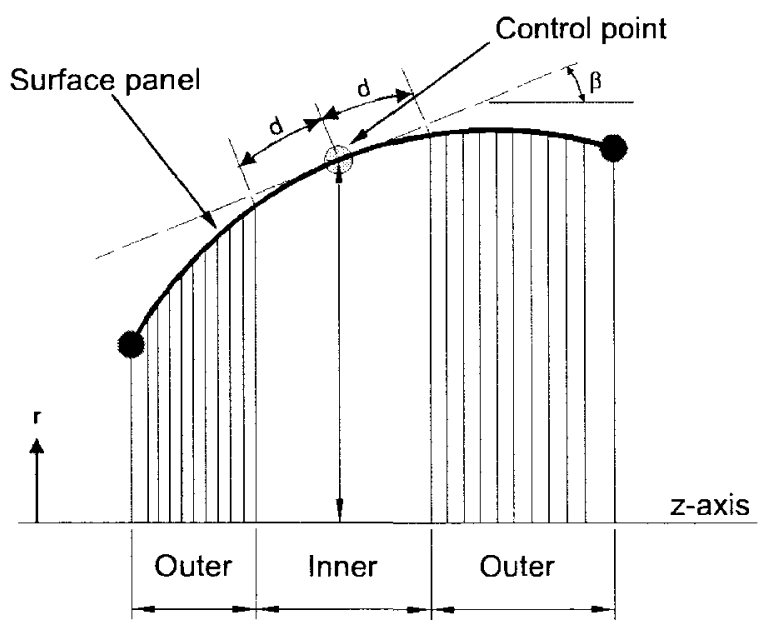

Figure 3.7: Sub-element breakdown of $i$-th panel

Eqns 3.11- 3.13 over these sub-elements. For the centre sub-element, Eqns 3.11- 3.13 must be expanded in a power series to remove the singularity condition. These power series are then integrated between $\varsigma=-d$ and $\varsigma=d$ to give the influence coefficients, $V_{z i i}^{\odot}$ and $V_{r_{i i}}^{\odot}$, of the singular sub-element on the panel's control point. The equations for these power series equations are provided by Hess [25]. The length $d$ has been determined through trial and error [21] and is given below

$$
d= \begin{cases}0.08 r_{i} & \text { if } 0.08 r_{i}<l_{\text {panel }} / 2 \\ l_{\text {panel }} / 2 & \text { if } 0.08 r_{i}>l_{\text {panel }} / 2\end{cases}
$$

where $r_{i}$ is the radius of panel $i$ 's control point above the centre line. The final contributions to the influence of a panel on its own control point are $V_{z i i}^{\otimes}$ and $V_{r_{i i}}^{\otimes}$ and are defined by Hess [25] as

$$
\begin{aligned}
& V_{z_{i i}}^{\otimes}=-2 \pi \sin \beta \\
& V_{r_{i i}}^{\otimes}=2 \pi \cos \beta
\end{aligned}
$$

Therefore, the intermediate influence coefficients of a panel on its own control point are 
the sum of these three contributions so that

$$
\begin{aligned}
& \forall_{z_{i i}}=V_{z_{i i}}^{(\text {outer })}+V_{z_{i i}}^{\odot}+V_{z_{i i}}^{\otimes} \\
& \forall_{r_{i i}}=V_{r_{i i}}^{(\text {outer })}+V_{r_{i i}}^{\odot}+V_{r_{i i}}^{\otimes}
\end{aligned}
$$

\subsubsection{Combination Procedure}

Simulation of the flow through and around an air intake using a potential flow panel method is complicated by the need to enforce the required freestream flight conditions while also enforcing the desired mass flow rate through the intake. This mass flow rate is primarily determined by the flow conditions created by the device the intake is attached to. In the case of an RBCC ejector section operating at subsonic freestream velocities, the intake air mass flow rate is dependant on the rocket exhaust velocity, pressure, and the mixing between the air and rocket exhaust streams. In this work, the required freestream flow conditions are determined from the flight condition input parameters $M_{\infty}$ and $h_{A l t}$, which are determined based on the vehicles position along the vehicle flight profile (Fig 3.1). The desired mass flow rate in this work is simply the mass flow rate through the intake assuming inviscid flow and a static pressure at plane 3 of $p_{3}$, which is a flight condition input parameter.

The plane at which this desired mass flow rate must be achieved is called the control plane, identified in figure 3.8 , and corresponds to air intake plane 3 . In potential flow methods, the desired mass flow rate is expressed in terms of the average control plane velocity in the $z$ direction, $\bar{U}_{z_{3}}$, and the control plane area and the average local density. The value of $\bar{U}_{z_{3}}$ is calculated by taking the average of the $z$ component of the velocity at 30 evenly distributed points along the control plane using Eqn. 3.7 as well as the surface velocities along the intake surfaces at the control plane using Eqn. 3.6 and is calculated as 
follows

$$
\bar{U}_{z_{3}}=\left(\sum_{l=1}^{30} U_{z_{3 z_{l}}}+U_{C B_{z_{3}}}+U_{C W_{z_{3}}}\right) / 32
$$

where $U_{\left.z_{\xi}\right\}}$ is the $z$ component of the velocity at point $\wp$ along the control plane and $U_{C B_{33}}$ and $U_{C W_{z 3}}$ are the $z$ components of the surface velocity along the centre body and cowl surfaces respectively.

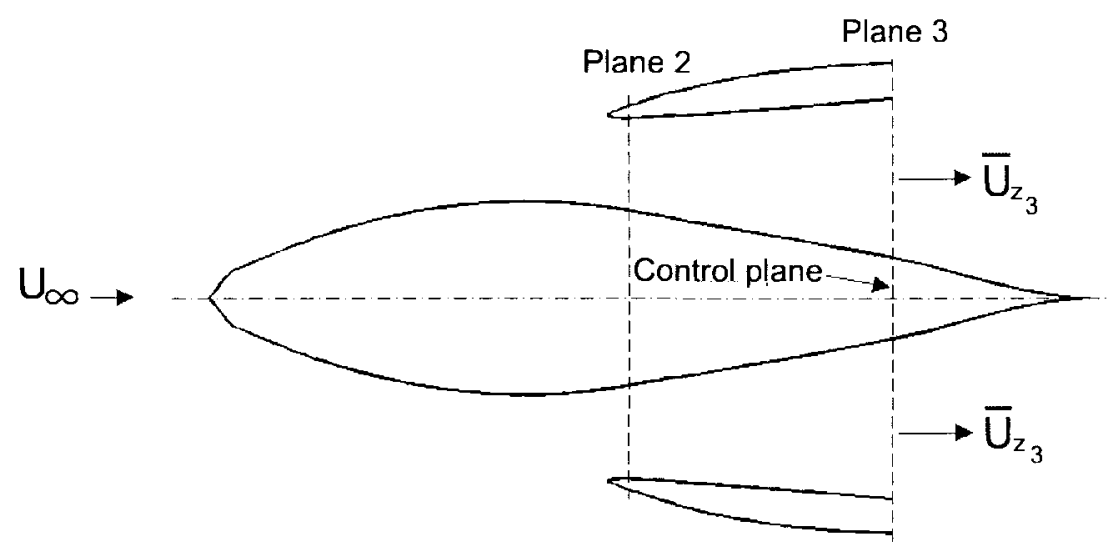

Figure 3.8: Air intake cross-section view

A potential method for enforcing the desired mass flow rate at the control plane is to place panels along the control plane and set the normal velocity on these panels to $\bar{U}_{z_{3}}$ instead of the zero normal velocity condition. However, this method leads to unrealistic flow velocities near the control plane due to the panel method's inability to properly handle sharp internal corners [25] as well as incorrect surface velocities along the exterior surface of the cowl. Instead, the surface vorticity is used along the intake surfaces to draw the flow into the intake as shown in figure 3.9. The opposing direction of the surface vorticity along the centre body and cowl surfaces in figure 3.9 is achieved by specifying the sign of the vortex influence coefficients due to panels along the centre body such that they are opposite sign to the cowl panels.

In order to correctly enforce the desired mass flow rate as well as the desired freestream velocity far upstream of the air intake, a combination procedure described by Hess [25] 
is used which uses surface vorticity and a modified panel geometry. The panel geometry is modified such that the bodies downstream of the control plane form constant diameter afterbodies as shown in figure 3.9. This geometry modification allows one to avoid the difficulties with internal corners. While this geometry modification neglects the centre body in region $\mathrm{D}$, this type of geometry modification has been applied to axisymmetric ducts with center bodies by Hess [21] and surface velocity results match well with experiments.

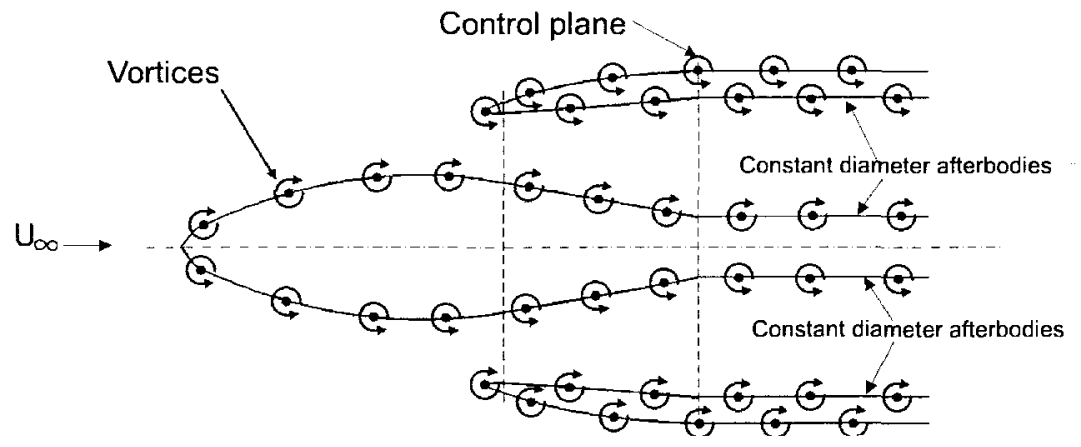

Figure 3.9: Modified air intake geometry for panel method with constant diameter after body sections

With this modified panel geometry, simulating the desired mass flow rate and freestream velocity around the intake is achieved through a combination of two flow situations which are illustrated in figure 3.10 .

The first flow situation is the air intake operating with a unit onset flow at $0^{0}$. The second flow condition is a static flow situation where vortex strength along the surface panels is given a value of unity, which simulates a flow where the freestream velocity at infinity is zero but there is a non-zero mass flow rate at the control plane. Because the final flow solution is calculated by scaling and combining these two flow situations, the value of the freestream velocity used in flow situation 1 and the vortex strength in flow situation 2 can be set arbitrarily and are set to unity for convenience.

The solutions to both flow situations are scaled and combined such that the desired adjusted incompressible freestream velocity at infinity, $\vec{U}_{\infty}^{\diamond}$ and the adjusted incompressible 


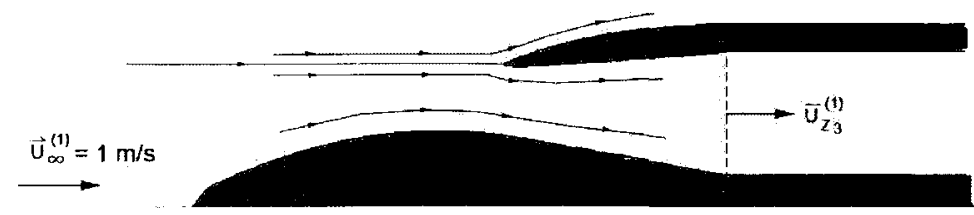

Flow Situation 1

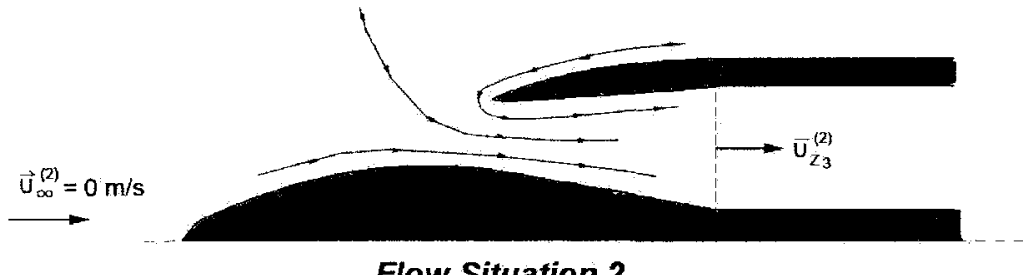

Flow Situation 2

Figure 3.10: Visual representation of two flow situations used in combination procedure.

control plane velocity, $\bar{U}_{z_{3}}^{\diamond}$, are obtained. The adjusted incompressible velocities are equal to the desired compressible flow velocity multiplied by the local static to total density ratios and are calculated below

$$
\begin{aligned}
& \vec{U}_{\infty}^{\diamond}=\vec{U}_{\infty}^{(c o m p)} \frac{\rho_{\infty}^{(c o m p)}}{\rho_{0}} \\
& \bar{U}_{z_{3}}^{\diamond}=\bar{U}_{z_{3}}^{(c o m p)} \frac{\rho_{z}^{(c o m p)}}{\rho_{0}}
\end{aligned}
$$

where $\vec{U}_{\infty}^{(c o m p)}, \bar{U}_{z_{3}}^{(c o m p)}, \rho_{\infty}^{(c o m p)}$, and $\rho_{z_{3}}^{(c o m p)}$ are the compressible flow properties and are known from the flight condition input parameters.

The adjusted incompressible flow velocities are used as boundary conditions so that when compressibility corrections are applied, the freestream and control plane velocities match the actual compressible target values, $\vec{U}_{\infty}^{(c o m p)}$ and $\bar{U}_{z_{3}}^{(c o m p)}$.

The solution procedure for each of the two flow situations differs slightly. In order to solve flow situation 1 with a uniform onset flow, the $(N) \times(N+1)$ system of equations from Eqn 3.5 must be solved. Recalling that the velocity influence coefficient matrix is an $(N) \times(N+1)$ matrix, the required $(N+1)^{t h}$ row in the coefficient matrix is calculated 
by enforcing the Kutta condition at the intake lip leading edge. The Kutta condition is enforced by forcing the velocity magnitude on either side of the lip leading edge, shown in figure 3.11 to be equal. The Kutta condition is enforced using the following equation

$$
\begin{aligned}
& \left(\sum_{j=1}^{N} \vec{V}_{\|_{\left(i_{l i p}, j\right)}}+\sum_{j=1}^{N} \vec{V}_{\|_{\left(\left(i l_{l i p}+1\right) . j\right)}}\right) \lambda_{j} \\
& +\left(\sum_{j=1}^{N} \vec{V}_{\|_{\left(i_{l i p}, j\right)}^{(v o r t)}}+\sum_{j=1}^{N} \vec{V}_{\|_{\left(\left(i_{l i p},+1\right) . j\right)}^{(v o r t)}}\right) \Gamma \\
& =-\left(U_{\infty \|_{\left(i_{l i p}\right)}}+U_{\infty \|_{\left(i_{l i p}+1\right)}}\right)
\end{aligned}
$$

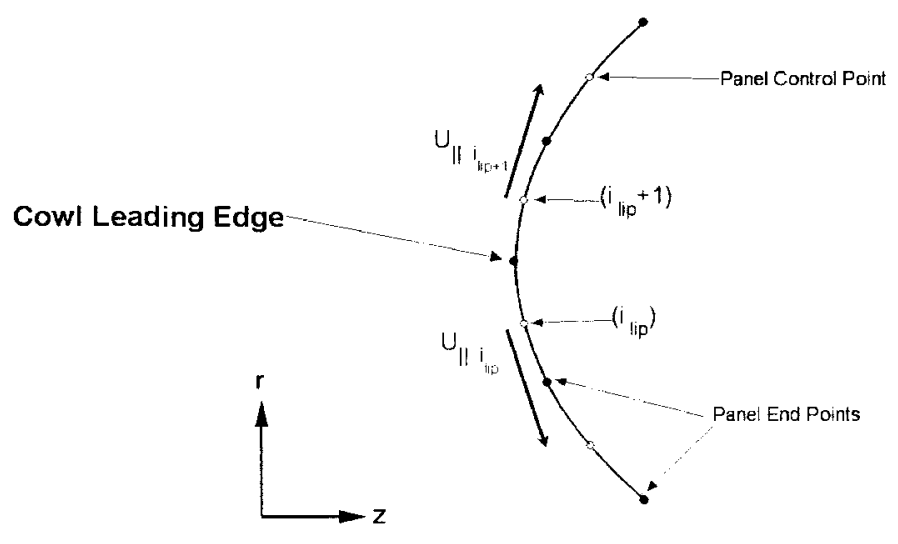

Figure 3.11: Zoomed in view of cowl leading edge panel geometry.

where the indices $i_{l i p}$ and $\left(i_{l i p}+1\right)$ are the indices of the surface panels that are located below and above the lip leading edge point, $\vec{V}_{\left.\|_{(i l i p}, j\right)}$ and $\vec{V}_{\|_{\left.\left(i i_{l i p},+1\right), j\right)}}$ are the tangential velocity influence coefficients of panel $j$ acting on the lip surface panels, and $U_{\infty} \|_{(i \text { lip })}$ and $U_{\infty} \|_{\left(i_{l i p}+1\right)}$ are the tangential velocity components at the lip surface panels due to the freestream velocity vector.

With the Kutta condition enforced, Eqn. 3.23 can be used with Eqn 3.5 to form a $(N+1) \times(N+1)$ system of equations. A Gaussian elimination procedure is then used to solve for the $N$ source strengths and the 1 vortex strength. Because the final flow field, with the correct $\vec{U}_{\infty}^{(c o m p)}$ and $\bar{U}_{-3}^{(c o m p)}$, is obtained through a combination of two flow solutions, 
the actual value of $\vec{U}_{\infty}$ used to solve the first flow condition is not critical and is arbitrarily set to a value of 1 .

Flow situation 2, the static situation, is solved using a modified version of Eqn 3.5. In the static flow situation, $\vec{U}_{\infty}$ is equal to zero. In addition, because the solution to this flow condition will be used in the combination procedure, the vortex strength $\Gamma$ is set to a value of 1 . Noting the above conditions, Eqn 3.5 can be modified to

$$
\sum_{j=1}^{N} \vec{V}_{\perp_{i j}} \lambda_{j}=-\sum_{j=1}^{N} \vec{V}_{\perp_{i j}}^{(v o r t)}
$$

Because $\Gamma$ has been removed as an unknown, Eqn 3.24 is an $(N \times N)$ system of linear equations and the source strengths can be solved without any additional boundary conditions. The surface velocities at each surface panel for each flow situation can then be solved using Eqn 3.6.

The solutions to the two flow situations are then scaled using the combination coefficients $\varphi_{(1)}$ and $\varphi_{(2)}$, and then combined such that the correct $\vec{U}_{\infty}^{\diamond}$ and $\bar{U}_{8,3}^{\diamond}$ values are obtained. The combined velocity vector at surface panel $i$ can be expressed as

$$
\vec{U}_{i}^{(c o m b)}=\varphi_{(1)} \vec{U}_{i}^{(1)}+\varphi_{(2)} \vec{U}_{i}^{(2)}
$$

where $\varphi_{(1)}$ and $\varphi_{(2)}$ are the combination coefficients for flow situations (1) and (2) respectively, $\vec{U}^{(1)}$ and $\vec{U}^{(2)}$ are the incompressible velocity vectors from the two flow situations, and $\vec{U}_{i}^{(c o m b)}$ is the final adujusted incompressible flow velocity. The combination coefficients are solved for using the system of equations below

$$
\begin{aligned}
& \varphi_{(1)} \vec{U}_{\infty}^{(1)}+\varphi_{(2)} \vec{U}_{\infty}^{(2)}=\vec{U}_{\infty}^{\diamond} \\
& \varphi_{(1)} \bar{U}_{z 3}^{(1)}+\varphi_{(2)} \bar{U}_{z 3}^{(2)}=\bar{U}_{z_{3}}^{\diamond}
\end{aligned}
$$


where $\bar{U}_{z_{3}}^{(1)}$ and $\bar{U}_{z_{3}}^{(2)}$ are the average flow velocities at the control plane for flow situations (1) and (2).

\subsubsection{Velocity Scaling}

Although the centre body and cowl surfaces have been defined so that the specified $A R$ is met for the 3D intake geometry, the surface velocities calculated using the axisymmetric panel method and the combination procedure assume the intake geometry is axisymmetric. However, due to the presence of the fairing geometry, which reduces the flow area through the duct when compared to an axisymmetric geometry, corrections must be made to the calculated surface velocities.

The area discrepancy between the axisymmetric panel geometry and the true intake geometry means that the panel method will calculate the flow assuming a larger $A R$ $\left(A_{a x i_{2}}>A_{2}\right)$ than is present for the actual geometry. This causes the axisymmetric panel method to calculate a lower plane 2 velocity than will be seen for the actual 3D geometry. Therefore, the velocity scaling method used by McFarland [42] can be used to account for this difference as follows. Since $\dot{m}$ is constant through the duct, and the calculated surface velocities are incompressible, the actual surface velocity at a given axial location can be estimated based on the ratio between the axisymmetric and real flow areas. Since the velocity at plane 3 is fixed and is a boundary condition for the panel method, the scaling factor is referenced to the conditions at this plane. The scaled incompressible velocity, $\vec{U}_{i}^{(s c a l e d)}$, at panel control point $i$ is calculated as

$$
\vec{U}_{i}^{(\text {scaled })}=\frac{\frac{A_{a x i_{i}}}{A_{a x i_{3}}}}{\frac{A_{i}}{A_{3}}} \vec{U}_{i}^{(\text {comb })}
$$

where $A_{a x i_{i}}$ is the flow area at control point $i$ assuming no fairing geometry, and $A_{i}$ is the actual flow area at control point $i$. In centre body region A and region B (Fig 2.5), where 
the fairing is present but the flow is not completely enclosed, the area ratio used for velocity scaling is determined by specifying the upper radial bound of the intake streamtube, illustrated in figure 3.12, and then applying Eqn. 3.27 to this streamtube. The radius of the streamtube at the downstream boundary is simply set to be coincident to the radius of the cowl leading edge. The radius of the streamtube at the upstream boundary, $r_{s t_{\infty}}$, at the centre body nose, is calculated based on the predicted flow capture ratio, which is defined as

$$
F C=\frac{A_{\infty}}{A_{C W_{L E}}}=\frac{U_{C W_{L E}} \rho_{C W_{L E}}}{U_{\infty} \rho_{\infty}}
$$

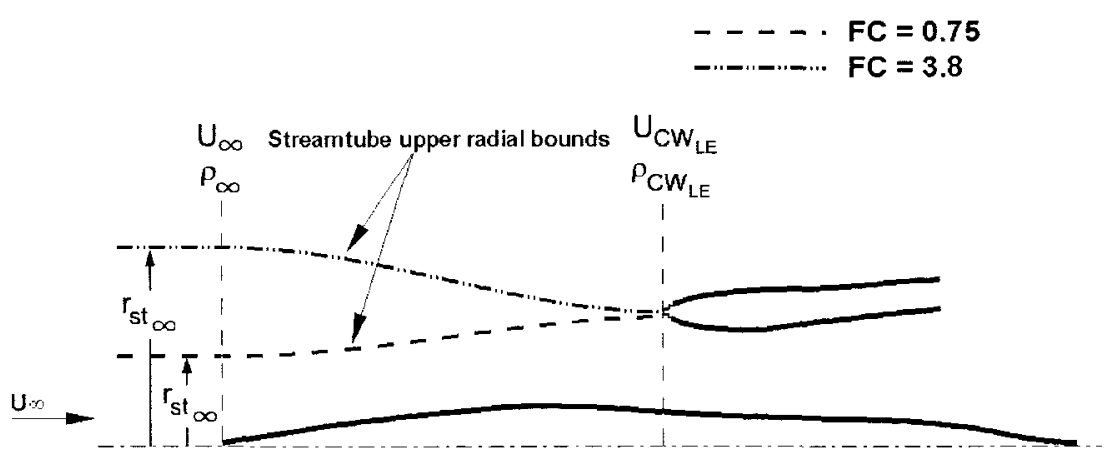

Figure 3.12: Streamtube bounds for different $F C$

where $\rho_{\infty}$ and $\rho_{C W_{L E}}$ are the local densities at the upstream and downstream streamtube boundaries respectively. While compressibility effects have not been addressed in the flow analysis up to this point, compressibility is used in the $F C$ ratio calculation to improve the accuracy of the calculation of $r_{s t_{\infty}}$. The density at infinity, $\rho_{\infty}$ can be determined from the freestream flight conditions. The density at the cowl leading edge plane can be determined from the known $\bar{U}_{z 3}^{(\text {comp })}$, and thus Mach number at plane 3, the known flow areas $A_{3}$ and $A_{C W_{L E}}$, and the isentropic Mach number relations. The radius of the streamtube at the 
furthest point upstream is then

$$
r_{s t_{\infty}}=\left(\frac{A_{\infty}}{\pi}\right)^{\frac{1}{2}}
$$

The complete upper radial bound of the external streamtube is then defined using a hermite curve with boundary slopes equal to zero, as shown in figure 3.12 .

\subsubsection{Compressibility Correction}

The Lieblein-Stockman [27] compressibility correction is applied to the final scaled incompressible velocities to account for compressibility affects. It has been developed for internal flows, but has also been adapted to external flow situations by Dietrich et al [28]. Flow around the air intake geometry includes regions of external and internal flow. The compressible velocity, $U_{i}^{(c o m p)}$, is calculated from the equations

$$
U_{i}^{(\text {comp })}=U_{i}^{(\text {scaled })}\left(\frac{\rho_{0}}{\bar{\rho}_{i}^{(c o m p)}}\right)^{m}
$$

where $m$ is

$$
m=\frac{U_{i}^{(\text {scaled })}}{\bar{U}_{i}^{(\text {scaled })}}
$$

where $U_{i}^{(\text {scaled })}$ is the local surface velocity.

Because $\bar{\rho}_{i}^{(c o m p)}$ is unknown, a relation between the incompressible velocity $\bar{U}_{i}^{(\text {scaled })}$ and $\bar{\rho}_{i}^{(c o m p)}$ must be found. Using continuity,

$$
\frac{\bar{U}_{i}^{(\text {scaled })}}{U^{(\text {comp }) *}}=\frac{\bar{\rho}_{i}^{(\text {comp })}}{\rho_{0}}\left(\frac{\bar{U}_{i}^{(\text {comp })}}{U^{(c o m p) *}}\right)
$$


where the superscript $*$ indicates a critical (sonic) flow property. Next, using the isentropic expressions for the critical velocity ratio and the local to total density ratio, the ratio $\frac{\bar{U}_{i}^{(c o m p)}}{U^{(c o m p) *}}$ in Eqn. 3.32 can be expressed as

$$
\frac{\bar{U}_{i}^{(c o m p)}}{U^{(c o m p) *}}=\left(\frac{(\gamma+1)}{(\gamma-1)}\left[1-\left(\frac{\bar{\rho}_{i}^{(c o m p)}}{\rho_{0}}\right)^{(\gamma-1)}\right]\right)^{\frac{1}{2}}
$$

Combining Eqn. 3.32 and Eqn. 3.33, and noting that $U^{(\mathrm{comp}) *}=a^{*}$, the following expression is obtained

$$
\frac{\bar{U}_{i}^{(c o m p)}}{U^{(c o m p) *}}=\frac{\bar{U}_{i}^{(c o m p)}}{a^{*}}=\frac{\bar{\rho}_{i}^{(c o m p)}}{\rho_{0}}\left[\left(\frac{(\gamma+1)}{(\gamma-1)}\left[1-\left(\frac{\bar{\rho}_{i}^{(c o m p)}}{\rho_{0}}\right)^{(\gamma-1)}\right]\right)^{\frac{1}{2}}\right]
$$

Rearranging Eqn. 3.34, and noting that for air, $a^{*}=0.9219 a_{0}$, an expression for the density ratio can be found as

$$
\frac{\bar{\rho}_{i}^{(\text {comp })}}{\rho_{0}}=\left(1-\frac{\gamma-1}{\gamma+1}\left(\frac{\bar{U}_{i}^{(\text {scaled })}}{0.9219 a_{0}}\right)^{2}\left(\frac{\rho_{0}}{\bar{\rho}_{i}^{(c o m p)}}\right)^{2}\right)^{\frac{1}{\gamma-1}}
$$

where $\bar{U}_{i}^{(\text {scaled })}$ is the scaled mean velocity along a plane at control point $i$, and $a_{0}$ is the total sonic speed and is found from the known freestream flow conditions. The value of $\bar{\rho}_{i}^{(c o m p)}$ can be found using Eqn. 3.35.

The calculation of $\bar{U}_{i}^{(\text {scaled })}$ differs depending on if one is modeling internal or external flow. In the case of internal flows such as in the duct portion of the air intake, $\bar{U}_{i}^{(s c a l e d)}$ is simply the mean scaled velocity across a plane at the axial location of control point $i$ and is calculated in the same manner as in Eqn. 3.21. For external flows Dietrich et al [28] have 
found that $\bar{U}_{i}^{(\text {scaled })}$ can be calculated as a weighted average between $U_{\infty}^{(c o m p)}$ and $U_{i}^{(s c a l e d)}$ and is calculated as follows

$$
\bar{U}_{i}^{(\text {scaled })}=\frac{\rho_{\infty}^{(c o m p)}}{\rho_{0}} U_{\infty}^{(\text {comp })}+\left(1-\frac{\rho_{\infty}^{(\text {comp })}}{\rho_{0}}\right) U_{i}^{(\text {scaled })}
$$

\subsection{Viscous Flow Solver}

The viscous flow solver step takes the inviscid, compressible surface velocities calculated from the inviscid flow solution and uses them as a boundary condition to solve the boundary layer development along the intake surfaces as well as the total pressure loss due to friction. The following sections will describe the boundary layer method used and the total pressure loss calculations.

\subsubsection{Integral Boundary Layer Method}

Childs' [20] compressible turbulent integral boundary layer method is used in this work. This method is derived from Head's entrainment method [34] and uses the momentum integral equation and the entrainment equation. The momentum equation for compressible flow along an axisymmetric body is given below as

$$
\frac{d \theta}{d s}+\left(2+H-M^{2}\right) \frac{\theta}{U^{(c o m p)}} \frac{d U^{(c o m p)}}{d s}=\frac{C_{f}}{2}-\frac{\theta}{r} \frac{d r}{d s}
$$

where $\theta$ is momentum thickness, $s$ is the distance along the surface starting from the turbulent boundary layer start point, $H=\frac{\delta^{*}}{\theta}$ is the conventional boundary layer shape factor, $M$ is the flow Mach number at the upper boundary of the boundary layer, $U^{(c o m p)}$ is the compressible flow velocity at the upper boundary of the boundary layer, $C_{f}$ is the local skin friction coefficient, and $r$ is the local radius of the axisymmetric body surface. The 
flow velocity $U^{(c o m p)}$ and thus Mach number $M$ are known from the inviscid flow solution. The entrainment equation is given below as

$$
\left(\frac{1}{\rho U^{(c o m p)} r}\right) \frac{d}{d s}\left(\rho U^{(c o m p)} r\left(\delta-\delta^{*}\right)\right)=E
$$

where $\delta$ is the boundary layer thickness, $\delta^{*}$ is the displacement thickness, $r$ is the radius of the surface the boundary layer is forming on, and $E$ is the entrainment rate.

The five unknowns in Eqn 3.37 and Eqn 3.38, are $\theta, \delta, \delta^{*}, C_{f}$, and $E$. The solution method for the boundary layer equations are arranged so that the two variables $\delta^{*}$ and $\Lambda$ where $\Lambda=\frac{\delta^{*}}{\delta}$ are unknowns and must be solved for. The remaining three unknowns, $\theta$, $C_{f}$, and $E$, are solved using correlations. The momentum thickness, $\theta$ can be expressed as

$$
\theta=\delta^{*}(1-\hbar)
$$

where $\hbar=f\left(\Lambda, M, \delta^{*}\right)$ is a shape factor. The skin friction coefficient is calculated by

$$
\frac{C_{f}}{2}=\kappa^{2} \Xi_{T}\left|\Xi_{T}\right|
$$

where $\kappa=0.41$ is the Von Karman constant and $\Xi_{T}=f\left(\Lambda, M, \delta^{*}\right)$ is a skin friction parameter. The entrainment rate correlation due to Bradshaw et al. [43] is used to determine $E$. Noting that $\hbar$ and $\Xi_{T}$ are functions of Mach number and the boundary layer equations Eqn. 3.37 and Eqn. 3.38 are expressed in terms of $U^{(\mathrm{comp})}$, the flow velocity can be expressed in terms of Mach number by

$$
U^{(c o m p)}=a M=a_{0} M\left(1+\frac{\gamma-1}{2} M^{2}\right)^{\frac{-1}{2}}
$$

The momentum and entrainment equations can be rewritten in terms of $\delta^{*}, \Lambda$, and $M$. The momentum equation, Eqn 3.37 written in terms of these variables is given by 
Childs [20] as

$$
\begin{aligned}
& (1-\hbar) \frac{d \delta^{*}}{d s}-\left[\delta^{*} \frac{\partial \hbar}{\partial \Lambda}\right] \frac{d \Lambda}{d s}+\left[\frac{\left(2-M^{2}\right)(1-\hbar)+1}{M\left(1+0.5(\gamma-1) M^{2}\right)}+\frac{\partial \hbar}{\partial M}\right] \delta^{*} \frac{d M}{d s} \\
& =\frac{C_{f}}{2}-(1-\hbar) \frac{\delta^{*}}{r} \frac{d r}{d s}-\frac{\partial \hbar}{\partial R e_{\delta}} \delta^{*} \frac{d R e_{\delta}}{d s}
\end{aligned}
$$

The entrainment equation, Eqn 3.38 written in terms of these variables is given below

$$
\begin{aligned}
& \left(\frac{1}{\Lambda}-1\right) \frac{d \delta^{*}}{d s}-\frac{\delta^{*}}{\Lambda^{2}} \frac{d \Lambda}{d s}+\left(\frac{1}{\Lambda}-1\right)\left[\frac{1-M^{2}}{1+0.5(\gamma-1) M^{2}}\right] \frac{\delta^{*}}{M} \frac{d M}{d s} \\
& =E-\left(\frac{1}{\Lambda}-1\right) \frac{\delta^{*}}{r} \frac{d r}{d s}
\end{aligned}
$$

Eqn 3.42 and Eqn 3.43 form a set of equations that can be solved simultaneously for the rate of change of $\delta^{*}$ and $\Lambda$ as the boundary layer is marched downstream.

The solution procedure for the boundary layer development along each intake surface is as follows. 1) Starting at the flow stagnation point, the intake surface is divided into several segments. 2) The surface Mach number and its first derivative at each surface location is calculated based on the inviscid flow solution. 3) The boundary layer is initially marched downstream using Thwaite's laminar boundary layer method [26] until laminarto-turbulent transition is predicted. Currently laminar-to-turbulent transition is assumed to occur at a momentum thickness based Reynolds number, $\operatorname{Re}_{\theta_{t r}}$, of 300 . This value of $R e_{\theta_{t r}}$ has been selected based on results from Dunham [44]. This simple transition criteria has been selected because the CFD software used in this thesis allows for the use of a simple $\operatorname{Re}_{\boldsymbol{\theta}_{t r}}$ based transition criteria. By using the same transition criteria for the design method and CFD simulations, differences in results due to transition criteria can be removed. However, simple transition criteria such as Michel's transition criteria can be implemented. Although Michel's transition criteria has also been implemented in this design method, all results presented here use the $\operatorname{Re}_{\theta_{t r}}$ based transition criteria. 4) The boundary layer is marched downstream from the laminar-to-turbulent transition 
point using Childs turbulent boundary layer method. The boundary layer parameters, $\delta^{*}$ and $\Lambda$ at the laminar-to-turbulent transition point are used as initial conditions for the Childs method. At each surface location downstream of the starting point, the system of equations, formed by the momentum and entrainment equations, is used to solve for the values of $\frac{d \delta^{*}}{d s}$ and $\frac{d \Lambda}{d s}$. The system of equations is shown below

$$
\left[\begin{array}{cc}
(1-\hbar) & -\left(\delta^{*} \frac{\partial \hbar}{\partial \Lambda}\right) \\
\left(\frac{1}{\Lambda}-1\right) & -\frac{\delta^{*}}{\Lambda^{2}}
\end{array}\right]\left[\begin{array}{c}
\frac{d \delta^{*}}{d s} \\
\frac{d \Lambda}{d s}
\end{array}\right]=\left[\begin{array}{c}
\omega_{1} \\
\omega_{2}
\end{array}\right]
$$

where $\omega_{1}$ and $\omega_{2}$ are

$$
\begin{aligned}
& \omega_{1}=-\left[\frac{\left(2-M^{2}\right)(1-\hbar)+1}{M\left(1+0.5(\gamma-1) M^{2}\right)}+\frac{\partial \hbar}{\partial M}\right] \delta^{*} \frac{d M}{d s}+\frac{C_{f}}{2}-(1-\hbar) \frac{\delta^{*}}{r} \frac{d r}{d s}-\frac{\partial \hbar}{\partial R e_{\delta}} \delta^{*} \frac{d R e_{\delta}}{d s} \\
& \omega_{2}=-\left(\frac{1}{\Lambda}-1\right)\left[\frac{1-M^{2}}{1+0.5(\gamma-1) M^{2}}\right] \frac{\delta^{*}}{M} \frac{d M}{d s}+E-\left(\frac{1}{\Lambda}-1\right) \frac{\delta^{*}}{r} \frac{d r}{d s}
\end{aligned}
$$

5) The Runge-Kutta method is used along with the calculated values of $\frac{d \delta^{*}}{d s}$ and $\frac{d \Lambda}{d s}$ to propagate the values of $\delta^{*}$ and $\Lambda$ downstream to the next surface location. 6) Steps 4-5 are repeated until either the boundary layer reaches the end of the intake surface, or separation is detected.

Boundary layer separation is predicted by Childs method when the boundary layer parameter $\Lambda$ equals 0.42 since this value of $\Lambda$ has been shown by Bardina et al. [45] to correspond to incipient boundary layer detachment in 2D diffusers. Past separation point, the boundary layer is assumed to extend straight downstream parallel to the z-axis for the purpose of calculating the viscous flow area, $A_{3}^{(\text {visc })}$.

Because Childs' boundary layer method is only valid up to Mach numbers of 1, a modification must be made to the boundary layer calculations in regions of supersonic flow. Remembering that the flow analysis in this work is restricted to subsonic freestream velocities, regions of supersonic flow can occur around the cowl lip while the intake is 
operating at large $F C$ ratios, or along the centre body in region $\mathrm{A}$ and region $\mathrm{B}$ as the freestream Mach number approaches $M=1$. In situations where local Mach numbers are greater than 1 , it is assumed that a separation bubble forms in the supersonic region and the boundary layer reattaches downstream of the supersonic region. The boundary layer is marched up to the the beginning of the supersonic flow region using Child's method. The boundary layer calculations are then restarted at the end of the supersonic region with values of $\delta^{*}$ and $\Lambda$ which are twice that of their values at the beginning of the supersonic flow region. The magnitude of the change in $\delta^{*}$ and $\Lambda$ is based on results presented by Seddon [18] for the interaction between a boundary layer and a normal shock at an initial Mach number of 1.47. The assumptions made in this treatment of the boundary layer behaviour through a shock limits its validity to simple, weak shocks. Implementation of this boundary layer treatment into the design method has been found to be stable for some intake geometries, but is unstable (the boundary layer grows rapidly and blocks flow) for other geometries. Therefore, more work is required to develop a stable method of dealing with these flow situations.

For the flight conditions used in this work, none of the air intake geometries contain any regions of supersonic flow, and therefore the methods described in the previous paragraph are not used.

To summarize the flow analysis to this point, the inviscid flow solver is used to obtain the compressible, inviscid surface velocities along the air intake surfaces for an intake operating at a given set of flight conditions. The inviscid surface velocities are then used as an input into the boundary layer method of the viscous flow solver. The boundary layer calculations output the value of $\delta^{*}, \Lambda$, and $C_{f}$ along the intake surfaces. The value of $\delta^{*}$ at plane 3 for all intake surfaces is then used to calculate the corrected flow area at plane $3, A_{3}^{(v i s c)}$, which is then used to calculate mass flow rate (See Eqn. 3.1). In cases where the boundary layer has separated, $A_{3}^{(v i s c)}$ is calculated based on the intersection of plane 3 and the separated boundary layer which is extended straight downstream from the separation 
point.

The following section details the calculation of total pressure losses, which leads to the calculation of the remaining variables in Eqn. 3.1.

\subsubsection{Total Pressure Losses}

The viscous flow solver estimates viscous losses by estimating total pressure losses in the intake flow. Since the static pressure at plane $3, p_{3}$, is assumed to be fixed, any reduction in total pressure reduces $M_{3}$, and thus reduces $m$. The method used to estimate total pressure losses is that of flow through a constant area duct with friction and assumes the flow streamtube is bounded or partially bounded by no-slip surfaces and that the streamtube walls are not significantly inclined from the streamtube centre line. This method assumes total pressure losses are due solely to friction (i.e. it does not account for losses due to flow separation). This assumption is made based on the idea that the air intake geometry is designed to avoid large regions of flow separation. The friction force, $F_{f}$, on an element along the streamtube walls, shown in figure 3.13 , is equal to

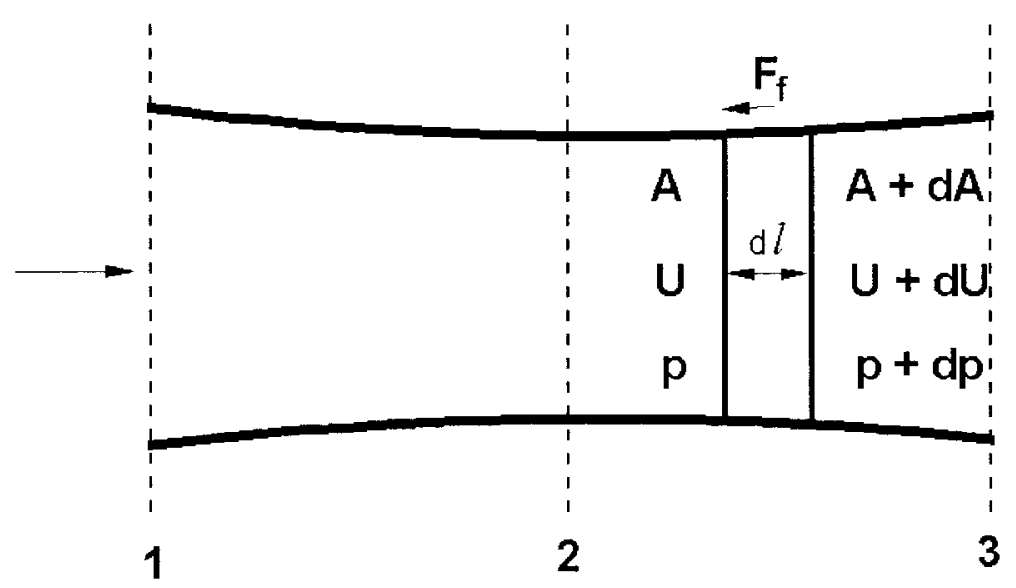

Figure 3.13: Streamtube with no-slip walls and streamtube element 


$$
F_{f}=q C_{f} \zeta d \ell
$$

where $q$ is the local dynamic pressure, $C_{f}$ is the local skin friction coefficient found from the boundary layer calculations, and $\zeta$ is the local perimeter length of the streamtube element. Since the boundary layer calculations have been performed on each of the three intake surfaces, there will be three different $C_{f}$ and $\zeta$ distributions and thus the total pressure loss due to each surface must be calculated.

The general total pressure loss calculation for a surface begins by applying the momentum equation to the streamtube element in figure 3.13 and gives

$$
p A-(p+d p) A-F_{f}=\dot{m}(U+d U-U)
$$

Remembering that $\dot{m}=\rho A U$, and assuming incompressible flow, Eqn. 3.47 reduces to

$$
d p+\rho U d U=\frac{-F_{f}}{A}
$$

Eqn. 3.48 can then be written as

$$
d\left(p+\frac{1}{2} \rho U^{2}\right)=d P_{0}=-\frac{F_{f}}{A}
$$

Eqn. 3.49 relates the friction force on a streamtube element directly to the change in total pressure of the element. The loss in total pressure over the entire streamtube can be estimated by integrating Eqn. 3.49 along the streamtube length using the equation

$$
\Delta P_{0}=\int_{1}^{3} \frac{F_{f}}{A}=\int_{1}^{3} q C_{f} \frac{\zeta}{A} d \ell
$$

where the local density and velocity found from the inviscid flow solution are used to 
calculate the local dynamic pressure $q$.

The complete total pressure loss through the air intake is calculated by dividing each intake surface into a large number of segments. The local value of the integrand of Eqn. 3.50 at each segment is calculated using the local values of $C_{f}, \rho^{(\operatorname{comp})}, U^{(\operatorname{comp})}$ and $A$, which are calculated from the inviscid flow field and the boundary layer data. This data is then numerically integrated to find the total pressure loss due to the friction along each surface. Finally, the combined total pressure loss through the intake due to all three intake surfaces is found by summing the individual total pressure losses from each surface as is illustrated in figure 3.14 .

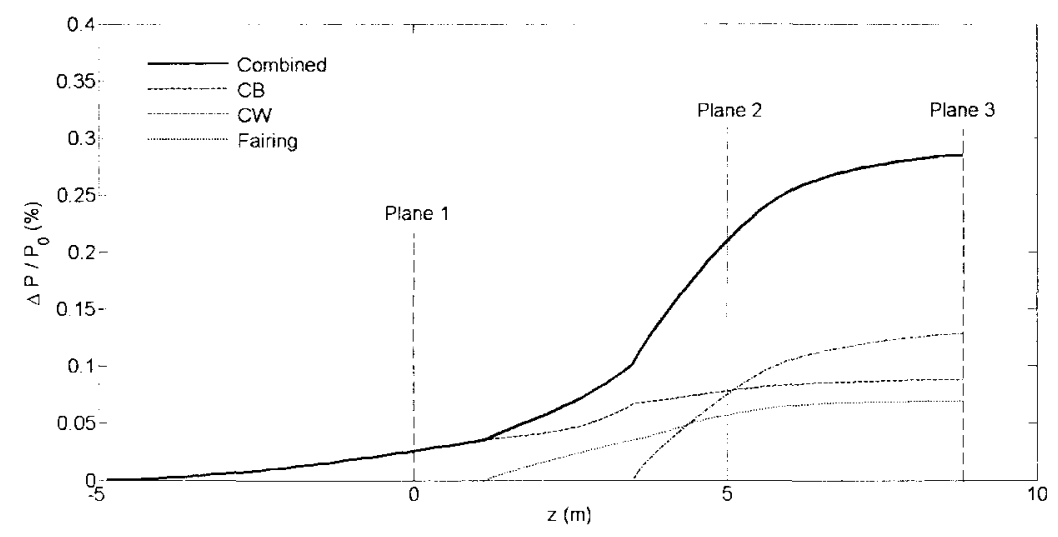

Figure 3.14: Combined total pressure loss along air intake with breakdown of total pressure loss due to each intake surface.

\subsection{Air Mass Flow Rate Calculation}

The final step in the flow analysis is to estimate the air mass flow rate through the intake. Recalling Eqn. 3.1 the mass flow rate is a function of the values of $A_{3}, \rho_{3}$, and $U_{z_{3}}$, corrected for viscous effects.

The value of $A_{3}^{(v i s c)}$ is calculated from the intake geometry and the $\delta^{*}$ data from boundary layer calculations along each of the intake bodies. For intake geometries where the 
fairing geometry intersects plane 3, the area reduction due to the fairing geometry is also accounted for. The area calculation is carried out in the same manner as the plane 2 and plane 3 area calculations described in Section 2.2, except the radial boundaries are adjusted using the $\delta^{*}$ data.

The values of $\rho_{3}^{(v i s c)}$, and $U_{z_{3}}^{(v i s c)}$ are calculated using the total pressure loss results. Because $p_{3}$ is fixed, the viscous density and velocity at plane 3 can be determined based on the corrected total pressure at plane 3 . The corrected total pressure at plane 3 is simply

$$
P_{0_{3}}^{(v i s c)}=P_{0}-\Delta P_{0}
$$

Using the isentropic flow relations for air, the corrected Mach number at plane 3 is then

$$
M_{3}^{(v i s c)}=\left[\frac{2}{\gamma-1}\left({\frac{P_{0_{3}}^{(v i s c) \frac{\gamma-1}{\gamma}}}{p_{3}}}^{\frac{\gamma}{2}}\right)\right]^{\frac{1}{2}}
$$

Assuming adiabatic flow so that $a_{0}$ is constant and using Eqn. 3.41 the corrected flow velocity at plane 3 is then

$$
U_{z_{3}}^{(v i s c)}=a_{0} M_{3}^{(v i s c)}\left(\left(1+\frac{\gamma-1}{2} M_{3}^{(v i s c) 2}\right)^{\frac{-1}{2}}\right)
$$

In order to calculate the corrected density at plane 3, the total density at plane 3 must first be calculated since the total pressure at plane 3 has been adjusted. Using the perfect gas law (where $T_{0}$ is constant), the total density at plane 3 is

$$
\rho_{0_{3}}^{(v i s c)}=\rho_{0} \frac{P_{0_{3}}^{(\text {visc })}}{P_{0}}
$$


And the corrected density at plane 3 is

$$
\rho_{3}^{(v i s c)}=\rho_{0_{3}}^{(v i s c)}\left[\left(1+\frac{\gamma-1}{2} M_{3}^{(v i s c) 2}\right)^{\frac{-1}{\gamma-1}}\right]
$$

With the values of $A_{3}^{(v i s c)}, \rho_{3}^{(v i s c)}$, and $U_{z_{3}}^{(v i s c)}$ calculated, the air intake mass flow rate is calculated according to Eqn. 3.1. In addition to evaluating an air intake geometry based on the actual mass flow rate through the intake, it can also be useful to look at the loss in mass flow rate and flow area due to viscous effects. The parameter $\Upsilon_{A}$ describes the percent loss in flow area due to boundary layer blockage and is defined as

$$
\Upsilon_{A}=\frac{A_{3}-A_{3}^{(v i s c)}}{A_{3}}
$$

The lower the value of $\Upsilon_{A}$, the smaller the viscous losses, with $\Upsilon_{A}=0$ being an inviscid flow solution. Similarly, the parameter $\Upsilon_{m}$ describes the percent loss in mass flow rate due to viscous effects, and is defined as

$$
\Upsilon_{m}=\frac{\left(\bar{\rho}_{3} A_{3} \bar{U}_{z_{3}}\right)-\left(\bar{\rho}_{3} A_{3} \bar{U}_{z_{3}}\right)^{(v i s c)}}{\left(\bar{\rho}_{3} A_{3} \bar{U}_{z_{3}}\right)}
$$

\subsection{Flow Analysis Panel Sensitivity}

A consideration when conducting the flow analysis on a particular intake geometry is how many surface panels are required to obtain a panel independent solution. Therefore, a panel sensitivity study has been conducted on an air intake geometry operating at 3 different points along the Ariane 4 flight profile, shown in figure 3.1. While the exact number of panels required for a panel independent solution will be specific to each intake geometry, the results from the panel sensitivity study will give the user a good initial guess at the 


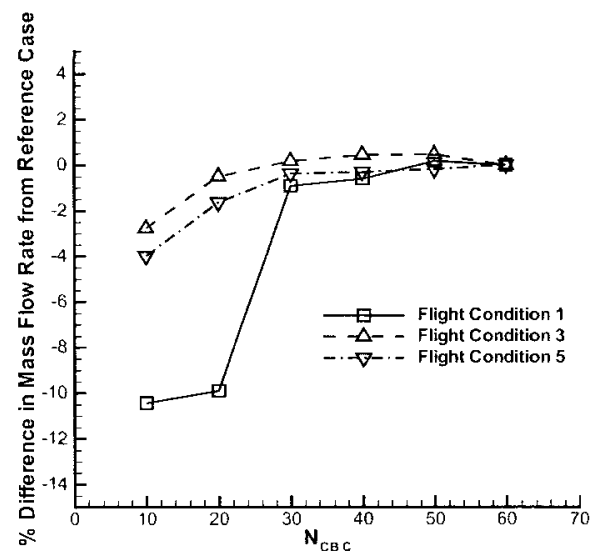

(a) Mass flow rate percent difference from reference vs. Number of panels in centre body region C.

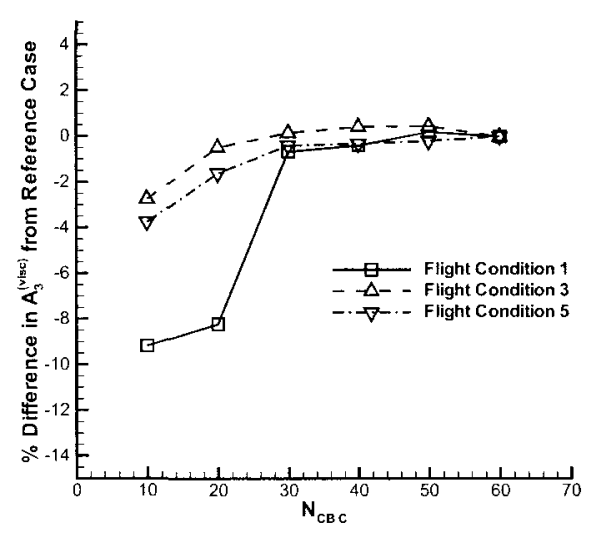

(b) $A_{3}^{(\text {visc })}$ percent difference from reference vs. Number of panels in centre body region $C$.

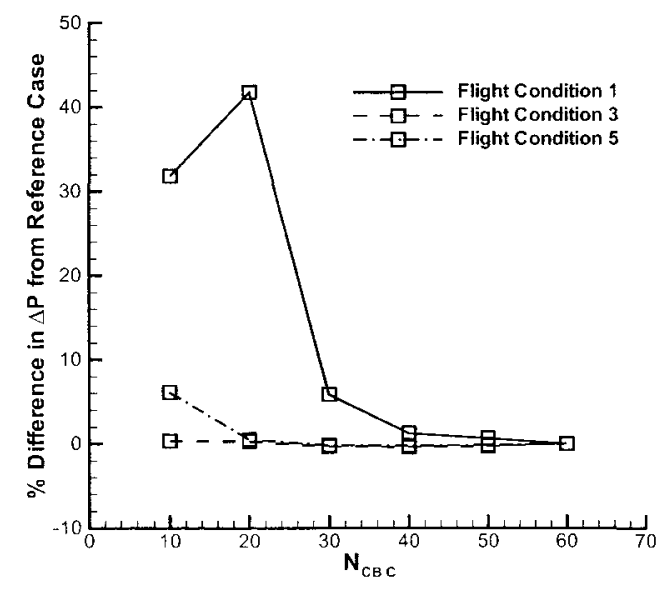

(c) $\Delta P$ percent difference from reference vs. Number of panels in centre body region $C$.

Figure 3.15: Results from panel sensitivity study.

number of panels required. The sensitivity of $\dot{m}, A_{3}^{(v i s c)}$, and $\triangle P_{0}$ to $N_{C B C}$ are shown in figures 3.15(a)-(c). The sensitivity of $\triangle P$ to $N_{C B C}$ is chosen because it affects both $\rho_{3}^{(v i s c)}$ and $U_{z 3}^{(v i s c)}$, which in turn affect $\dot{m}$. The results have been referenced to the $N_{C B C}=60$ case and the percent difference from the reference case for each parameter, at each flight condition is plotted with respect to the number of panels used in centre body region $\mathrm{C}$. 
A situation where the design method results can be very sensitive to panel density is where the intake is operating at a large flow capture ratio, such as at flight condition 1 , and the boundary layer is very close to separating. In these situations, the flow must turn around the cowl lip where it is accelerated to large peak velocities and then rapidly decelerated as it enters the duct. Because this velocity spike occurs over a relatively small distance, the shape of this velocity spike tends to change as the number of panels increases. After a certain point, as the number of panels increases, the shape of the surface velocity profile begins to converge. As the surface velocity profiles begin to converge, shown in figure 3.16 , the values of the output parameters also begin to converge.

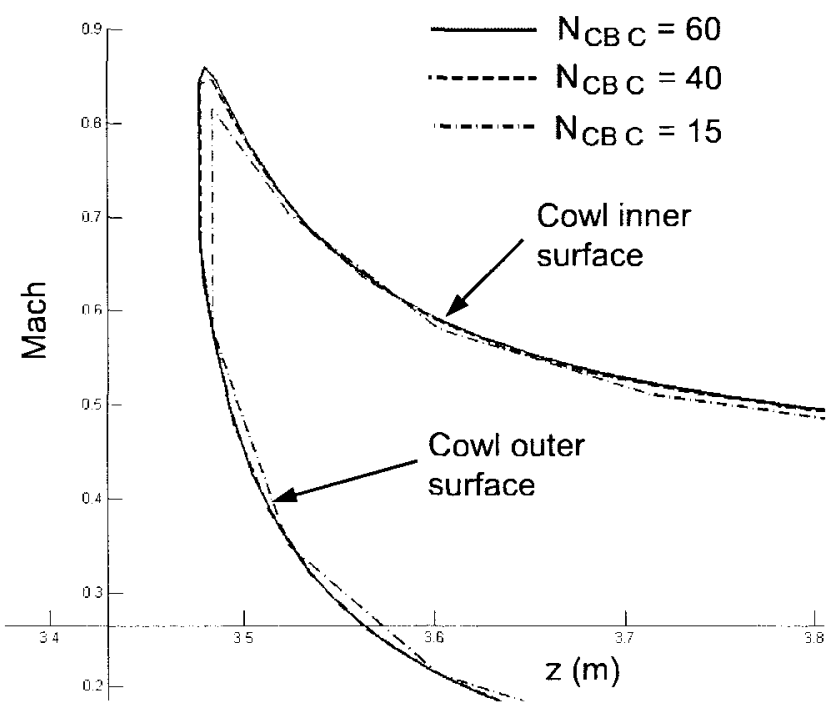

Figure 3.16: Mach number distribution along inner and outer cowl leading edge surfaces using various panel resolutions.

The results from the panel sensitivity study indicate that above $N_{C B C}=40$, the flow analysis results converge with a variation of approximately than $1 \%$ for each flight condition. Therefore, a value of $N_{C B C} \geq 40$ is recommended for the flow analysis. 


\subsection{Flow Analysis Validation}

In order to confirm that the flow analysis method produces results which follow physically observed trends, the flow analysis results have been compared to some general intake experimental results.

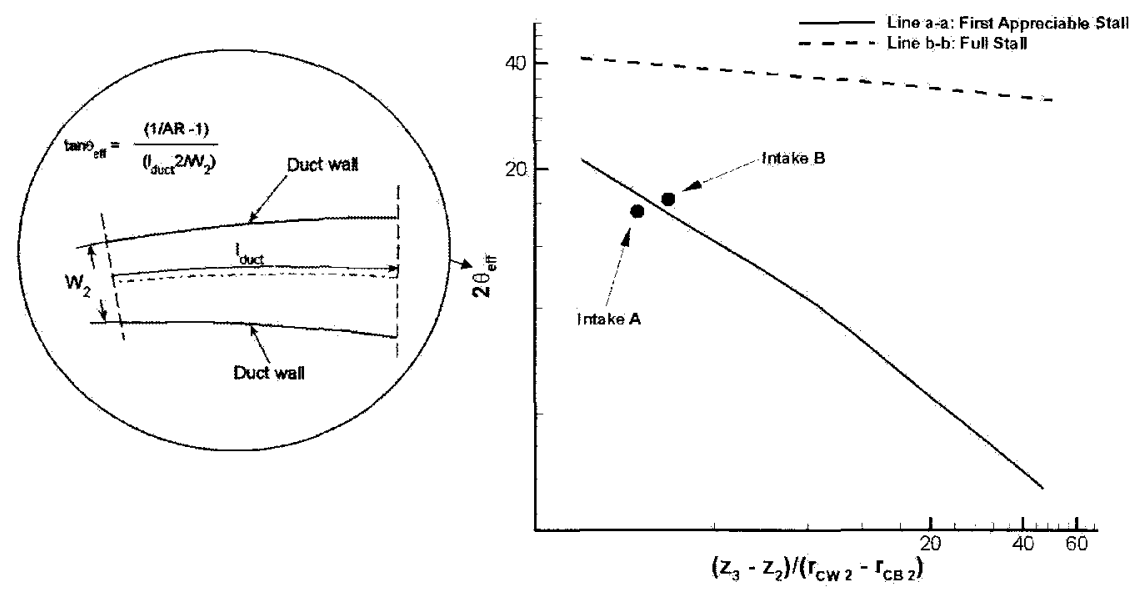

Figure 3.17: Fox-Kline [46] curved subsonic diffuser flow regime plot with performance points for two air intake geometries. Definition of $\theta_{\text {eff }}$ is also shown on figure.

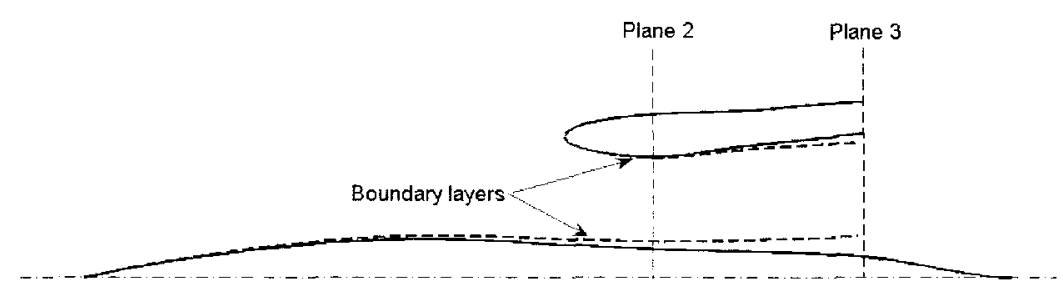

(a) Intake geometry A with boundary layer.

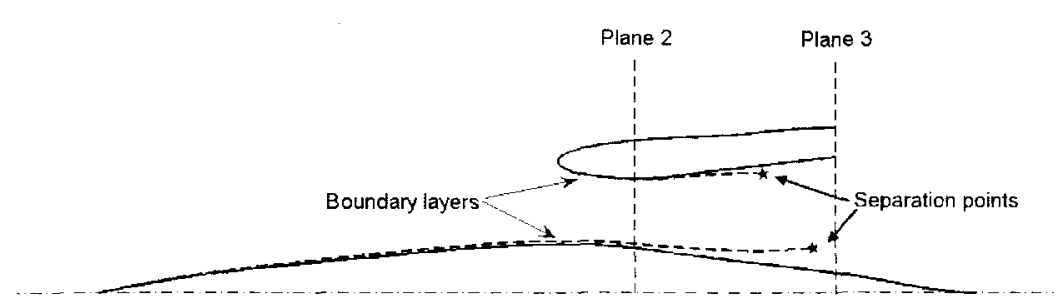

(b) Intake geometry B with boundary layer.

Figure 3.18: Intake geometries from Fox-Kline plot. 
Fox and Kline [46] developed a chart for predicting the types of flow regimes a particular subsonic intake is likely to experience based on its geometry. More specifically, the Fox-Kline plot correlates the expected flow regime to the intakes $A R$ and length. The plot shown in figure 3.17 is for subsonic diffuers with curved centre-lines. The two lines on figure 3.17 indicate the boundaries where the intake flow is expected to transition between different flow regimes. An intake geometry which lies to the left of line a-a would be expected to contain fully attached flow, and an intake geometry which lies to the right of line a-a would be expected to contain small separated flow regions. The flow analysis has been conducted on two air intake geometries which lie on either side of line a-a, shown in figure 3.17. The operating point of the intakes has been selected such that the flow remains essentially incompressible $(M \leq 0.3)$ since the experiments by Fox and Kline [46] were incompressible. Intake geometry $\mathrm{A}$, which lies on the left of line a-a does not contain any regions of separated flow, as shown in figure 3.18(a), and intake geometry B contains small regions of separated flow, shown in figure 3.18(b). These results are consistent with the Fox and Kline experiments.

An important feature of air intakes is the cowl lip geometry. The cowl lip affects the flow conditions over which the intake can operate without significant total pressure losses. The lip contraction ratio, $C R$, in particular affects the intake performance at the static flow condition, where the freestream velocity is close to zero and the intake mass flow rate is non-zero. In this situation the flow stagnates on the outside of the cowl, as illustrated in figure 3.19 , and the flow must turn around the cowl lip to enter the intake. As the flow turns around the lip it experiences large peak velocities, which can lead to flow separation. Seddon [18] presents a plot, shown in figure 3.20, of $M_{\text {peak }} / M_{2}$ with respect to $C R$ using a potential flow method. The design method results for several values of $C R$ are also plotted in figure 3.20 for comparison. The design method results follow the same trends as the results by Seddon [18] but are offset slightly. This offset is likely due to differences in the cowl lip geometry since the cowl lip geometry is not a pure elliptical profile, as used by 
Seddon, due to the blend regions, and the intake used by Seddon did not contain a centre body.

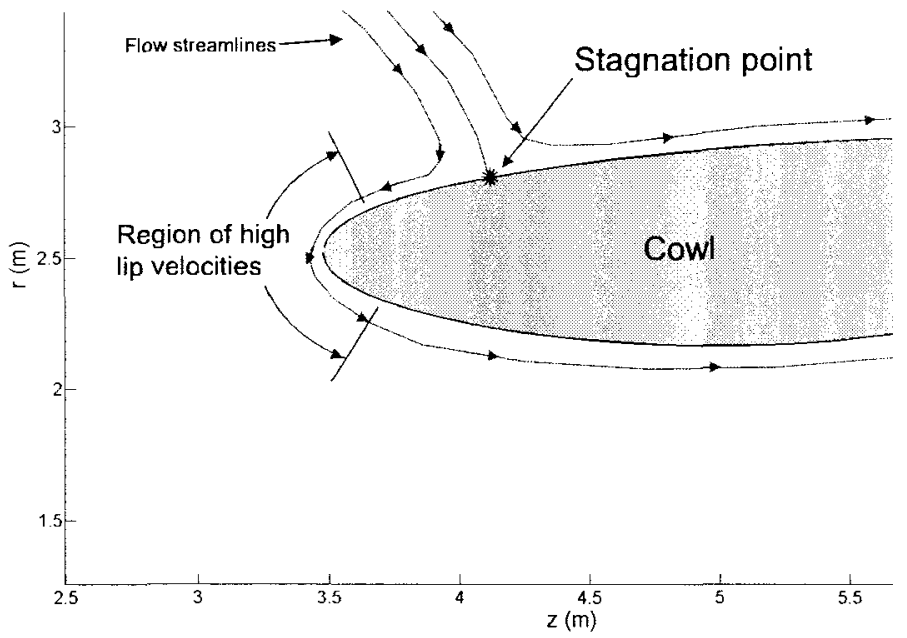

Figure 3.19: Cowl lip with flow stagnating on outer cowl surface.

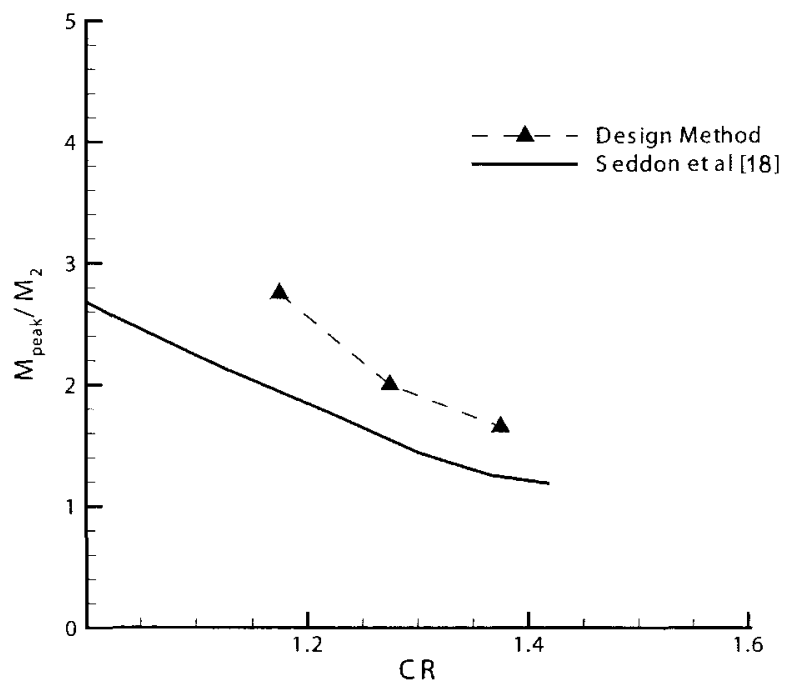

Figure 3.20: Plot of $M_{\text {peak }} / M_{2}$ vs. $C R$ using potential flow methods by Seddon [18] with intake design method results.

The results presented in this section give one some confidence that the flow analysis method used here produces results which follow the same trends as physical experiments. 
In addition, the flow analysis method used in this work is more versatile than the ruleof-thumb plots such as the Fox-Kline plot since these charts only consider a few aspects of the intake geometry and flow conditions. For example, a flow analysis at several flight conditions has been conducted on two intake geometries, Intake geometry $\mathrm{C}$ and $\mathrm{D}$, shown in figure 3.21, which lie in the fully attached flow region of the Fox-Kline plot. The only difference between these geometries are the values of $C R$ and $\tilde{t}_{3}$. Intake geometry $\mathrm{D}$ has a smaller value of $C R$ and a larger value of $\tilde{t}_{3}$ so that the centre body radius at plane 3 is smaller than that of geometry $C$. The values of $\Upsilon_{A}$ for the flight conditions shown in figure 3.1 are plotted on figure 3.22. The results show that intake geometry D, with its lower value of $C R$, experiences larger peak velocities around the cowl lip and as a result, flow separation off of the cowl lip at flow condition 1. The early separation along the cowl lip for intake geometry $D$ means the value of $\Upsilon_{A}$ is greatly increased relative to intake geometry C. At flight condition 5, the high speed flow condition, due to the smaller value of $r_{C B_{3}}$ intake geometry $\mathrm{D}$ experiences earlier flow separation along the centre body, and thus has a larger $\Upsilon_{A}$ relative to geometry $C$. These differences in intake performance would not have been predicted by the Fox-Kline plot. 


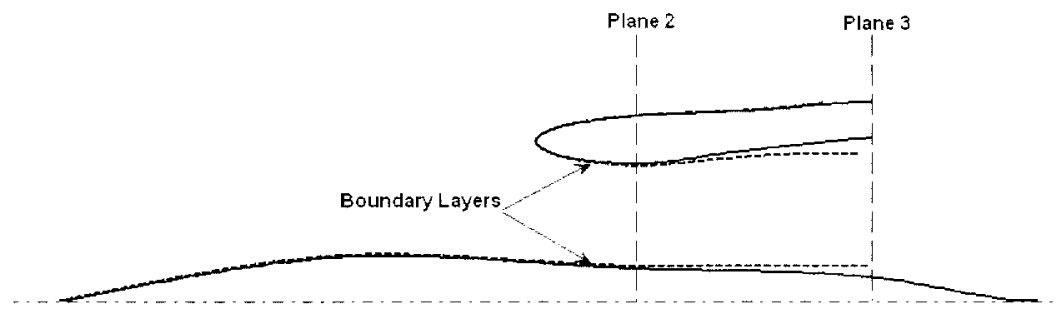

(a) Intake geometry $\mathrm{C}$ operating at flight condition 1 with boundary layer shown.

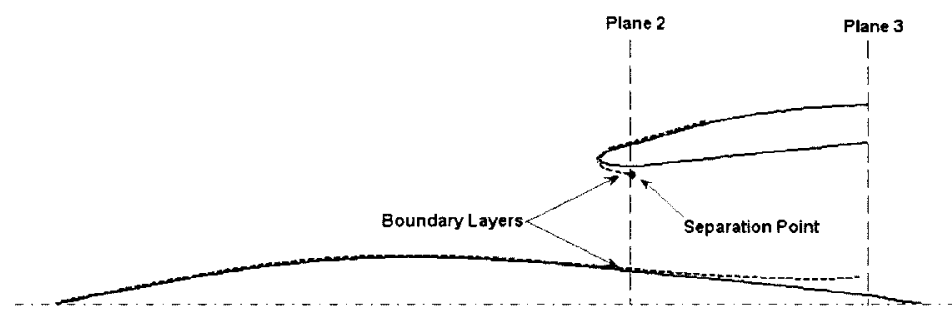

(b) Intake geometry D operating at flight condition 1 with boundary layer shown.

Figure 3.21: Intake geometries $\mathrm{C}$ and $\mathrm{D}$ with boundary layer shown for flight condition 1 .

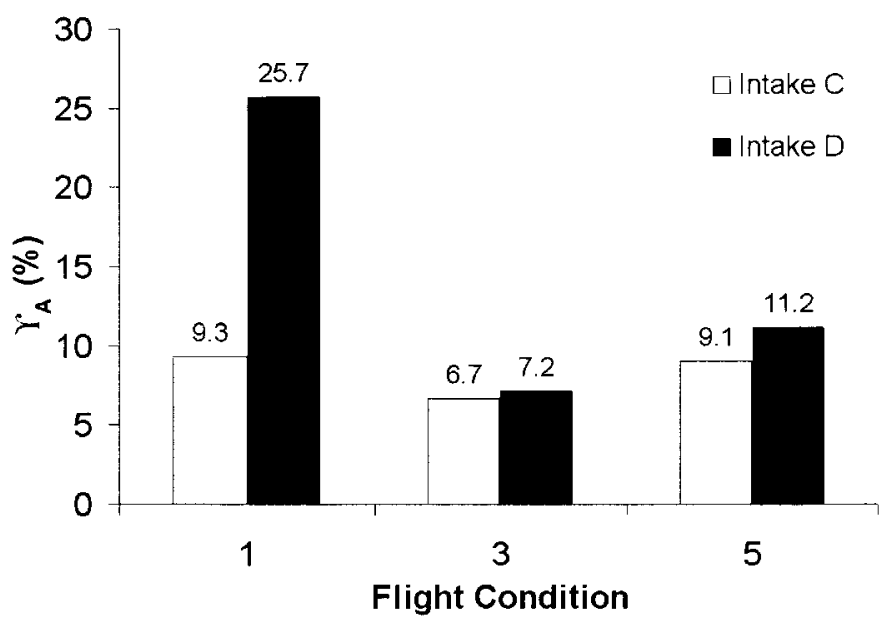

Figure 3.22: $\Upsilon_{A}$ for intake geometries $C$ and $D$ for 3 flight conditions. 


\section{Chapter 4}

\section{Air Intake Design Method Case Study}

This chapter presents a practical example of how the air intake design method described in the previous chapters can be used to design a rocket nozzle and air intake for an RBCC vehicle. The design method described has been implemented using MATLAB ${ }^{\circledR}$ 7.0.1 and has a run time of approximately 2 minutes using a $2.4 \mathrm{GHz}$ processor. The following sections will detail the flight profile selection, geometry selection procedure, and the flow analysis results.

\subsection{Rocket Nozzle Geometry Selection}

For this case study, the rocket nozzle, designed using the method developed by Cerantola and Etele $[16,17]$, has been designed to reproduce the performance of the LE-7A rocket engine. This rocket engine is used in the first stage of the H-IIA launch vehicle, which is operated by the Japanese Aerospace Exploration Agency (JAXA). The specifications for the LE-7A liquid $O_{2} /$ liquid $H_{2}$ rocket engine are provided in table 4.1. 
Table 4.1: LE-7A Specifications [47]

\begin{tabular}{cc} 
Specification & Value \\
\hline Thrust(vacuum) & $870 \mathrm{kN}(1098 \mathrm{kN})$ \\
Isp(vacuum) & $338 \mathrm{~s}(440 \mathrm{~s})$ \\
Chamber Pressure & $1.21 \mathrm{E} 04 \mathrm{kPa}(121.00 \mathrm{bar})$ \\
Area Ratio & 51.90 \\
Oxidizer to Fuel Ratio & 5.90 \\
Length & $3.67 \mathrm{~m}$ \\
Mass Flow Rate & $247 \mathrm{~kg} / \mathrm{s}$
\end{tabular}

Table 4.2: Flight Profile Breakdown

\begin{tabular}{c|ccccc} 
Flight Profile Point & 1 & 2 & 3 & 4 & 5 \\
\hline$M_{\infty}$ & 0.01 & 0.19 & 0.41 & 0.64 & 0.89 \\
$h_{A / t}(\mathrm{~m})$ & 40 & 929 & 2196 & 3800 & 5741 \\
$p_{3}(\mathrm{kPa})$ & 97.7 & 89.2 & 83.1 & 79.1 & 77.6
\end{tabular}

\subsection{Flight Profile Selection and Rocket-Ejector Configu- ration}

The flight profile selected for this case study is an approximation of the Ariane 4 flight profile, shown in figure 3.1. This profile was selected since it represents an actual launch vehicle flight profile and data is readily available through Turner [39]. The flow analysis is conducted at 5 points along this flight profile. The $M_{\infty}$ and $h_{A l t}$ at these 5 points are listed in table 4.2. With the flight profile defined, an ejector section geometry must be selected in order to determine the value of $\sigma$ required for the air intake, as well as the value of $p_{3}$ for the flow analysis. The value of $\sigma$, which is the ratio between the rocket exhaust 
flow area and the total flow area (See Table 2.1), is required to fully define the air intake geometry, as well as to determine a value of $p_{3}$ for each flight condition. The rocket-ejector analysis method developed by Etele et al. [8] has been used here to select the rocketejector configuration for the RBCC vehicle. The rocket-ejector analysis method takes the fixed rocket engine performance parameters, flight conditions $\left(P_{0_{\infty}}, T_{0_{\infty}}, M_{\infty}\right), \sigma$, the mixed flow static pressure in the ejector section, $p_{\text {Mixed }}$ (which assumes a subsonic mixed flow), and the ejector section nozzle geometry as inputs and outputs the thrust augmentation ratio. Figure 4.1 shows a diagram of the general rocket-ejector configuration. The thrust augmentation ratio, $\Phi$, is calculated as follows

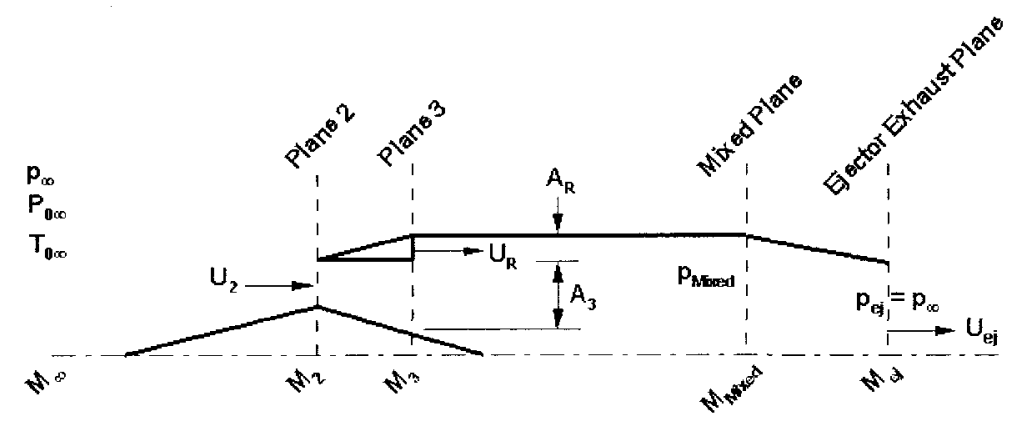

Figure 4.1: RBCC rocket ejector engine diagram.

$$
\Phi=\frac{A_{e j}\left(p_{e j}-p_{\infty}\right)-A_{2}\left(p_{2}-p_{\infty}\right)+\dot{m}_{R}\left[(\alpha+1) U_{e j}-\alpha U_{2}\right]}{F_{R}}
$$

where $\alpha$ is the ratio between the air and rocket exhaust mass flow rates, the subscript $e_{j}$ refers to the ejector exhaust plane, and $F_{R}$ is the thrust of the rocket engine alone. The selection procedure for the rocket-ejector configuration begins by first choosing an ejector section nozzle geometry and value of $\sigma$. The mixed flow pressure is then varied over the 5 flight conditions such that the ejector nozzle exit pressure is as close to the freestream pressure as possible for a fixed ejector nozzle geometry. Several different combinations of the rocket-ejector analysis input parameters are analyzed. The final rocket-ejector configuration was chosen such that the mission averaged value of $\Phi \geq 1$ for the 5 flight conditions. 
Because optimization of a rocket-ejector configuration for a given flight profile is not the primary goal of this work, the rocket-ejector configuration used here does not necessarily represent an ideal configuration but should yield improved performance based on a mission averaged value of $\Phi \geq 1$. The final rocket-ejector configuration used in this work

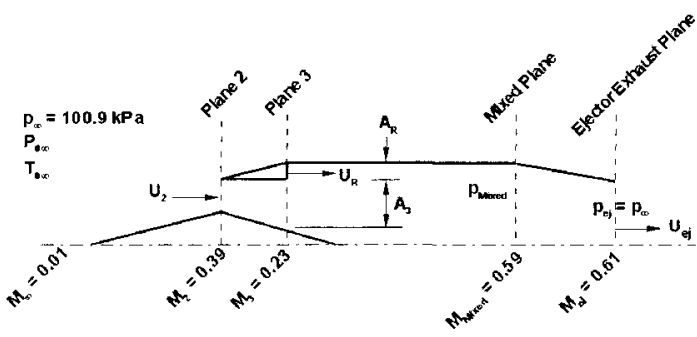

(a) Flight condition 1.

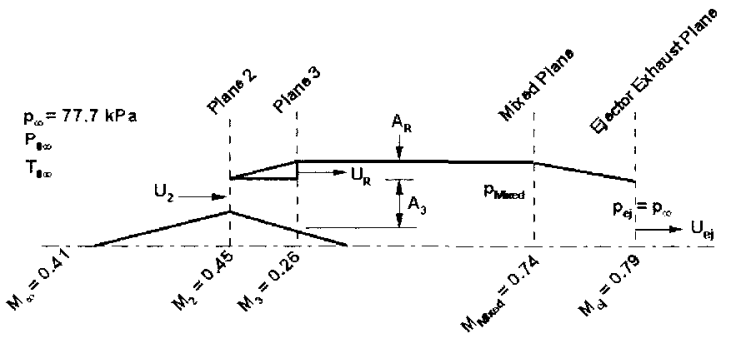

(c) Flight condition 3.

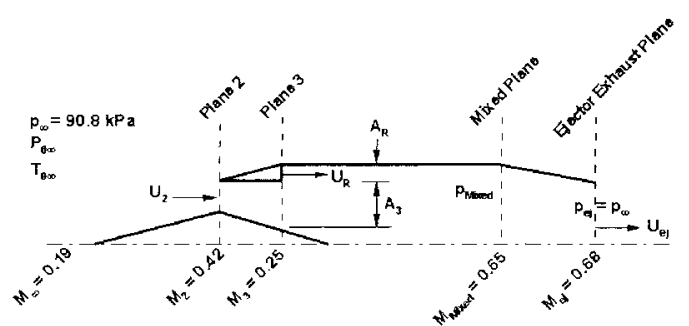

(b) Flight condition 2 .

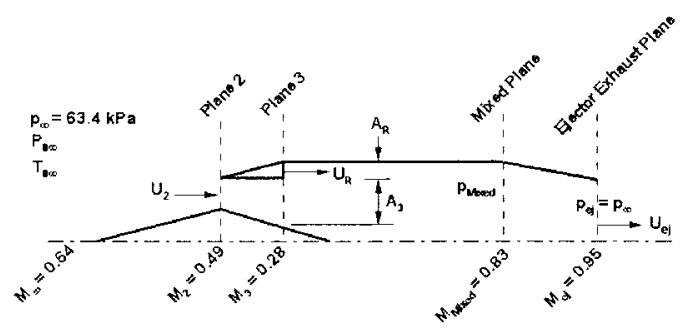

(d) Flight condition 4 .

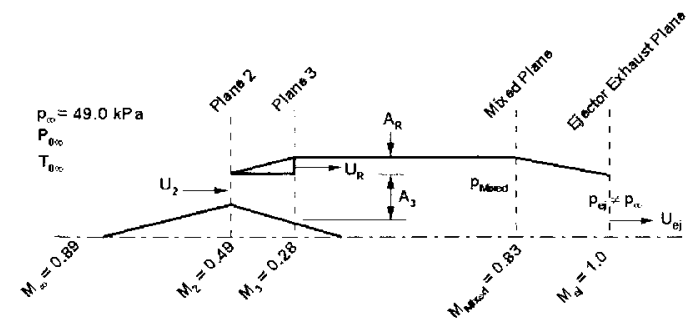

(e) Flight condition 5 .

Figure 4.2: RBCC rocket-ejector engine diagrams for 1-5 flight conditions.

has $\sigma=0.1$ and the values of $p_{3}$ for each flight condition are provided in table 4.2. The average thrust augmentation ratio for this rocket-ejector configuration over flight conditions 1 to 5 is $\bar{\Phi}=1.067$. This means a fixed geometry rocket-ejector engine as shown in figure 4.1 will produce approximately $7 \%$ more thrust than the rocket in isolation over the same launch profile, provided the rocket-ejector can achieve the air entrainment ratio 
as predicted in the rocket-ejector analysis. The flow Mach numbers through the RBCC engine when operating at the 5 flight conditions is shown in figure 4.2.

\subsection{Geometry Selection}

The final geometry has been selected with the goal of maintaining attached flow through the air intake (between plane 2 and plane 3) for flight conditions 2 and 3. The selected geometry is predicted to see regions of flow separation at flight conditions 1,4 and 5 . The location of flow separation will be compared to the flow separation location in CFD simulations to determine the accuracy of the flow separation criteria used by the flow analysis. The input parameters required to generate the selected geometry are listed in table 4.3 while figure 4.4 shows the geometry along symmetry plane 1 .

The design method flow analysis results for $\alpha\left(\alpha=\dot{m} / \dot{m}_{R}\right), \Upsilon_{A}$ and $\Upsilon_{m}$ are plotted in figure 4.3(a)-(b). The parameters $\Upsilon_{A}$ and $\Upsilon_{m}$ are defined in section 3.4 and describe the percent loss, due to viscous effects, of flow area and mass flow rate respectively. Figure 4.3(a) shows the viscous and inviscid values of $\alpha$. The inviscid values of $\alpha$ are obtained from the rocket-ejector code and represent the ideal value of $\alpha$ with no viscous losses and complete mixing of the rocket and entrained air streams. While the inviscid value of $\alpha$ decreases between flight conditions $1-3$, the viscous $\alpha$ results show an increase between flight conditions $1-2$. This increase in $\alpha$, which means the intake viscous mass flow rate at flight condition 1 is lower than at flight condition 2, corresponds to the $\Upsilon_{A}$ and $\Upsilon_{m}$ in figure 4.3(b). The high values of $\Upsilon_{A}$ and $\Upsilon_{m}$ at flight condition 1 are due to the thick boundary layer along the cowl surface as well as boundary layer separation along the cowl surface. Overall, figure 4.3(b) shows that the $\Upsilon_{A}$ and $\Upsilon_{m}$ results follow similar trends between flight conditions $1-5$. The value of $\Upsilon_{m}$ is at a minimum at flight condition 2 , indicating that viscous losses are at a minimum around this flight condition, beyond this flight condition, viscous losses continue to increase. For flight conditions 4 and $5, \Upsilon_{A}$ and 
Table 4.3: Selected Air Intake Geometry

\begin{tabular}{c|c} 
Geometry Parameter & Value \\
\hline$\sigma$ & 0.10 \\
$\tilde{z}_{2}$ & 0.425 \\
$A R$ & 0.625 \\
$\tilde{r}_{C B_{1}}$ & 5.5 \\
$C R$ & 1.35 \\
$\tilde{t}_{3}$ & 0.13 \\
$\tilde{c}_{f a i r_{\max }}$ & 1.115 \\
$A_{2-3}$ & Linear
\end{tabular}

$\Upsilon_{m}$ begins to level off. At flight conditions 4 and 5 flow separation is predicted along the centre body surface, shown in figure 4.4 for flight condition 4 . The separation location for flight condition 5 is further upstream than at condition 4 , yet $\Upsilon_{A}$ only increases by $1.4 \%$ between the two flight conditions. This small change in $\Upsilon_{A}$ is due to the centre body shape having a more or less constant radius through a large portion of region $\mathrm{C}$ which when combined with the fact that after separation the boundary layer is assumed to remain at at constant radius, yields very little change in $A_{3}^{(\text {visc })}$.

Using the $\Upsilon_{A}$ results from the flow analysis, the rocket-ejector thrust calculations were carried out using adjusted ejector intake flow areas in order to reflect the reduced entrainment effect. A reduction in air entrainment effectively increases the value of $\sigma$. Using the adjusted values of $\sigma$, the average thrust augmentation over the 5 flight conditions for this air intake geometry is $\Phi=0.95$. These results indicate that using this particular air intake geometry and the Ariane 4 flight profile, average thrust is reduced by $5 \%$. Therefore, further optimization of the air intake geometry is recommended.

While performance of the rocket-ejector and air intake configuration used here over the Ariane 4 flight profile would suggest that the particular air intake geometry used in this 


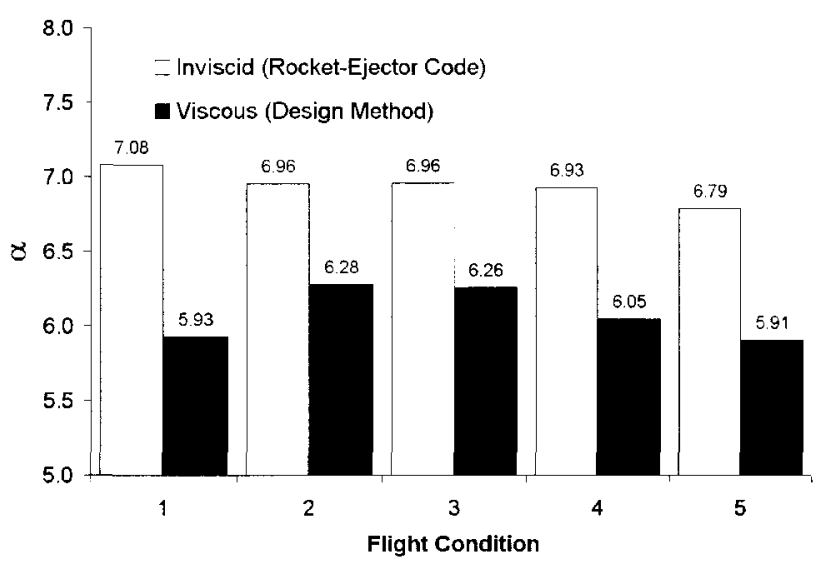

(a) $\alpha$ for the selected intake geometry over 5 flight conditions. $\alpha=\frac{\dot{m}}{\dot{m}_{R}}$

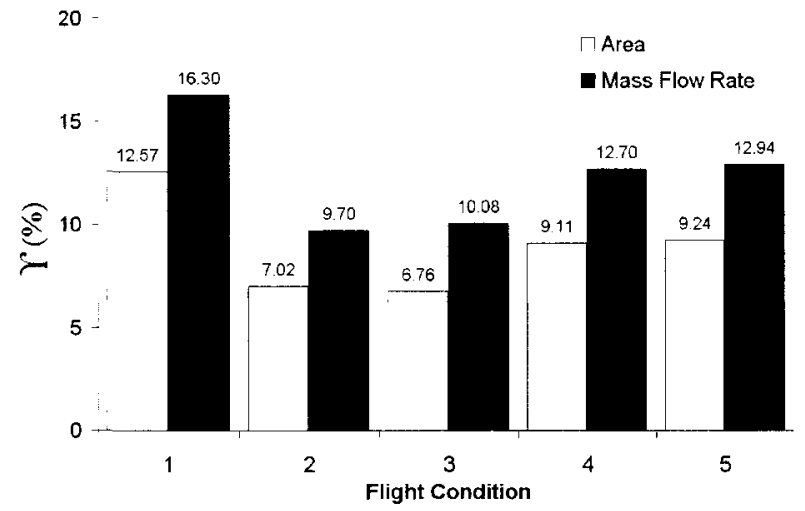

(b) $\Upsilon_{A}$ and $\Upsilon_{m}$ for the selected intake geometry over 5 flight conditions.

Figure 4.3: Flow analysis results for selected geometry over 5 flight conditions.

thesis would have a completely negative affect on an $\mathrm{RBCC}$ vehicles performance, there are several reasons this is not necessarily the case.

First of all, this RBCC air intake geometry is not only required for the rocket-ejector configuration, but is also required to provide a suitable flow path for the other air breathing engine cycles (ramjet, scramjet) used in an RBCC engine. Both the ramjet and scramjet engines would require fuel injectors to operate, which would be used as afterburners during operation in rocket-ejector mode. The afterburners would increase thrust augmentation 


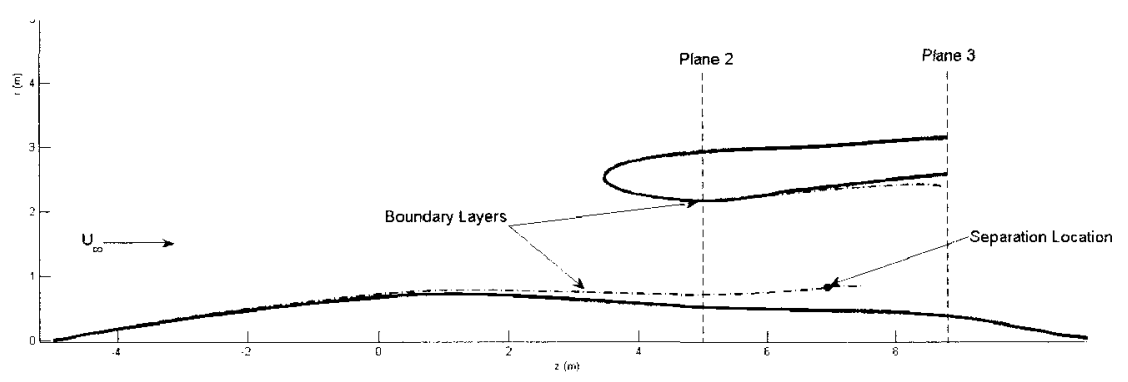

Figure 4.4: Selected air intake geometry along symmetry plane 1 with boundary layer shown for flight condition 4 .

and therefore increase $\Phi$.

Secondly, the flight profile used is intended for use by a conventional rocket powered launch vehicle, which climbs rapidly up to altitude to avoid high atmospheric drag. An air breathing RBCC vehicle would likely spend more time at lower altitudes to take advantage of its air breathing engine cycles. Rocket-ejector analysis using an adjusted flight profile in which the aircraft climbs at a slower rate indicates that the mission averaged $\Phi$ can be increased by adjustments to the flight profile.

Finally, the rocket-ejector and air intake configuration used here which has an adjusted mission averaged thrust augmentation ratio of $\Phi=0.95$ has a value of $\Phi=1.20$ at flight condition 5. Therefore, even without considering the factors described in the previous paragraphs which would certainly increase the mission averaged $\Phi$, this rocket-ejector and air intake configuration does show improved thrust over a pure rocket at some flight conditions. 


\section{Chapter 5}

\section{CFD Simulations}

Three-dimensional computational fluid dynamics (CFD) simulations have been conducted using ANSYS ${ }^{\circledR}$ CFX 11.0. The results from these CFD simulations are used to determine how accurately the flow analysis used in the design method calculates the flow conditions at plane 3 of the air intake (which is the ejector inflow plane). The CFD simulations will also highlight flow situations where 3D flow effects become important. Four simulations, which represent flight conditions 1-4, from table 4.2, have been conducted. Flight condition 1 is a near static flow condition and therefore has the highest $F C$ ratio and peak lip velocity around the cowl. This condition represents the worst case flow situation around the cowl surface. Flight condition 2 and 3 are intermediate flight conditions that are not predicted to contain regions of flow separation. Flight condition 4 is a higher speed flight condition which is predicted to contain regions of flow separation along the centre body surface. This flight condition is used to assess the accuracy of the separation criteria used in the flow analysis.

The following sections describe the methods used to solve the governing equations of the flow, the turbulence model used, boundary conditions, computational grid, convergence, and finally the simulation results. 


\subsection{Discretization Method}

ANSYS ${ }^{\circledR}$ CFX 11.0 solves the 3D, time dependant Navier-Stokes equations (continuity, momentum, energy) over an unstructured 3D mesh. Each 3D mesh element forms a control volume over which the governing equations are evaluated. The integral forms of the governing equations at each control volume are discretized using a finite volume method, which creates a coupled system of equations. The solution variables and fluid properties are stored at the nodes of each mesh element. However, the discretized forms of the governing equations are functions of flow properties at various integration points throughout each control volume. The properties of the flow field at each integration point are calculated using shape factors specific to the mesh element shape [48].

CFX offers several options for discretization of the advection term: a purely first-order upwind scheme, a Specify Blend scheme, and a High Resolution scheme [48]. The Specify Blend scheme allows the user to set a blend factor which affects the schemes accuracy. This scheme is first-order accurate using a blend factor of 0 and is second-order accurate when using a blend factor of 1 . The High Resolution scheme computes a blend factor locally to be as close to a value of 1 , and therefore second-order accurate, as possible. The local blend factor is only reduced near flow discontinuities. The high resolution scheme is used in this work due to its robustness and accuracy.

All simulation results presented here have been run as steady-state simulations.

\subsection{Turbulence Model}

Several two-equation turbulence models are available in CFX 11.0 including the $k-\varepsilon$ model, the $k-\omega$ model, and the SST model. The $k-\varepsilon$ model uses wall functions to estimate the flow property distribution in the viscous sublayer of a turbulent boundary layer. This turbulence model is known to perform poorly in adverse pressure gradients [49] and 
flows with separated boundary layers [48]. The $k-\omega$ turbulence model was developed to improve prediction of boundary layer separation by using an improved near wall treatment. However, the $k-\omega$ is known to be sensitive to the freestream turbulence dissipation rate [50].

Menter's SST turbulence model combines the $k-\varepsilon$ and $k-\omega$ models by using the $k-\omega$ model near the wall and the $k-\varepsilon$ model in all other regions of flow. This blending of two turbulence models combines the advantages of both models. Bardina et al. [51] have shown that the SST model produces the most accurate results, relative to the $k-\varepsilon$ and $k-\omega$ models, in flows with adverse pressure gradients and flow separation. Because the flow through the air intake geometry is expected to experience adverse pressure gradients as well as flow separation under certain conditions, the SST turbulence model is used in this work for all CFD simulations.

CFD simulations have been run using freestream turbulence intensity levels ranging from $1 \%$ to $10 \%$. The variation of the plane 3 flow properties for flight conditions 2 and 3 is less than $0.002 \%$ between turbulence intensity values of $1 \%$ and $10 \%$. Based on these results, a turbulence intensity of $5 \%$ is used for all CFD simulations.

\subsection{Flow Domain and Boundary Conditions}

Due to the symmetry of the air intake geometry, only the region between symmetry plane 1 and symmetry plane 2 is modeled (Fig 2.1). The shape of the computational domain along symmetry plane 1 used for the CFD simulations is illustrated in figure 5.1 and a top view of the domain is shown in figure 5.2. The dimensions of the computational domain, which are shown in figure 5.1, have been determined from domain size trials. The size of the computational domain was increased until the air intake plane 3 properties $\left(\dot{m}, p_{3}\right.$, etc.) remained constant. Since at some flow conditions flow separation is expected, the 
duct outlet boundary has been placed downstream of plane 3 to avoid having regions of separated flow across this boundary.

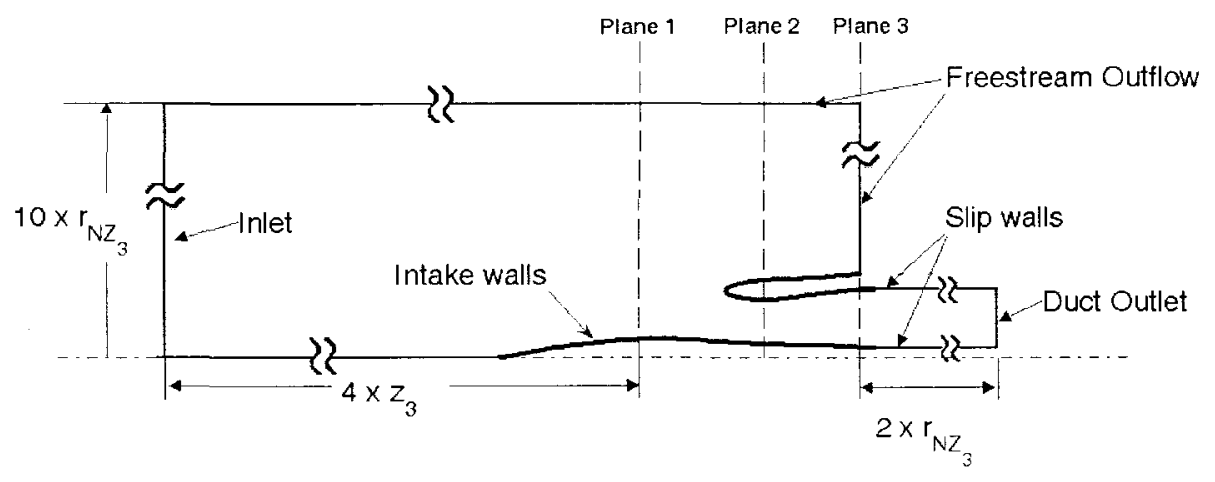

Figure 5.1: View of computational domain along symmetry plane 1 used in CFD simulations with labeled boundary conditions.

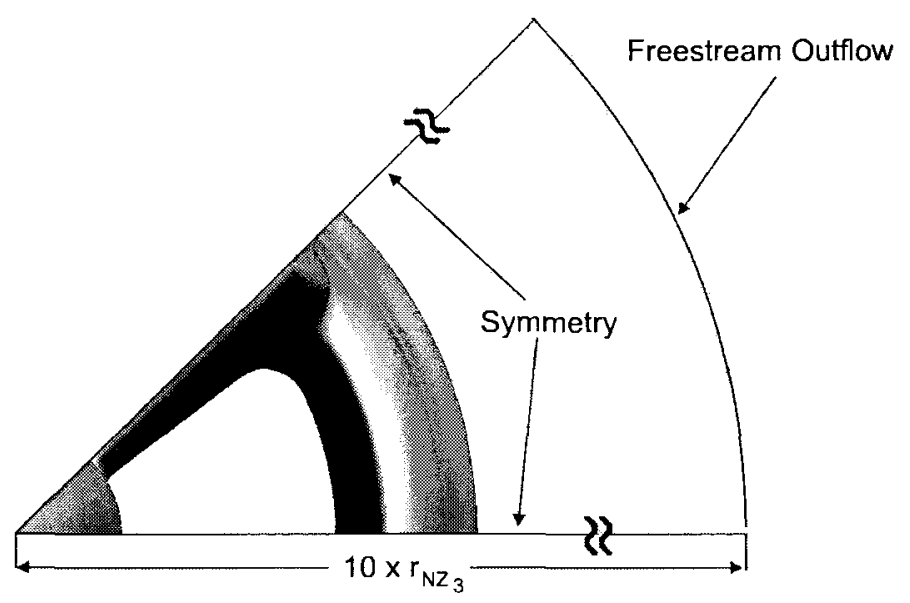

Figure 5.2: Top view of computational domain used in CFD simulations with labeled boundary conditions.

For each CFD simulation air is modeled as an ideal gas, the reference pressure and temperature is set to the freestream static conditions, and the total energy model is used. The air intake walls are specified as no-slip adiabatic walls which is consistent with the wall boundaries used by Reddy and Sree [52] in their RBCC inlet simulations, which used the NPARC Navier-Stokes code. The domain walls downstream of plane 3 are defined 
Table 5.1: CFD boundary conditions.

\begin{tabular}{c|c|cccc|} 
& & \multicolumn{4}{|c}{ Flight Condition } \\
Boundary & Parameter & 1 & 2 & 3 & 4 \\
\hline \multirow{2}{*}{ Inlet } & Velocity $\left(\mathrm{ms}^{-1}\right)$ & 3.7 & 64.0 & 136.0 & 208.3 \\
& Temperature $(\mathrm{K})$ & 288 & 282.2 & 273.9 & 263.5 \\
& Pressure $(\mathrm{kPa})$ & 100.9 & 90.7 & 77.7 & 63.4 \\
\hline Duct Outlet & Pressure $(\mathrm{kPa})$ & 98.1 & 89.4 & 83.3 & 79.6 \\
\hline Outflows & Pressure $(\mathrm{kPa})$ & 100.9 & 90.7 & 77.7 & 63.4 \\
& Temperature $(\mathrm{K})$ & 288 & 282.2 & 273.9 & 263.5 \\
\hline
\end{tabular}

as slip walls. Slip walls are used in this region to prevent the boundary layer that would otherwise develop downstream of plane 3 from significantly affecting the flow field. The domain inlet, shown in figure 5.1 has been defined as a velocity inlet with a uniform velocity acting normal to the inlet surface. The magnitude of the inlet velocity is equal to $U_{\infty}$ for each flight condition. Based on the turbulence intensity results from section 5.2, a turbulence intensity of $5 \%$ has been specified. The freestream outflow planes, shown in figure 5.1 and figure 5.2, are specified as pressure openings with an average pressure equal to the reference pressure. Symmetry plane 1 and 2 are defined as symmetry boundaries, shown in figure 5.2. Finally, the duct outlet is specified as a pressure outlet with an average static pressure condition. Since the duct outflow plane is set downstream of plane 3 , the average static pressure at plane 3 cannot be set directly. Therefore, the value of the average static pressure for each flight condition is adjusted until the final steady-state mass flow averaged static pressure at plane 3 is equal to $p_{3}$ for the desired flight condition. The properties of the boundary conditions for the CFD simulations are presented in table 5.1. 


\subsection{Grid and Grid Convergence Study}

Unstructured tetrahedral meshes were built using ANSYS ${ }^{\circledR}$ ICEM 11.0. Figure 5.3 and figure 5.4 show an example of the mesh along symmetry plane 1 and symmetry plane 2 respectively. The 3D mesh is defined by specifying the maximum and minimum cell size along each intake surface. The mesh density is increased in regions where large velocity gradients are expected, such as around the centre body nose, the cowl lip, and the fairing leading edge. The tetrahedral mesher ANSYS ${ }^{\circledR}$ ICEM 11.0 then fills the computational domain using the surface mesh and mesh density information. Prism layers are then added along the intake surfaces in order to resolve the boundary layers. Because the SST turbulence model is a low-Reynolds model the height of the first node off the wall is specified such that $y^{+} \leq 2$ [48]. The boundary layer results from the intake design method results are then used to determine the total height of the prism layer along the intake surfaces for a given flight condition. The prism layers are specified such that a minimum of 12 nodes are within the boundary layer.

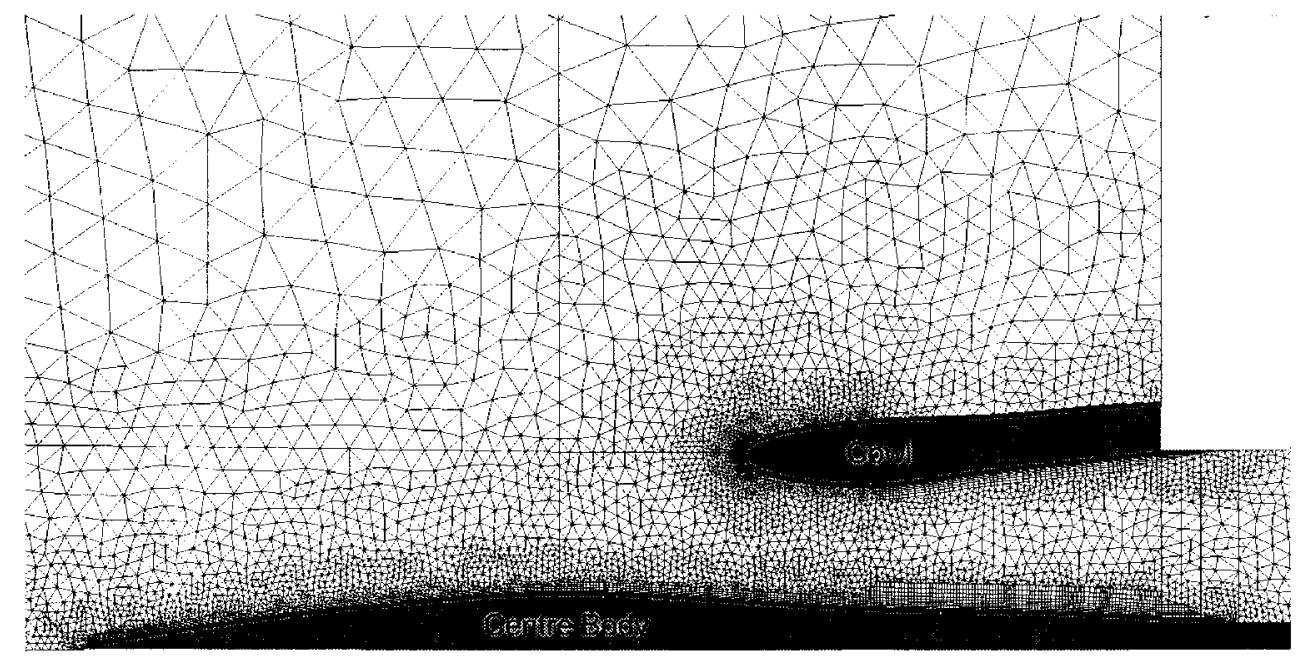

Figure 5.3: View of computational mesh along symmetry plane 1 .

A grid convergence study has been conducted using a coarse, medium and fine grid of the same intake geometry operating at flight condition 3 . The number of elements in each 


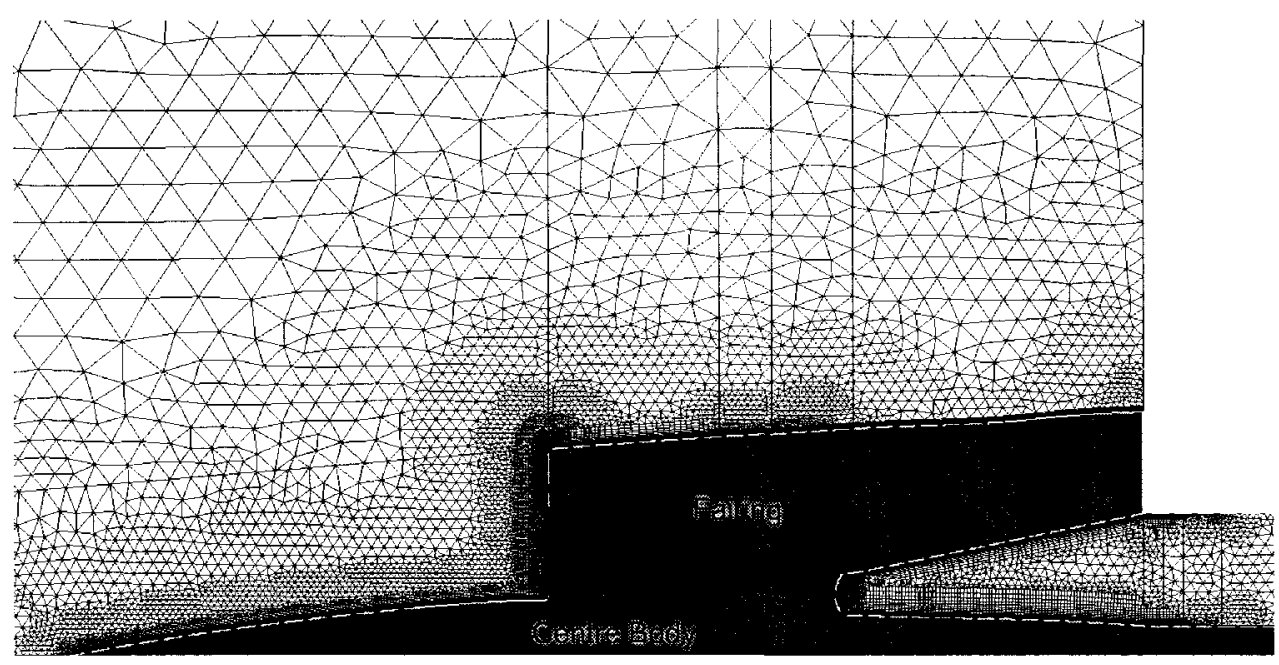

Figure 5.4: View of computational mesh along symmetry plane 2.

Table 5.2: Grid sizes

\begin{tabular}{c|c} 
Grid & Elements \\
\hline Fine & 6607184 \\
Medium & 2840800 \\
Coarse & 1194397
\end{tabular}

grid is listed in table 5.2 .

The different grids have been systematically generated by multiplying the mesh cell sizes and densities by a refinement factor. Freitas et al [53] recommends an overall grid refinement factor (GRF) of 1.3 or greater, where GRF is defined as

$$
G R F=\frac{\Omega_{\text {medium }}}{\Omega_{\text {fine }}}=\frac{\Omega_{\text {coarse }}}{\Omega_{\text {medium }}}
$$

where $\Omega$ is a representative cell length and is defined as

$$
\Omega=\bar{v}^{\frac{1}{3}}
$$


where $\bar{\mho}$ is the average grid cell volume for a given grid. The final GRF between the fine and medium grids is 1.32 and the GRF between the medium and coarse grids is 1.33 . A plot of the average Mach number at a given axial location through the duct region of the air intake is plotted in figure 5.5 for all three grids. The results show no significant variation between the fine and medium grids while the coarse grid results under predict the Mach number as compared to the medium and fine grid results by at most $1 \%$.

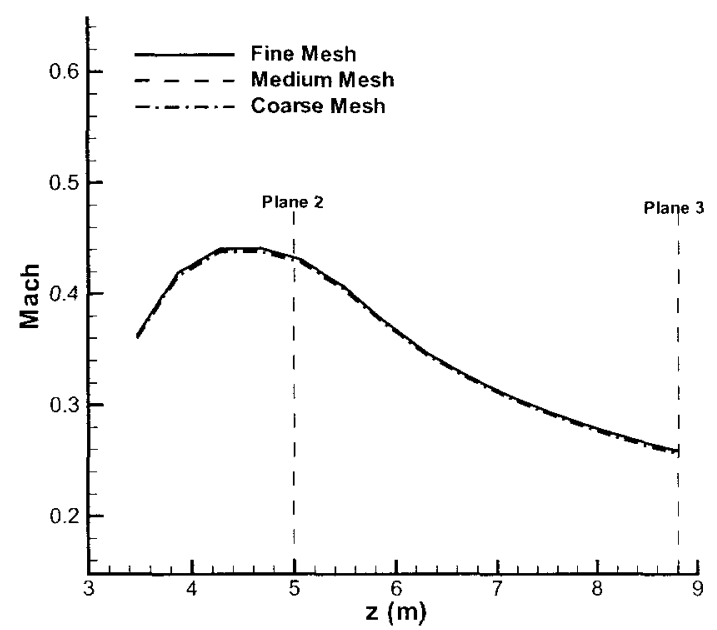

Figure 5.5: Mean Mach number distribution for three grids.

The numerical uncertainty associated with the grid used in the air intake CFD simulations can be estimated by the grid convergence index (GCI). Calculation of the GCI between the medium and fine grids has been carried out using the method described by Freitas et al [53]. Results from this analysis are summarized in table 5.3.

The grid convergence results show that there is very little variation between the medium and fine grids, with $G C I_{\frac{\text { medium }}{\text { fine }}}$ less than $1 \%$ for several parameters of interest. For example, the Mach number at plane 3 can be reported with a confidence interval of $95 \%$ [53] to be $M_{3}=0.254 \pm 0.00143(0.563 \%)$. Therefore, all CFD simulations in this work have been conducted on grids equivalent to or finer than the medium grid size $(\geq 3$ 
Table 5.3: Grid convergence study results

\begin{tabular}{c|cc} 
Flow Parameter & Value & $G C I_{\frac{\text { medium }}{\text { fine }}}(\%)$ \\
\hline$M_{3}$ & 0.254 & 0.563 \\
$A_{3}^{(\text {visc })}\left(\mathrm{m}^{2}\right)$ & 18.171 & 0.208 \\
$\dot{m}(\mathrm{~kg} / \mathrm{s})$ & 1604.82 & 0.299
\end{tabular}

million elements).

\subsection{Convergence History}

The mass and momentum residual history for the flight condition 3 simulation is shown in figure 5.6. Residuals are a measure of the imbalance in the control volume equations. Residuals for each control volume equation are normalized with respect to a representative range for the entire flow domain [41]. The root-mean-squared (RMS) residuals are plotted in figure 5.6. The convergence history results show that the mass and $\mathrm{z}$-momentum

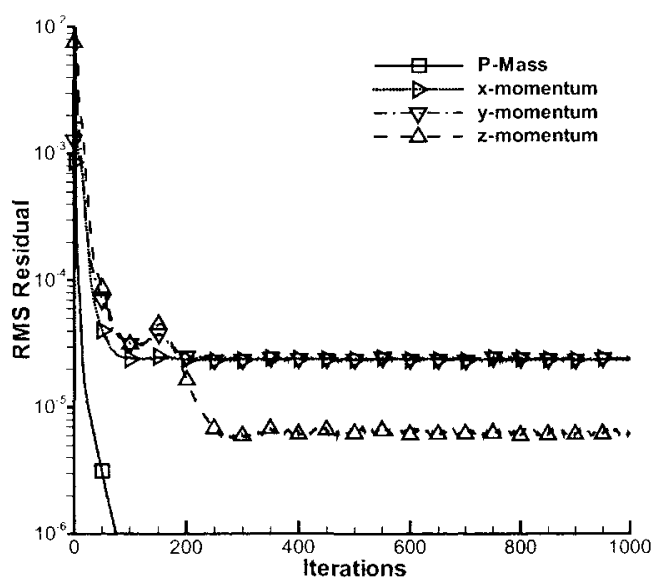

Figure 5.6: RMS residuals history for the flight condition 3 simulation. 
equation RMS-residuals drop below 1E-5 within 200 iterations and all equation RMSresiduals level off after approximately 300 iterations. RMS-residual values below 1E-5 are considered sufficient for engineering applications [48], with the maximum residual values one order of magnitude higher than RMS values [41]. The maximum values of the mass and z-momentum residuals met this criteria, however; the maximum values of the $\mathrm{x}$-momentum and $\mathrm{y}$-momentum residuals are more than 3 orders of magnitude greater than the RMS values. The high maximum $x$ and $y$ momentum residuals are an indication of local regions of high equation residuals in the flow domain. Examination of the flow domain shows that mesh volumes with $\mathrm{x}$ and $\mathrm{y}$ momentum residual values above $1 \mathrm{E}-5$ are clustered at the fairing leading edge close to the centre body, shown in figure 5.7, where there are large velocity gradients. While the total volume of these high residual locations is small relative to the total domain volume, the large residual values of this volume skews the RMS-residuals. Reduction of the mesh volume size in these regions has been found to reduce maximum residual values, however; meshing errors and memory constraints have limited the degree of refinement to date.

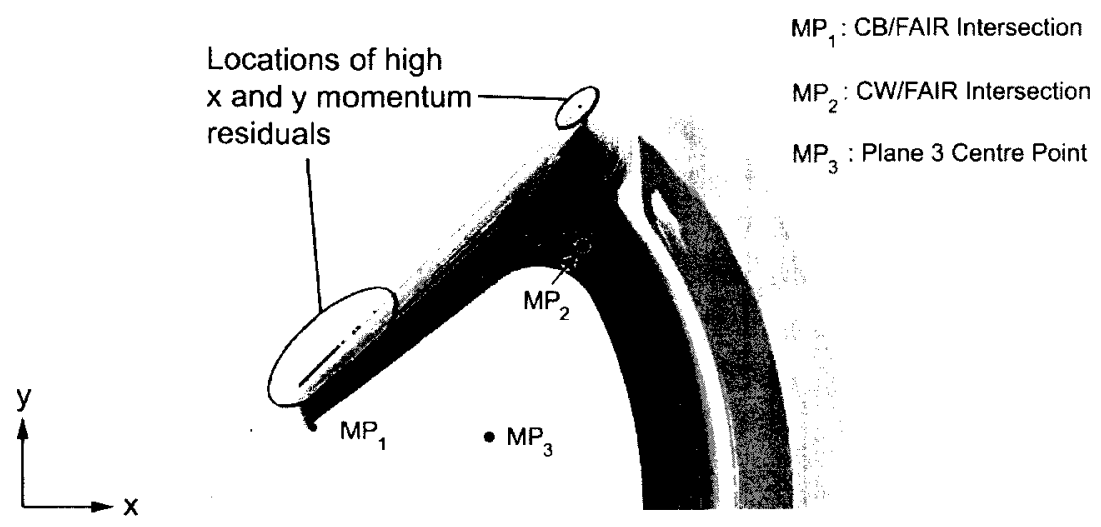

Figure 5.7: Top view of CFD flight condition 3 simulation results with locations of high residuals highlighted. Static pressure contours are shown on air intake surfaces. Locations of monitor points are also indicated.

Residual history results for the other flight conditions show similar behaviour to those shown in figure 5.6. The $\mathrm{x}$ and $\mathrm{y}$ momentum RMS-residuals for flight condition 1, where 


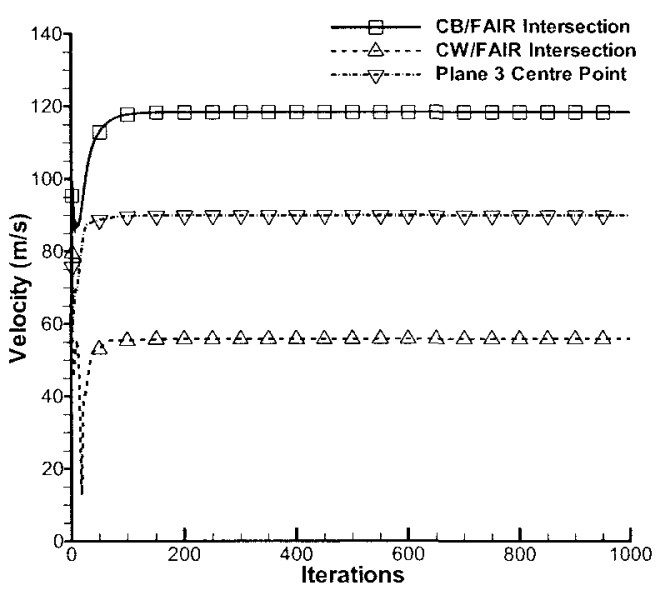

Figure 5.8: Monitor point history for flight condition 3 simulation.

the freestream velocity is low, settle out at values of approximately $1 \mathrm{E}-5$ and thus do not contain the local regions of high residuals as seen at other flight conditions. The velocity at three monitor points, shown in figure 5.7 throughout the domain is plotted against iteration number in figure 5.8 for flight condition 3. For flight condition 3 , the maximum difference in velocity for all three points once the RMS residuals stabilize is $0.021 \%$. The largest difference in velocity at the monitor points for the CFD simulations for flight conditions $2-4$ is $0.09 \%$.

\subsection{CFD Results}

Comparisons between the air intake design and the 3D CFD simulations is divided into 4 sections: pressure distribution, boundary layer, total pressure loss, and mass flow rate. Each section will discuss the validity of the methods and simplifying assumptions made in the air intake design method flow analysis. Pressure distribution comparisons will be used to evaluate the accuracy of the flow analysis panel method, velocity scaling and compressibility correction. Boundary layer comparisons will be used to illustrate the accuracy 
of the compressible integral boundary layer method used. Total pressure loss comparisons will be used evaluate the accuracy of the total pressure loss calculations, and thus the cal-

culations of $\rho_{3}^{(v i s c)}$ and $U_{3}^{(v i s c)}$. Finally, the overall accuracy of the air intake design method flow analysis will be evaluated with air intake mass flow rate comparisons.

\subsubsection{Pressure Distribution}

The static pressure distributions along symmetry plane 1 of the centre body and cowl surfaces have been plotted in figure 5.9 for flight condition 1 and flight condition 3 . The results show that while the shape of the pressure distribution calculated by the design method is similar to the CFD results, the design method tends to predict a lower minimum pressure than the CFD results. For flight condition 1, the design method under predicts the static pressure at plane 2 by about $2 \%$ on the centre body and by about $1.8 \%$ on the cowl. For flight condition 3 , the design method under predicts the static pressure at plane 2 by about $1 \%$ along the centre body and by $2 \%$ along the cowl surface. This under prediction of the static pressure magnitude may be a result of the invisicid flow solver not taking into account flow area blockage due to boundary layer development as well as losses in total pressure due to friction.

As discussed in section 3.2.3, the velocity scaling method is used to account for the presence of the fairing geometry. The importance of accounting for the fairing geometry is seen most clearly in figure 5.9(c) and (d), where the pressure distribution calculated using the incompressible, unscaled velocity distribution is also plotted. In figure 5.9(c), the unscaled pressure distribution lacks the two troughs found in the final design method and CFD results. The first trough downstream of plane 1 is centred around the location where the rocket nozzle intersects the centre body. At this intersection point, the fairing geometry is at its maximum thickness and there is a velocity peak in this region, which causes the drop in static pressure. The second trough downstream of plane 1, which corresponds 


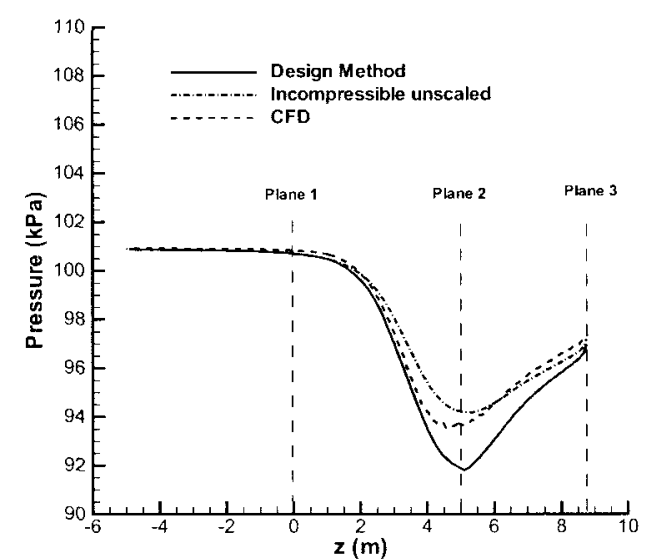

(a) Centre body at flight condition 1 .

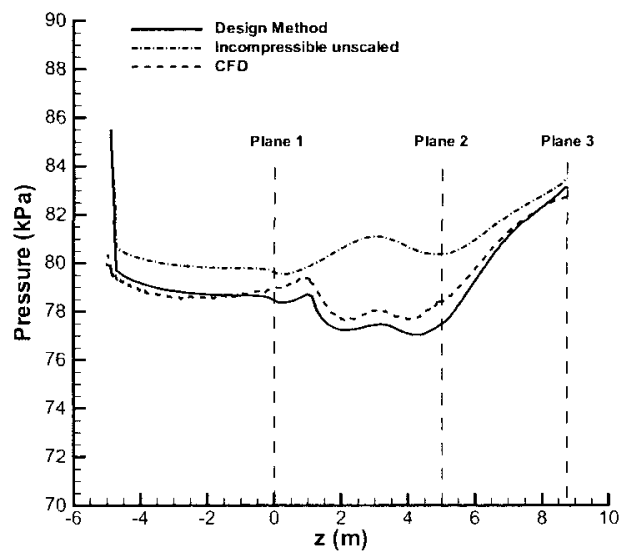

(c) Centre body at flight condition 3 .

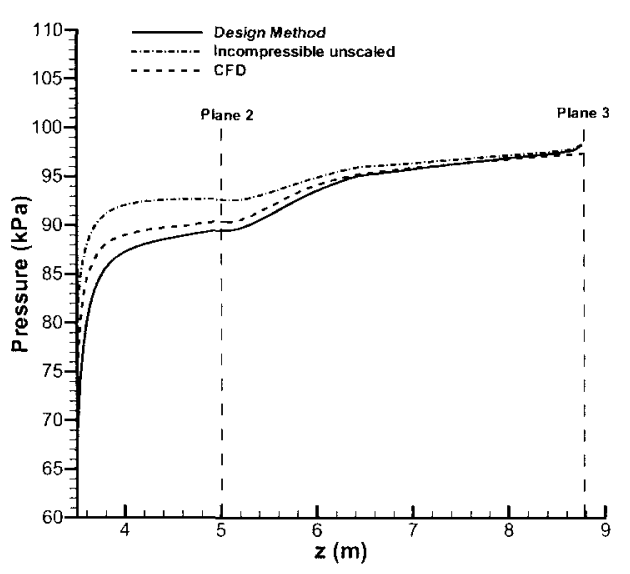

(b) Cowl at flight condition 1.

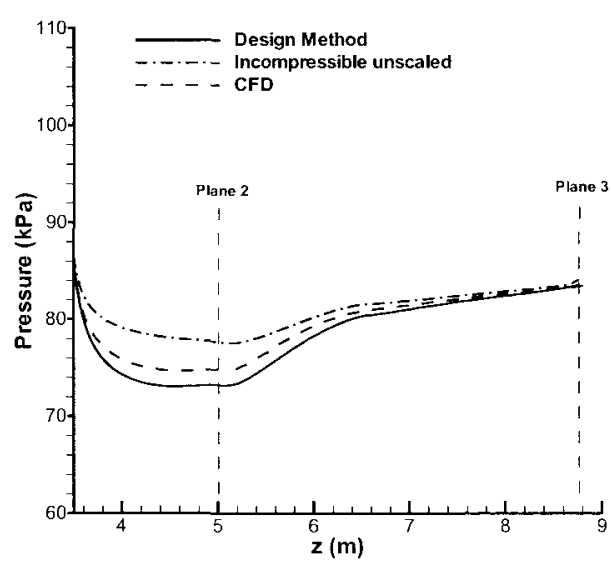

(d) Cowl at flight condition 3.

Figure 5.9: Comparison of design method and CFD static pressure distributions along symmetry plane 1 .

to the location of the cowl lip, is caused due to a reduction in flow area due to the fillet surface between the cowl lip and fairing surfaces. In the absence of any velocity scaling, these pressure troughs are not captured. There is also a subtle change in the pressure distribution along the cowl surface, shown in figure 5.9(d), which is again related to the altered area distribution around the cowl lip due to the fillet surface. 


\subsubsection{Boundary Layer}

Comparisons of the boundary layer height $\delta$ on the centre body and cowl surfaces along symmetry plane 1 calculated by the design method and the CFD simulations are presented in figure 5.10. The design method prediction of the boundary layer along the cowl surfaces is shown in figure 5.10(b) and (d). At flight condition 1, which operates at a flow capture ratio of 32 , the design method predicts a thicker cowl boundary layer and flow separation along the cowl due to the larger adverse pressure gradient, shown in figure 5.9(b), calculated by the design method. The boundary layer height calculated by the design method over predicts $\delta$ at the separation point by $32 \%$ for flight condition 1 . At flight condition 3 , the design method over predicts $\delta$ at plane 3 by $1 \%$. Overall the method tends to over predict $\delta$ along the cowl surface due to larger predicted adverse pressure gradients.

Along the centre body, the boundary layer height calculated by the design method tends to be thicker, shown in figure $5.10(\mathrm{a})$ and (c), at both flight conditions. For flight condition 1 the design method predicts a $\delta$ at plane 3 which is $37 \%$ greater than the CFD result and for flight condition $3, \delta$ is over predicted by $16 \%$. Overall, for both the cowl and centre body surfaces, the magnitude of the error in $\delta$ decreases as flight speed increases.

The larger adverse pressure gradient predicted by the design method (Fig 5.9(a),(c)), as well as differences in the pressure distribution upstream of plane 2 contributes to the thicker boundary layer predictions along the centre body. A comparison of the design method and CFD boundary layer height along the entire centre body surface for flight condition 3 is shown in figure 5.11. These results show that the boundary layer height predicted by the design method matches well with the CFD results up to about $z=3 m$, which corresponds to the axial location just upstream of the cowl lip. Downstream of this location, the design method boundary layer thickens relative to the CFD boundary layer. The thicker design method boundary layer downstream of the cowl lip is likely caused by the larger adverse pressure gradient, shown in figure $5.9(\mathrm{c})$, predicted by the design 


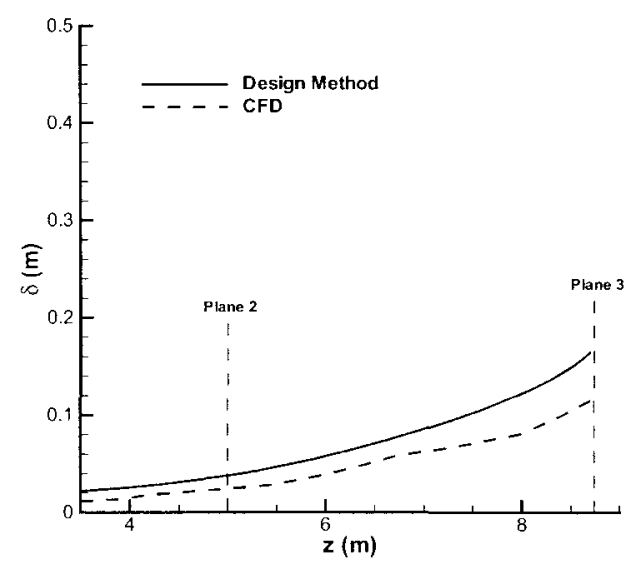

(a) Centre body at flight condition 1 .

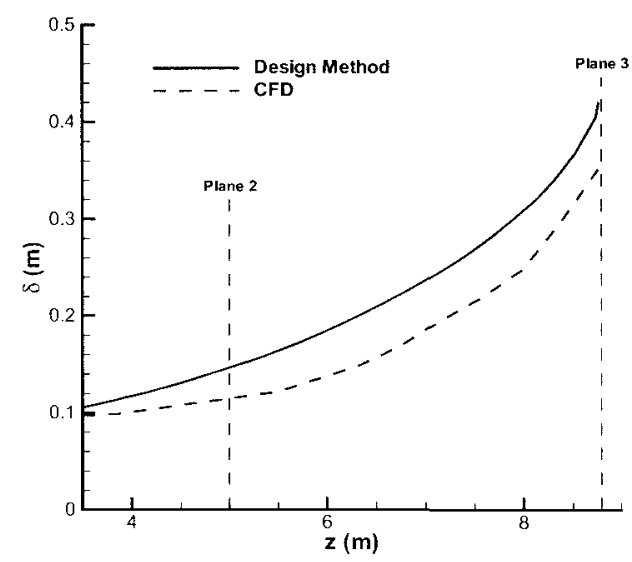

(c) Centrc body at flight condition 3 .

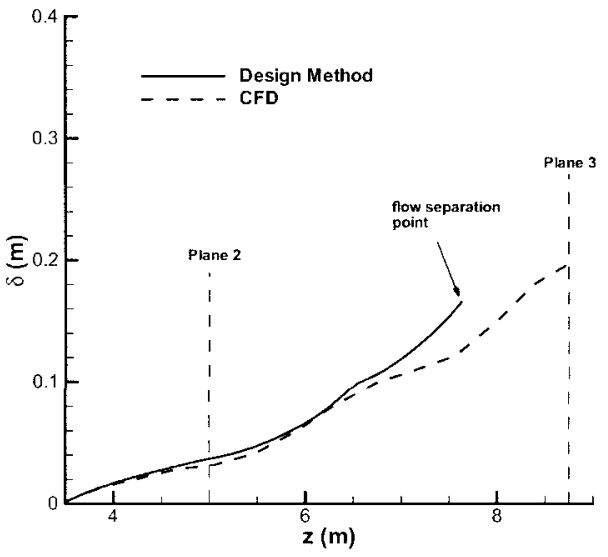

(b) Cowl at flight condition 1 .

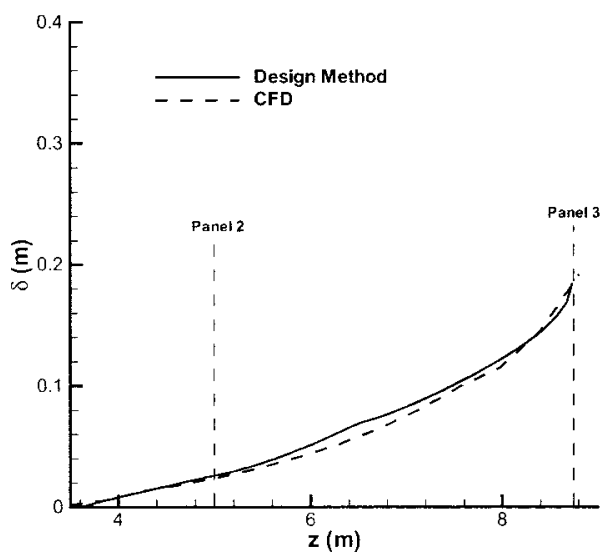

(d) Cowl at flight condition 3 .

Figure 5.10: Comparison of design method and CFD $\delta$ on the intake surfaces along symmetry plane 1 .

method.

The discrepancy in the pressure distribution shape upstream of plane 2 can be attributed to the flow stagnating along the fairing leading edge in the CFD simulations, as well as to the velocity scaling calculations used upstream of plane 2. However, figure 5.11 shows that the primary cause of the thicker design method boundary layer is the larger design 


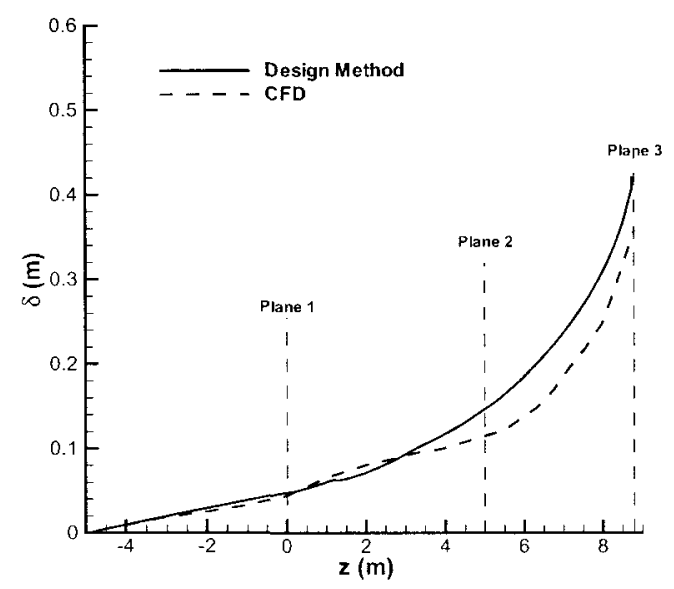

Figure 5.11: Boundary layer thickness along centre body at flight condition 3 .

method adverse pressure gradient.

Figure 5.12 shows a velocity contour plot along symmetry plane 1 of the intake operating at flight condition 4 where separation is expected. The air intake design method predicts a separation location along symmetry plane $112 \%$ of $z_{3}$ downstream of the CFD separation location. Examination of the CFD results show that the boundary layer does not separate across the entire centre body surface at the separation location along symmetry plane 1 . This result indicates that $3 \mathrm{D}$ effects, which cannot be predicted by the design method, are contributing to the discrepancy between the design method and CFD results. In addition, comparisons made by Childs [20] between experimental results on a planar diffuser and the strongly interacting Childs boundary layer method, where the calculated pressure distribution matched well with experimental results, show that the Childs boundary layer method tends to predict flow separation downstream of the experimental separation location.

The behaviour of the separated flow region downstream of the separation point also differs between the CFD and design method. In the CFD simulations, after boundary layer separation, the boundary of the separated flow region initially expands into the core flow 
and then extends downstream with a reduced expansion rate. In the design method, just prior to boundary layer separation the boundary layer height expands quickly and then is assumed to extend straight downstream from the separation point as shown in figure 4.4.

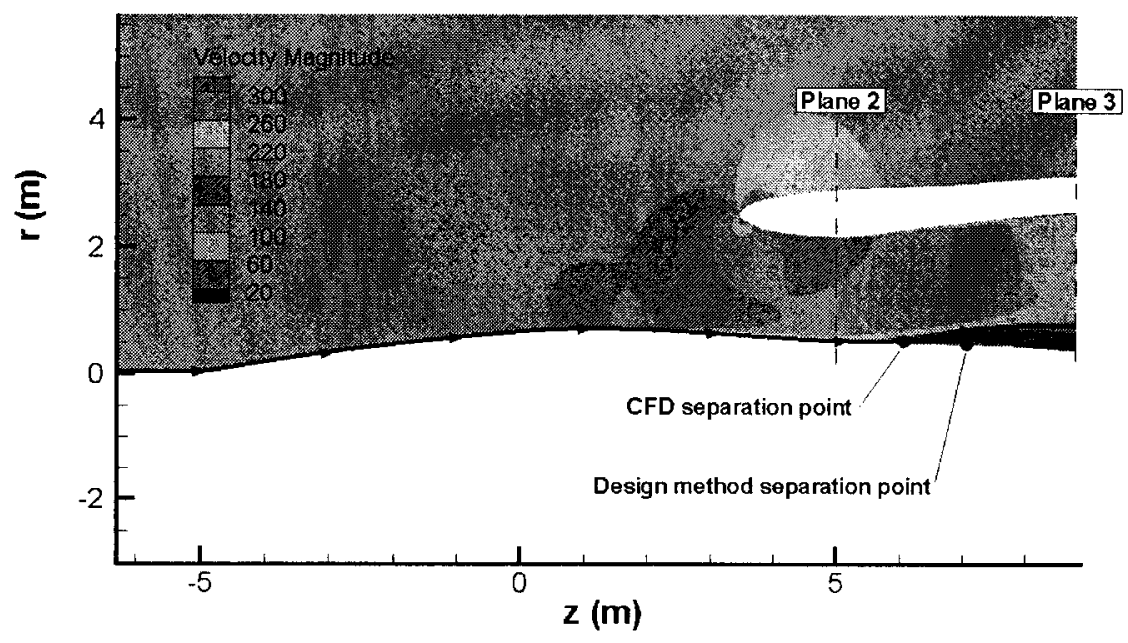

Figure 5.12: CFD velocity contour with CFD and design method boundary layer separation locations for flight condition 4.

A flow feature that is present in the CFD simulation for flight condition 1 and that cannot be predicted by the current design method is shown in figure 5.13(a)-(b). A small region of separated flow forms on the lip side of the lip/fairing fillet, at high flow capture ratios (i.e. low freestream velocities), and continues to grow downstream to plane 3. Figure 5.13(a) shows an $x-y$ velocity contour at plane 3 with the region of separated flow highlighted. Figure 5.13(b) shows intake surface pressure contours and surface streamlines along the intake lip and fairing geometry. At large flow capture ratios, the incoming flow must wrap around the lip surface and the lip/fairing fillet surfaces. There is a large velocity peak at the leading edge of the lip/fairing fillet surface, identified in figure 5.13(b), which causes the streamlines passing over this point to experience a larger adverse pressure gradient than the streamlines flowing over the lip surface alone. Consequently, the boundary layer thickens in the region downstream of this velocity peak and eventually forms a region of separated flow. This region of flow separation does not appear to be associated with the 
regions of high residuals (Fig 5.7) since the high residual regions are concentrated along the leading edge of the fairing geometry.

This region of separated flow at flight condition 1 is distinct from the region of separation observed at flight condition 4 along the centre body, which can be predicted by the design method. The current air intake design method cannot predict the formation of the separated flow region at flight condition 1 because it is a highly $3 \mathrm{D}$ flow feature, however; this type of flow behaviour has only been observed at flight condition 1 where the flow capture ratio is relatively large.

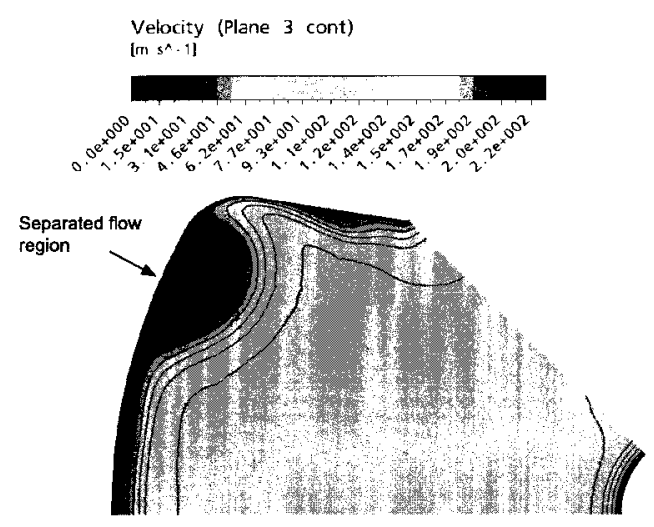

(a) $\mathrm{x}-\mathrm{y}$ velocity contour of plane 3 .

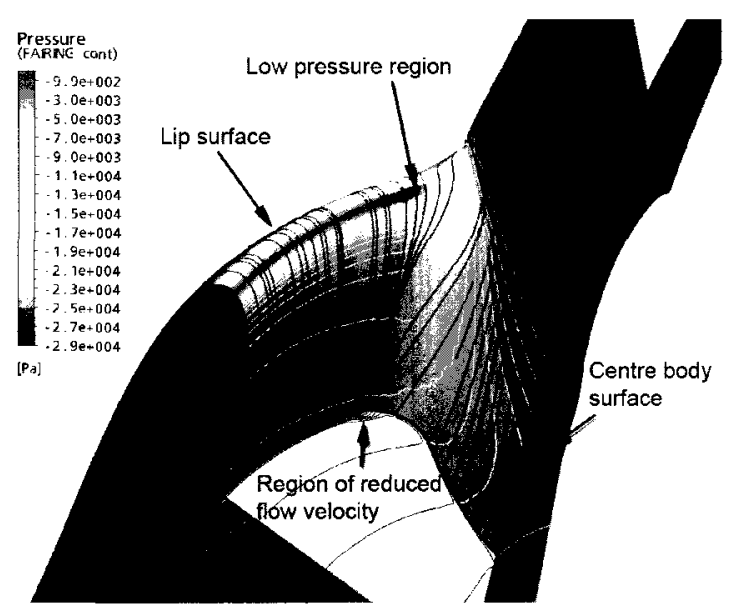

(b) View of static pressure contours and surface streamlines.

Figure 5.13: Flow condition 1 flow feature around cowl lip.

The boundary layer data directly affects the effective flow area at plane $3, A_{3}^{(v i s c)}$, which in turn affects the design method calculation of $\dot{m}$. The percent difference of $A_{3}^{(v i s c)}$ calculated by the design method relative to the CFD simulations is shown in figure 5.14. At flight conditions $1-4$, the design method under predicts $A_{3}^{(v i s c)}$ by about $3.0 \%-8.8 \%$ due to the over prediction of the boundary layer thickness along the centre body and cowl surfaces. 


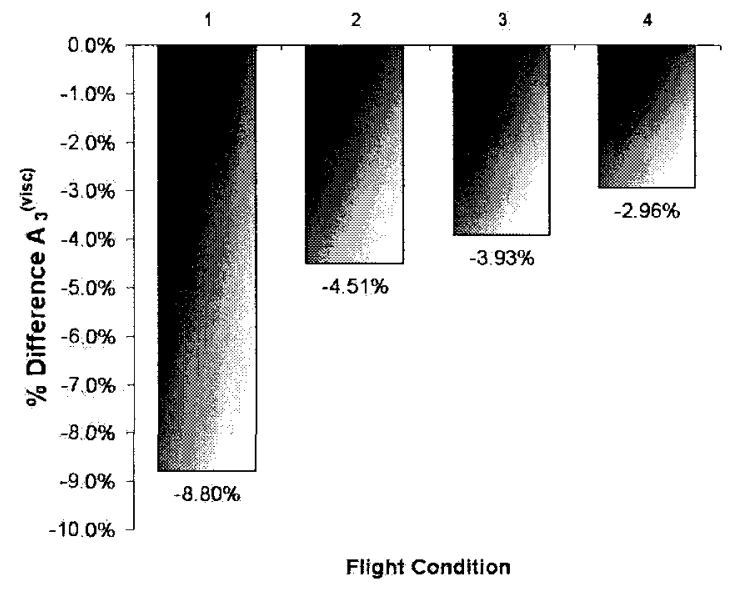

Figure 5.14: Percent difference of design method $A_{3}^{(v i s c)}$ from CFD $A_{3}^{(v i s c)}$ for different flight conditions.

The trend in figure 5.14 shows that as flight speed increases, the error in $A_{3}^{(v i s c)}$ decreases. This trend is consistent with the trends in the boundary layer height error along the centre body and cowl between flight condition 1 and 3 . The error in $A_{3}^{(\text {visc })}$ at flight condition 1 is the largest relative to the other flight conditions because separation is predicted along the cowl surface whereas the CFD results do not show significant flow separation. Flow separation along the cowl surface tends to have a more significant effect on the calculated $A_{3}^{(v i s c)}$ since the radius of the cowl surface is typically large compared to the centre body surface and thus errors in $\delta^{*}$ along the cowl are more significant than errors in $\delta^{*}$ along the centre body.

At flight condition 4, the design method predicts boundary layer separation along the centre body downstream of the CFD simulation results, which would tend to cause an over prediction of $A_{3}^{(v i s c)}$ by the design method. However due to differences in the boundary layer development between the centre body along symmetry plane 1 and the intersection between the centre body and fairing surfaces, the boundary layer in the CFD simulations does not separate across the entire centre body surface, which results in reduced flow 
blockage due to boundary layer separation. The differences in the boundary layer development along the sides of the centre body surface are caused by the fairing surface altering the pressure distribution along the centre body and fairing surface intersection, as well as due to the interaction of the fairing boundary layer and the centre body boundary layer. These $3 \mathrm{D}$ flow effects can not be predicted by the design method. Consequently, the design method still under predicts $A_{3}^{(v i s c)}$ by $2.96 \%$.

\subsubsection{Total Pressure Loss}

In addition to evaluating the accuracy of the design methods calculation of $A_{3}^{(v i s c)}$, the loss in total pressure at plane 3 of the air intake is predicted in order to estimate the values of $\rho_{3}^{(v i s c)}$ and $U_{z_{3}}^{(v i s c)}$ according to the equations in section 3.4. The values of $\rho_{3}^{(v i s c)}$ and $U_{z_{3}}^{(v i s c)}$ are then used along with $A_{3}^{(v i s c)}$ to calculate the intake mass flow rate. Comparisons between the design method and CFD total pressure loss distribution through the air intake are shown in figure 5.15 .

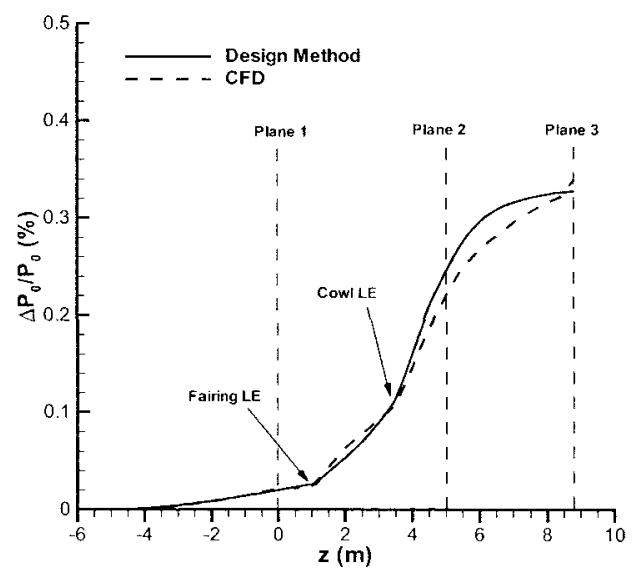

(a) Flight condition 3 .

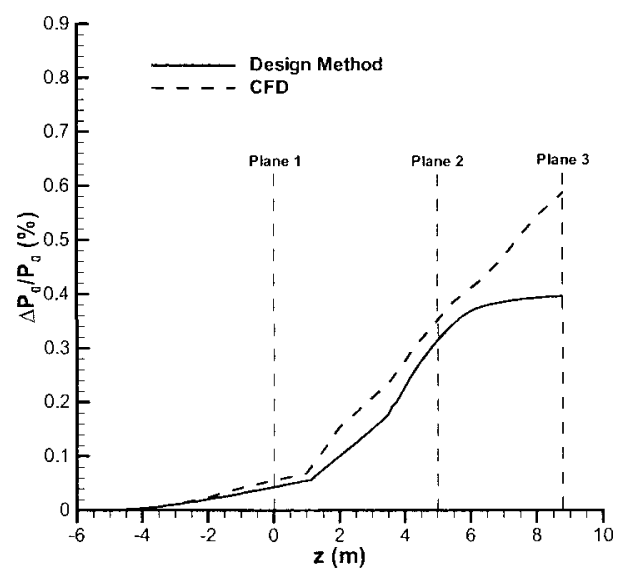

(b) Flight condition 4 .

Figure 5.15: Total pressure loss distrubutions.

At flight condition 3 , the shape of the design method $\Delta P_{0} / P_{0}$ distribution, shown in 
figure 5.15(a) matches the CFD results reasonably well. The two changes in the $\Delta P_{0} / P_{0}$ distribution slope between plane 1 and plane 2 show where the fairing and cowl surfaces begin to contribute to total pressure losses. Both the design method and CFD $\Delta P_{0} / P_{0}$ distributions display these changes in $\Delta P_{0} / P_{0}$ distribution slope. The shape of the design method $\Delta P_{0} / P_{0}$ distribution for flight condition 4 , where the freestream Mach number is 0.64 , shown in figure 5.15 (b) matches the CFD results reasonably well up to the beginning of the fairing. Downstream of the fairing the design method tends to under predict $\Delta P_{0} / P_{0}$. This under prediction of $\Delta P_{0} / P_{0}$ upstream of plane 2 may be a result of the design method not accounting for 3D flow affects as well as the assumption that total pressure loss in a compressible flow is directly related to friction force as described in section 3.3.2. The $\Delta P_{0} / P_{0}$ distribution for the CFD results continues to increase downstream of $z=6.0 \mathrm{~m}$, which is the location of boundary layer separation in the CFD results, whereas the $\Delta P_{0} / P_{0}$ distribution calculated by the design method begins to level off. The discrepancy between the design method and CFD $\Delta P_{0} / P_{0}$ distributions is due to the design method $\Delta P_{0}$ calculations not being valid for separated flow. Since the $\Delta P_{0}$ calculations require local skin friction coefficient, $C_{f}$ data, and the boundary layer calculations are terminated once separation is detected, values of $C_{f}$ are not available in separated flow regions. Past the separation point, the design method simply extrapolates the $\Delta P_{0} / P_{0}$ distribution to plane 3 using the slope of the $\Delta P_{0} / P_{0}$ distribution at the separation point.

The percent difference of $P_{0_{3}}^{(\text {visc })}$ calculated by the design method relative to the CFD simulations is shown in figure 5.16 .

At flight condition 1 the total pressure loss is over predicted, and thus $P_{0_{3}}^{(\text {visc })}$ is under predicted, due to the design method predicting flow separation along the cowl surface while the CFD results do not show significant flow separation along the cowl. In addition, the design method predicts a flow stagnation point further downstream along the cowl outer surface than the CFD results. From flight condition $2-4$, the total pressure loss is under predicted and thus $P_{0_{3}}^{(\text {visc })}$ is over predicted. Overall, the error in $P_{0_{3}}^{(\text {visc })}$ from the CFD 


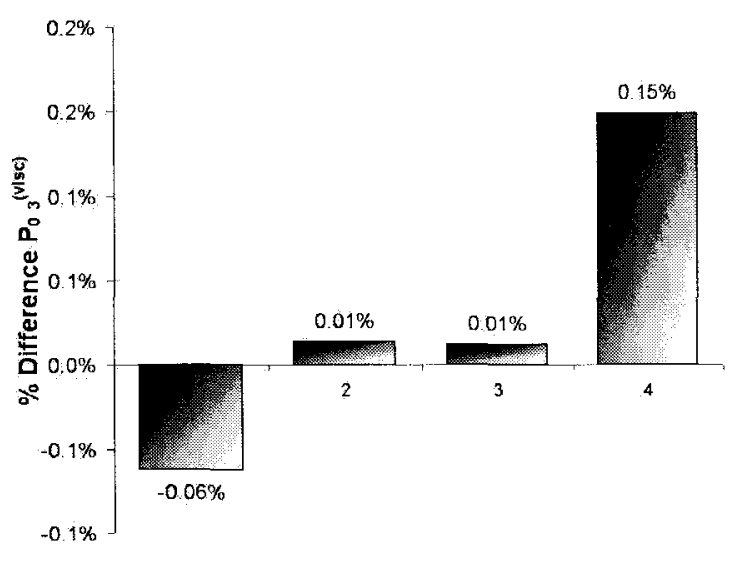

Flight Condition

Figure 5.16: Percent difference of design method $P_{0_{3}}^{(\text {visc })}$ from CFD $P_{0_{3}}$ for different flight conditions.

simulations is quite low for all flight conditions tested, which is in part due to the low total pressure loss relative to total pressure at plane 3 .

As discussed previously, the $\Delta P_{0}$ results are used to calculate $\rho_{3}^{(v i s c)}$ and $U_{z_{3}}^{(v i s c)}$, which are then used to calculate $\dot{m}$. Figure 5.17 shows how $\alpha$, the intakc air mass flow rate over rocket exhaust mass flow rate, from the CFD results compares to the design method $\alpha$ with and without the total pressure loss corrections.

The results in figure 5.17 show that without considering total pressure losses, where $\dot{m}$ is calculated using the corrected flow area along with the uncorrected density and velocity, the calculated $\alpha$ is under predicted from flight conditions $1-3$, but is over predicted at flight condition 4 . When adjustments are made to $\rho_{3}^{(v i s c)}$ and $U_{z 3}^{(\text {visc })}$ due to estimating total pressure losses, the calculated $\alpha$ is shifted down such that $\alpha$ is under predicted for all flight conditions tested. In addition, when total pressure losses are accounted for, $\alpha$ decreases between flight condition 2 and 3 which is consistent with the CFD results, whereas $\alpha$ increases between flight condition 2 and 3 when total pressure losses are not considered. The total pressure corrections used in this design method therefore tend to increase the under prediction of $\alpha$ and thus predict more conservative values of $\dot{m}$. 


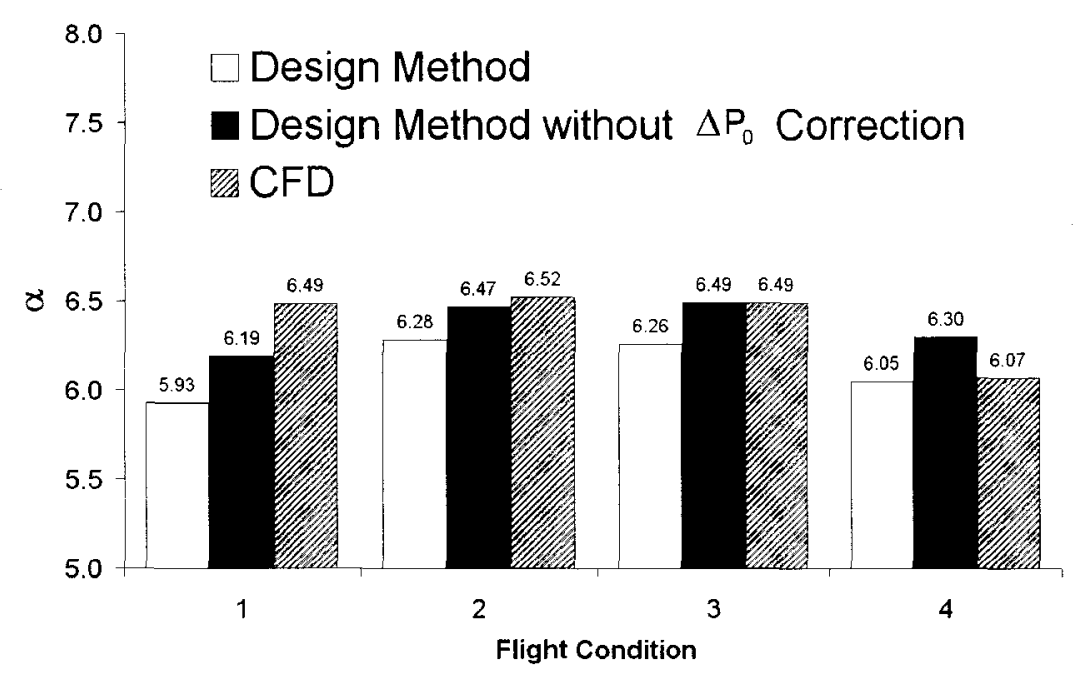

Figure 5.17: Comparison of CFD $\alpha$ to design method $\alpha$ with and without total pressure loss corrections.

\subsubsection{Mass Flow Rate}

The final comparison is made with respect to $\dot{m}$, which is the parameter of most interest in this air intake analysis. The percent difference of $\dot{m}$ calculated by the design method relative to the CFD simulations is shown in figure 5.18. At flight condition 1, which operates at a large flow capture ratio, the design method predicts flow separation along the cowl whereas the CFD results do not. Therefore the design method under predicts $\dot{m}$ by $8.6 \%$ at flight condition 1 . At flight conditions 2 and 3 , where there are no large regions of flow separation along the cowl or centre body surfaces, the design method under predicts $\dot{m}$ by about $3.6 \%-3.7 \%$ due in large part to the under prediction of $A_{3}^{(v i s c)}$. The design method results for these flight conditions is therefore a conservative estimate of the air intake $\dot{m}$ relative to the CFD results. At flight condition 4, where a large region of separated flow is present, the design method under predicts $\dot{m}$ by $0.3 \%$ due to an under prediction of $A_{3}^{(\text {visc })}$, and an under prediction of $P_{0_{3}}^{(v i s c)}$.

These results indicate that for flows where no flow separation is predicted, the design method will still tend to output conservative values of $\dot{m}$. At large flow capture ratios, the 
design method will tend to predict early flow separation along the cowl surface and thus under predict $\dot{m}$ due to an under prediction of $A_{3}^{(v i s c)}$. In cases where flow separation occurs along the centre body, the design method will tend to predict boundary layer separation downstream of the CFD separation point, but due to 3D affects the design method will predict a conservative value of $A_{3}^{(\text {visc })}$. However, an over prediction of $\rho_{3}^{(v i s c)}$, and $U_{z 3}^{(v i s c)}$ due to not accounting for total pressure losses after boundary layer separation results tends to counter the under prediction of $A_{3}^{(v i s c)}$ and therefore $\dot{m}$ is only slightly under predicted.

When the percent error plots for $A_{3}^{(v i s c)}, \Delta P_{0}$, and $\dot{m}$ are compared, it is clear that the error in $\dot{m}$ follows the behaviour of the $A_{3}^{(v i s c)}$ error. This result indicates that $A_{3}^{(\text {visc })}$, and thus the boundary layer results, is the dominant factor in the calculation of $\dot{m}$. As flight speed increases from flight condition $1-4$, the magnitude of the under prediction of $m$ decreases. Figure 5.19 shows that as flow capture ratio decreases, the magnitude of the under prediction of $\dot{m}$ decreases.

Recalling figure 4.3(a), which shows the design method to predict lower values of $\alpha$ than the rocket-ejector analysis, the design methods under prediction of $\dot{m}$ relative to the CFD simulations suggests that an RBCC engine using this particular air intake may operate at a higher $\alpha$ than predicted by the design method and thus have a higher thrust augmentation ratio.

Overall, for the air intake geometry and flight conditions used in this work, the design method under predicts $\dot{m}$ by up to $8.6 \%$ relative to 3D CFD. 


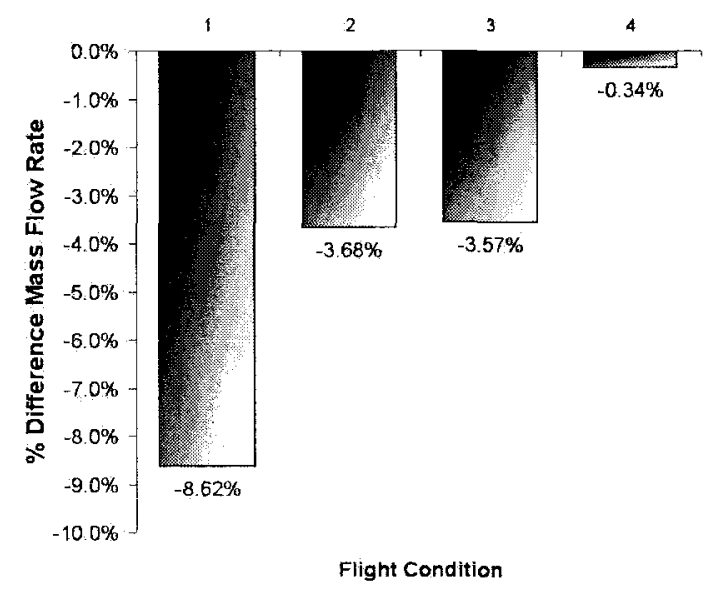

Figure 5.18: Percent difference of design method $\dot{m}$ from CFD $\dot{m}$ for different flight conditions.

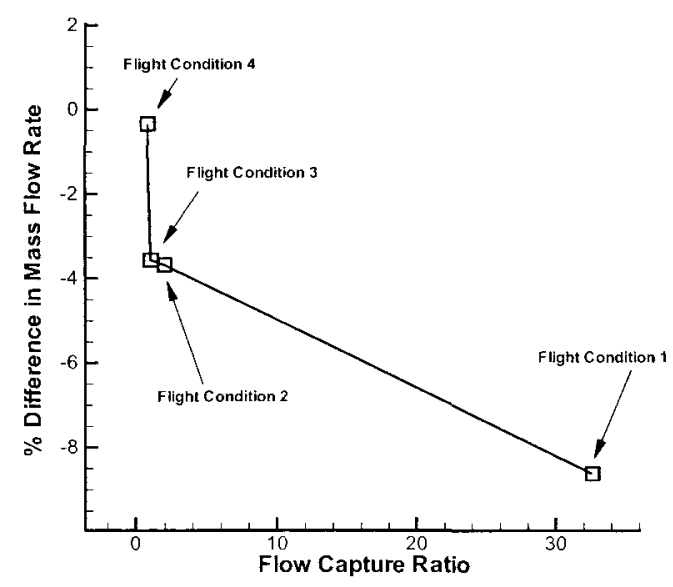

Figure 5.19: Percent difference of design method $\dot{m}$ from CFD $\dot{m}$ plotted against flow capture ratio. 


\section{Chapter 6}

\section{Conclusions and Recommendations}

\subsection{Conclusions}

A method for designing the air intake geometry for a novel RBCC rocket nozzle has been presented. The purpose of this design method is to completely define an air intake geometry for a novel RBCC rocket nozzle geometry given several user inputs, as well as to provide a rough performance estimate for the intake geometry. This design method consists of a geometry creation step in which a three-dimensional intake geometry is generated, and a simple flow analysis step which predicts the air intake mass flow rate and total pressure loss due to friction. Intake mass flow rate is used as a performance metric since it directly affects the amount of thrust a rocket-ejector can produce.

The air intake geometry is built using three types of bodies, an axisymmetric centre body, a cowl and nozzle fairings. The air intake design method defines these intake bodies using a given rocket nozzle geometry along with 8 primary geometry parameters. These 8 geometry parameters are $\sigma, \tilde{z}_{2}, A R, \tilde{r}_{C B_{1}}, C R, \tilde{t}_{3}, \tilde{c}_{\text {fair } r_{m a x}}$, and $A_{2-3}$. The geometry parameters $\tilde{z}_{2}$ and $A R$ control the length and inflow to outflow area ratio of the air intake while $C R$ controls the cowl lip geometry and $\tilde{r}_{C B_{1}}$ has a significant affect on the intake 
centre body shape. Variation of the geometry parameters is shown to allow for a large range of air intake shapes and can be used to optimize inlet performance.

The flow analysis used in the air intake design method combines an inviscid flow solver with a viscous flow solver. The inviscid flow field is computed using potential flow panel methods. Velocity scaling and compressibility corrections are also applied to the flow field to account for the presence of the fairing geometry and compressibility. The viscous flow solver then computes the boundary layer using an integral boundary layer method and the inviscid flow solution. Outflow plane properties such as the corrected flow area and total pressure loss are calculated using the viscous flow solution, which are then used to calculate intake mass flow rate.

The design method has been implemented in MATLAB ${ }^{\circledR} 7.0 .1$ and runs in approximately 2 minutes using a $2.4 \mathrm{GHz}$ processor. This short run time makes this design method practical for use with optimization programs.

A design method flow analysis and 3D CFD simulations have been conducted on an air intake geometry designed to fit around a novel RBCC rocket nozzle with the performance characteristics of an LE-7A rocket engine. Comparisons between the design method flow analysis results and 3D computational fluid dynamics simulations of this air intake operating at 4 different flight conditions along the Ariane 4 flight profile show the design method to under predict air mass flow rate by up to $8.6 \%$.

For fully attached flows it is found that as flight speed increases, the magnitude of the boundary layer thickness and viscous flow area error tends to decrease. When operating at high flow capture ratios, the design method is found to predict early flow separation along the cowl surface due to a predicted larger adverse pressure gradient.

In cases where boundary layer separation is present on the centre body surface, the design method tends to predict delayed boundary layer separation. However, due to 3D flow effects, and an under prediction of total pressure loss, air mass flow rate is still slightly 
under predicted.

The total pressure loss calculations through the air intake made by the design method tend to under predict the total pressure at plane 3 by up to $0.06 \%$ and over predict the total pressure by up to $0.15 \%$, with the highest error in flows with boundary layer separation.

The 3D CFD simulation results highlight flow situations where 3D effects become significant. At high flow capture ratios, a region of separated flow forms along the intersection between the cowl and fairing surfaces. At high flight speeds, the fairing geometry alters the pressure distribution along the centre body surface near the fairing, which alters the boundary layer development along the centre body. Both of these 3D flow features cannot be predicted by the design method.

For all four flight conditions tested, which represent points along the subsonic portion of an Ariane 4 flight profile, the design method analysis under predicts air mass flow rate by up to $8.6 \%$ relative to $3 \mathrm{D}$ CFD simulations. The design method mass flow rate error from the CFD simulations follows the same trends as the effective flow area error, indicating that effective flow area is a controlling factor in the air mass flow rate.

The results from the design method flow analysis and CFD simulations shows that the air intake design method tends to provide conservative estimates of air intake mass flow rate over a range of subsonic flight conditions, and for flows with or without regions of flow separation.

\subsection{Recommendations}

The current flow analysis method does not model flows with regions of flow separation accurately both in terms of corrected flow area and total pressure loss. Improvements to the corrected flow area calculation in flows with separation may be possible by coupling the inviscid and viscous flow solvers. While strong coupling may be impractical for this 
intake geometry, a weak-strong coupling method, where one boundary layer is held fixed and the other is coupled to the inviscid core, may be feasible.

Since the goal of a rocket-ejector configuration is to provide improved thrust over a pure rocket, an improved estimate of the rocket-ejector configurations thrust augmentation ratio could be achieved by estimating the drag due to the air intake geometry using the skin friction coefficient data.

The current centre body geometry is built using a series of hermite curves. While the slope of two adjacent curves are equal at their intersection point, their curvature is not necessarily the same. Discontinuities in curvature can result in undesirable pressure distributions. Therefore, a method for also matching curvature at the centre body region boundaries may be required.

Improvements to the current method used to generate the fillet geometry between the lip and fairing surfaces may help to prevent the formation of the separated flow region when the intake is operating at large flow capture ratios. Alternatively, blow in doors could be added to the cowl geometry to reduce the large peak velocities around the lip geometry and therefore prevent flow separation. The addition of blow in doors, open only at large flow capture ratios, to the panel method would require modification to the panel geometry organization and solution method, but is certainly possible. Blow in doors may also allow for the use of smaller lip $C R$ values, and thus a sharper cowl lip, which is preferred at higher flight speeds.

The compressibility correction method used in this design method currently treats the centre body and cowl surface velocities independently. The addition of a constant mass flow rate constraint through the duct portion of the intake may improve the accuracy of surface velocity calculations.

While the current flow analysis is limited to subsonic flight speeds, this rocket nozzle/air intake geometry is intended for use in $\mathrm{RBCC}$ vehicles which operate from the static 
condition up to hypersonic speeds. It would therefore be beneficial for the design method to predict the shock structures a particular intake geometry would likely see as the designer may wish to obtain a shock-on-lip condition for a particular flight condition.

Additional CFD simulations on different air intake geometries would help to determine how significant the 3D flow effects observed at high flow capture ratios and at high flight speeds become. It is suspected that as the number of nozzle clovers is increased, and therefore the flow blockage due to the fairing geometry increases, the 3D flow effects will become more significant.

Finally, the true accuracy of the air intake design method flow analysis can be evaluated by conducting physical experiments on a rocket nozzle/air intake configuration and comparing the experimental results for air mass flow rate and velocity and density values at plane 3 to the design method results. 


\section{References}

[1] Thorpe, D. G., "Economics of Launch Vehicles and Two Configurations for Tremendous Cost Reductions," AIAA 2009-5539, 2009, presented at the $45^{\text {th }}$ AIAA/ASME/SAE/ASEE Joint Propulsion Conference and Exhibit.

[2] "Fortune 500: Our annual ranking of America's largest corporations," 2009, http://money.cnn.com/magazines/fortune/fortune500/2009/snapshots/2255.html.

[3] Contant, C., "Economics of Space Transportation for the 21st Century," AIAA 2004-5918, Sept, 2004, presented at the Space 2004 Conference and Exhibit.

[4] Koelle, D., "Economics of Small Fully Reusable Launch Systems (SSTO vs. TSTO)," Acta Astronautica, Vol. 40, No. 2-8, 1997, pp. 535-544.

[5] DeBonis, J. R., Trefny, C., and Jr, C. S., "Inlet Development for a Rocket Based Combined Cycle, Single Stage to Orbit Vehicle Using Computational Fluid Dynamics," AIAA 99-2239, Jun, 1999, presented at the $35^{\text {th }}$ AIAA/ASME/SAE/ASEE Joint Propulsion Conference and Exhibit.

[6] Escher, W. J. D., The Synerjet Engine: Airbreathing/Rocket Combined-Cycle Propulsion for Tomorrow's Space Transports, Society of Automotive Engineers, Inc., 1997.

[7] Daines, R. and Segal, C., "Combined Rocket and Airbreathing Propulsion Systems for SpaceLaunch Applications," Journal of Propulsion and Power, Vol. 14, No. 5, 1998, pp. 605-612.

[8] Etele, J., Parent, B., and Sislian, J., "Analysis of Increased Compression Through Area Constriction on Ejector-Rocket Performance," Journal of Spacecraft and Rockets, Vol. 44, No. 2, 2007, pp. 355-364.

[9] Foster, R. W., Escher, J. D., and Robinson, J. W., "Studies of an Extensively Axisymmetric Rocket Based Combined Cycle (RBCC) Engine Powered SSTO Vehicle," AIAA 89-2294, Jul, 1989, presented at the $25^{t h}$ Joint Propulsion Conference.

[10] Congelliere, J., Prevatte, N., and Lime, J., "Advanced Ramjet Concepts, Volume II. Ejector Scramjet Technology," TR-67-118, The Marquardt Corp., Air Force Aero Propulsion Lab., May, 1968, Van Nuys, CA. 
[11] Dykstra, F., Caporicci, M., and Immich, H., "Experimental Investigation of the Thrust Enhancement Potential of Ejector Rockets," AIAA 97-2756, Jul, 1997.

[12] Escher, J. D. and Schnurstein, R. E., "A Retrospective on Early Cryogenic Primary Rocket Subsystem Designs as Integrated into Rocket-Based Combined-Cycle (RBCC) Engines," AIAA 93-1944, Jul, 1993, presented at the $29^{\text {th }}$ Joint Propulsion Conference.

[13] Nakane, M., Kobayashi, D., Yoshida, H., and Ishikawa, Y., "Feasibility Study on Singe Stage to Orbit Space Plane with RBCC Engine," $16^{\text {th }}$ AIAA/DLR/DGLR International Space Planes and Hypersonic Systems and Technologies Conference, 2009.

[14] Thomas, S. R., Palac, D. T., Trefny, C. J., and Roche, J. M., "Performance Evaluation of the NASA GTX RBCC Flowpath,” NASA TM 2001-210953.

[15] Siebenhaar, A., Bulman, M., Norris, R., and Thompson, M., "Development and Testing of the Aerojet Strutjet Combustor," AIAA 99-4868, 1999.

[16] Cerantola, D. J. and Etele, J., "A Nozzle Concept to Entrain Atmospheric Air for Ejector Operation," AIAA 2006-8089, 2006, presented at the $14^{\text {th }}$ AIAA/AHI Space Planes and Hypersonic Systems and Technologies Conference.

[17] Cerantola, D. J., Rocket Nozzle Design with Ejector Effect, Master's thesis, Carleton Univ., Mechanical and Aerospace Engineering Dept., Ottawa ON, 2007.

[18] Seddon, J. and Goldsmith, E. L., Intake Aerodynamics, $2^{n} d$ ed., AIAA, 1999, Reston, VA.

[19] Sovran, G. and Klomp, E. D., "Experimentally Determined Optimum Geometries for Rectilinear Diffusers with Rectangular, Conical or Annular Cross-Section," Fluid Mechanics of Internal Flow: Proceedings of the Symposium of the Fluid Mechanics of Internal Flow, 1965, pp. 270-319, Warren, MI.

[20] Childs, R. E., A Computational Method for Subsonic Compressible Flow in Diffusers, Ph.D. thesis, Stanford Univ., Dept. Mechanical Engineering, Stanford CA, 1981.

[21] Hess, J. L. and Smith, A. M. O., "Calculation of Potential Flow about Arbitrary Bodies," Progress in Aeronautical Sciences, Vol. 8, 1966.

[22] Wauquiez, C., Shape Optimization of Low Speed Airfoils using MATLAB and Automatic Differentiation, Ph.D. thesis, Royal Institute of Technology, Dept. Numerical Analysis and Computing Science, Stockholm Sweden, 2000.

[23] Hess, J. L., "Higher Order Numerical Solution of the Integral Equation for the TwoDimensional Neumann Problem," Computer Methods in Applied Mechanics and Engineering, Vol. 2, 1973, pp. 1-15.

[24] Hess, J. L., "Improved Solution for Potential Flow about Arbitrary Axisymmetric Bodies by the use of a Higher-Order Surface Source Method," Computer Methods in Applied Mechanics and Engineering, Vol. 5, 1975, pp. 297-308. 
[25] Hess, J. L. and Martin, R. P., "Improved Solution for Potential Flow about Arbitrary Axisymmetric Bodies by the use of a Higher-Order Surface Source Method: Part I. Theory and Results," NASA CR 134694, 1974.

[26] Moran, J., An Introduction to Theoretical and Computational Aerodynamics, Wiley, 1984, New York, NY.

[27] Lieblein, S. and Stockman, N. O., "Compressibility Correction for Internal Flow Solutions," Journal of Aircraft, Vol. 9, No. 4, 1972, pp. 312-313.

[28] Dietrich, D. A., Oehler, S. L., and Stockman, N. O., "Compressible Flow Analysis about Three-Dimensional Wing Surfaces Using a Combination Technique," $21^{\text {st }}$ AIAA Aerospace Sciences Meeting, 1983, Reno, NV.

[29] Harris, A., "Compressibility Corrections for Multifoil Sections," Journal of Aircraft, Vol. 23, No. 2, 1985, pp. 156-158.

[30] Peace, A. J., "Transonic Flow Calculations around Isolated Inlet Configurations," Aeronautical Journal, Vol. 90, 1986, pp. 103-110.

[31] Reddy, D. R., Reddy, E. S., and Moody, R. E., "Aerodynamic Shape Optimization of a Subsonic Inlet Using Three-Dimensional Euler Computation," Journal of Propulsion and Power, Vol. 14, No. 2, 1998, pp. 225-233.

[32] Cebeci, T., Shao, J. P., Kafyeke, F., and Laurendeau, E., Computational Fluid Dynamics for Engineers: From Panel to Navier-Stokes Methods with Computer Programs, Horizons Publishing, 2005, Long Beach, CA.

[33] Liou, M. S., Coakley, T. J., and Bergman, M. Y., "Numerical Simulation of Transonic Flows in Diffusers," MDRL 81-11, 1981.

[34] Head, M. R., "Entrainment in the Turbulent Boundary Layer," A.R.C., 1958.

[35] Green, J. E., Weeks, D. J., and Brooman, J. W. F., "Prediction of Turbulent Boundary Layers and Wakes in Compressible Flow by a Lag-Entrainment Method," R.A.E. TR 72231, 1973.

[36] Williams, J. C., "Incompressible Boundary-Layer Separation," Annual Review of Fluid Mechanics, Vol. 9, 1977, pp. 113-144.

[37] Johnston, J. P., "Diffuser Design and Performance Analysis by Unified Integral Methods," $33^{\text {rd }}$ AIAA/ASME/SAE/ASEE Joint Propulsion Conference and Exhibit, 1997, Seattle, WA.

[38] Yamashina, H., Fukushima, K., and Saijo, A., "CAD for Free-Form Surfaces," Computer Integrated Manufacturing Systems, Vol. 9, No. 1, 1996, pp. 9-18.

[39] Turner, M. J. L., Rocket and Space Propulsion: Principles, Practice, and New Development, Springer, 2005, New York, NY.

[40] "Earth Atmosphere Model - Metric Units," http://www.grc.nasa.gov/WWW/K12/airplane/atmosmet.html. 
[41] ANSYS ${ }^{\circledR}$,Inc., ANSYS ${ }^{\circledR}$ ICEM CFD/AI Environment, Release 11.0 Documentation, 2006.

[42] McFarland, E. R., "Solution of Plane Cascade Flow Using Improved Surface Singularity Methods," Journal of Engineering for Power, Vol. 104, Jul, 1982, pp. 668-674.

[43] Bradshaw, P., Ferriss, D. H., and Atwell, N. P., "Calculation of Boundary Layer Development Using the Turbulent Energy Equation," Journal of Fluid Mechanics, Vol. 28, No. 3, 1976, pp. 593-616.

[44] Dunham, J., "Prediction of Boundary Layer Transition on Turbomachinery Blades," AGARD Meeting on Boundary Layers in Turbomachines, 1972.

[45] Bardina, J., Lyrio, A., Kline, S. J., Ferziger, J. H., and Johnston, J. P., "A Prediction Method for Planar Diffuser Flows," Journal of Fluids Engineering, Vol. 103, Jun, 1981, pp. 315-321.

[46] Fox, R. W. and Kline, S. J., "Flow Regimes in Curved Subsonic Diffusers," Journal of Basic Engineering, Sept, 1962, pp. 303-313.

[47] "Encyclopedia Astronautica: LE-7A," http://www.astronautix.com/engines/le7a.htm.

[48] ANSYS ${ }^{\circledR}$, Inc., ANSYS ${ }^{\circledR}$ CFX, Release 11.0 Documentation, 2006.

[49] Grosvenor, A. D., Numerical Simulations of Diffusing S-Duct and Vortex-Generator-Jet Flows, Master's thesis, Carleton Univ., Mechanical and Aerospace Engineering Dept., Ottawa ON, 2000.

[50] Menter, F., "Influence of Freestream Values on $k-\omega$ Turbulence Model," AIAA Journal, Vol. 30, 1992, pp. 1657-1659.

[51] Bardina, J., Huang, P., and Coakley, R., "Turbulence Modeling Validation, Testing, and Development," NASA TM-1 10446, 1997.

[52] Reddy, D. R. and Sree, D., "Computational Study of Flow in a Rocket-Based Combined Cycle (RBCC) Engine Inlet," Computers and Fluids, Vol. 35, 2006, pp. 724-732.

[53] Freitas, C. J., Ghia, U., Celik, I., Roache, P., and Raad, P., “ASME's Quest to Quantify Numerical Uncertainty," AIAA paper 2003-627, Jan, 2003, presented at the $41^{s t}$ Aerospace Sciences Meeting and Exhibit. 